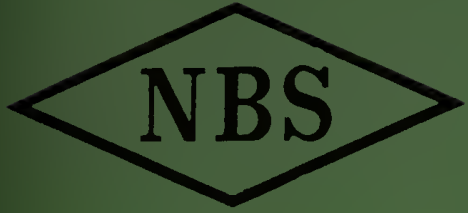

Eechnical Note

Boulder Laboratories

\title{
BIBLIOGRAPHY ON AURORAL RADIO WAVE PROPAGATION
}

BY

WILHELM NUPEN

U. S. DEPARTMENT OF COMMERCE NATIONAL BUREAU OF STANDARDS 


\section{THE NATIONAL BUREAU OF STANDARDS}

\section{Functions and Activities}

The functions of the National Bureau of Standards are set forth in the Act of Congress, March 3, 1901, as amended by Congress in Public Law 619, 1950. These include the development and maintenance of the national standards of measurement and the provision of me ans and methods for making measurements consistent with these standards; the determination of physical constants and properties of materials; the development of methods and instruments for testing materials, devices, and structures; advisory services to government agencies on scientific and technical problems; invention and development of devices to serve special needs of the Government; and the development of standard practices, codes, and specifications. The work includes basic and applied research, development, engineering, instrumentation, testing, evaluation, calibration services, and various consultation and information services. Research projects are also performed for other government agencies when the work relates to and supplements the basic program of the Bureau or when the Bureau's unique competence is required. The scope of activities is suggested by the listing of divisions and sections on the inside of the back cover.

\section{Publications}

The results of the Bureau's research are published either in the Bureau's own series of publications or in the journals of professional and scientific societies. The Bureau itself publishes three periodicals available from the Government Printing Office: The Journal of Research, published in four separate sections, presents complete scientific and technical papers; the Technical News Bulletin presents summary and preliminary reports on work in progress; and Basic Radio Propagation Predictions provides data for determining the best frequencies to use for radio communications throughout the world. There are also five series of nonperiodical publications: Monographs, Applied Mathematics Series, Handbooks, Miscellaneous Publications, and Technical Notes.

A complete listing of the Bureau's publications can be found in National Bureau of Standards Circular 460, Publications of the National Bureau of Standards, 1901 to June 1947 ( $\$ 1.25)$, and the Supplement to National Bureau of Standards Circular 460, July 1947 to June 1957 ( $\$ 1.50)$, and Miscellaneous Publication 240, July 1957 to June 1960 (Includes Titles of Papers Published in Outside Journals 1950 to 1959) (\$2.25); available from the Superintendent of Documents, Government Printing Office, Washington 25, D. C. 


\title{
NATIONAL BUREAU OF STANDARDS Eechnical Note
}

\author{
No. 128 \\ January 12, 1962
}

\author{
BIBLIOGRAPHY ON AURORAL RADIO \\ WAVE PROPAGATION
}

by

Wilhelm Nupen

\begin{abstract}
Prepared by
American Meteorological Society

Meteorological \& Geoastrophysical Abstracts
\end{abstract}

NBS Technical Notes are designed to supplement the Bureau's regular publications program. They provide a means for making available scientific data that are of transient or limited interest. Technical Notes may be listed or referred to in the open literature. They are for sale by the Office of Technical Services, U. S. Department of Commerce, Washington 25, D. C.

DISTRIB'JTED BY

UNITED STATES DEPARTMENT OF COMMERCE

OFFICE OF TECHNICAL SERVICES

WASHINGTON 25, D. C.

Price $\$ 2.75$ 



\section{TABLE OF CONTENTS}

Introduction $\ldots \ldots \ldots \ldots \ldots \ldots \ldots \ldots \ldots \ldots \ldots \ldots \ldots \ldots$

Subject Outline......................... iii

Geographical Outline ....................... xxiii

Chronological Index ..................... xxix

Bibliography on Auroral Radio Wave Propagation. . . . . . . . 1

Author Index. . . . . . . . . . . . . . . . . . . . . 105 

Note:

Abstractors: Bibliography on Auroral Radio Wave Propagation

MGA Staff Members:

A. A. . . . . . Andrew Assur

R. B....... Ronald Baker

C.E.P.B. .. C.E. P. Brooks

I. L. D. . . . Isadore L. Dordick

A. K....... A jmal H. Khan

A. J. M. . . . . Andrew J. Meglis

N. N. . . . . Nndem E. U. Nndem

W. N. . . . . Wilhelm Nupen

A. M.P. ... Alexis M. Poushkin

M. L. R. . . Mary L. Rice

M. R. .... Malcolm Rigby

I. S. ..... Ismail Saad

N.P.S. . . Nicholas P. Setchkin

E. Z. S... Evelyn Z. Sinha

O.T..... Otto Taborsky

G. T..... Geza Thuronyi

A. V. . . . A André V andenplas

\section{Others:}

L. A. M. . ......... L. A. Manning

S. F. . . . . . . . . S. Fritz

IRE, Proc........... Institute of Radio Engineers, Proceedings

Phys. Abstr........ Physics Abstracts

Sci. Abstr. . . . . . . . Science Abstracts

\section{Library Symbols:}

DBS. ...... U.S. National Bureau of Standards, Washington, D.C. DGS ...... U. S. Geological Survey, Washington, D.C.

DLC ...... Library of Congress, Washington, D.C.

DWB ....... U. S. Weather Bureau, Suitland, Maryland

DN-HO.... U.S. Navy Hydrographic Office, Suitland, Maryland 



\section{INTRODUCTION}

The present compilation is presented as the third of a series of bibliographies in preparation by Meteorological and Geoastrophysical Pabstracts for the Boulder Laboratories of the National Bureau of Standards.

Several such bibliographies, all on the general subject of electromagnetic (radio) wave propagation in the atmosphere or ionosphere, are being published in the NBS, Technical Notes Series. The first bibliography entitled "Bibliography on Ionospheric Propagation of Radio Waves (1923-1950)" was published in October 1960, as NBS, Technical Note, No. 84, and is available from United States Department of Commerce, Office of Technical Services, Washington 25, D. C. (PB 161585). Price \$7.00. It contains 1404 references, mostly annotated. The second: "Bibliography on Meteoric Radio Wave Propagation", containing 368 items, was published in May 1961 as NBSS Technical Note No. 94 (PB 161595). Price \$2. 75.

Similar bibliographies in preparation on "Radio Astronomy" and on "Tropospheric Propagation of Radio Waves" will be published in 1962.

The present compilation is made up of about 300 abstracts or titles from the literature published during the period 1893-1961. It is thus only a portion of the international literature on radio-auroral research. Omissions of pertinent papers are involuntary and it will be greatly appreciated if brought to our attention so they may be included in future supplements.

Auroral effects on radio waves Nere known before Oscanyan, in 1929 (C-222), actually observed the influence of visible aurora on radio communication. International research lagged until 1938, when Harang and Stoffregen discovered and identified auroral VHF scattering. Whereas at first the disturbances attending the aurora were considered only as a nuisance to radio communication, the auroral effects have now been exploited as a new and enhanced mode of communication and a tool for further exploration and research into the physical characteristics 
of the atmosphere.

For the most recent systematic discussion of the "Radio Aurora", the reader should consult Chapter 6 of the new (1961) book "Physics of the Aurora and Airglow", by J. W. Chamberlain of Yerkes Observatory. This excellent review of the subject was received after the present bibliography had been completed and indexed; hence it has been added as a supplementary item (C-297).

We wish to express our appreciation to Mrs. Evelyn Z. Sinha for her assistance in locating and editing much of the material in the files of $\mathrm{M} \& \mathrm{GI}$; to Mr. Otto Taborsky for preparing the geographical outline; to Miss. Muriel Haas for checking the accuracy of the bibliographic entries; and to Mrs. Doris Nickey for typing and correcting the manuscript. Finally, we wish to acknowledge the very considerable help rendered by various members of the staff of the National Bureau of Standards, Boulder Laboratories, especially Mr.. Bradford R. Bean, who has given us guidance and has coordinated our efforts with those of the Boulder Laboratories.

Additions and corrections should be addressed to:

Malcolm Rigby, editor

Meteorological \& Geoastrophysical Absts. P. O. Box 1736, Washington 13, D.C. 


\section{SUBJECT OUTLINE}

\section{GENERAL WORKS}

1. Textbooks, monographs, manuals. C-7, $32,40,59,126,145$, $172,200,202,234,241,255,259,275,277$, 283,297

2. General reviews and surveys. C- $6,28,49,76,88,117,159$, $160,165,173,247,275,276,297$

3. Data publications. C. $3,4,9,13,16,18,28,37,38,47,56,57$, $64,66,82,83,86,87,108,109,112,118,120$, $125,156,165,174,187,188,192,203,204$, $215,216,249,257,273,277$

a. Statistical data. C-66, 184, 187

b. Analyses. C-21, 22, 35, 38, 87

4. Theses. C-35, 82,260

5. Maps and nomograms. C-3, 4, 6, 22, 47, 215

6. Bibliographies. C.9, 11, 49, 64, 72, 105, 137, 143, 160, 163, $175,202,224,255,276$

7. History. C- $8,23,24,57,76,221,222,251-254,260,266-269$, 291

8. Conferences, symposia. C-38, 54, 64, 68, $111,149,171,177$

9. Research programs. C-5, 111, 226, 244

a. Expeditions. C-129, 221, 287

b. I. G.Y. C. $54,55,57,69,104,149,159,232,245,246,293$

10. Nomenclature. C-14,63, 76, 95, 108, 110, 126, 168, 171, 172, $179,209,257,288$ 
II. THEORIES
Alfven's C-7
Appleton's C.161
Birkeland's C.23, 24, 71
Birkeland and Störmer's C-7, 253
Booker's / scattering C-30, 84, 86
Booker, Gartlein and Nichol's C.84
Chamberlain's C.48
Chapman-Ferraro-Martyn's C-45, 86, 151, 247, 296
Harang and Landmark's C-84, 127, 174
Hulburt's C-71
Lindquist / Polar blackout C-172
Martyn's C-198
Meinel and Schulte C-174
Moore's / Trail analogy C-37, 84
Moore's C-207, 208
Peterson's C.149
Störmer's C-41, 71, 86, 215, 251-253
Vegard's C-71, 276
Miscellaneous
Aurora C.169
Auroral echo hypothesis C.164
Auroral mechanism C-16I
Auroral noise C.91
Electric field theory C.7
Experimental testing of theories C.52
Ionosphere C.72
List of German theories C.137
Modes of reflection C-174
Molecular diffusion C-31
Plane earth C-16
Review of current theories C-49, 275, 297

III. FORMULAS
Auroral radar C-166
Determination / propagation constant C.13
Radar C.166
Small scale eddies C-3I 
IV. MODEIS

1. Theoretical

Atmosphere C-259

Atmosphere, upper C.209

Atmosphere, underdense ionosphere C-233

Auroral ionization C.203

Protons C.259

SC magnetic storms and aurora C-247

Other C-112, 185

2. Experimental (laboratory) models

Bennet's C.296

Birkeland's C.23, 24, 253

Brüche's C.41

Malmfors' C-7

Torus space C.71

V. ATMOSPHERIC AND COSMIC STRUCTURE AND PHYSICS

1. Atmospheric Stratification

a. Troposphere C-31

Arctic C-9

Thunderstorms C-138

b. Ionosphere C-158

Ionospheric disturbances C-9, 11, 18, 21, 31, 95, 121, $155,168,196,213,265$

Large eddies C.31

Small eddies C.31

Elongated irregularities C-213

Ionized columns C. $28,30,38,174,207,242$, 271

Ionized columns diam. C-208

Spiral patterns C-121

Arctic disturbances C-33

Measurements C-19, 27

Currents C-39

SI D C.65

C. Upper atmosphere C-193, 194

Electric currents C-24 
2. Layers

a. D-region C-11, 78, 131, 162, 223, 228, 238

Damping effect C-162

Disturbed periods C-16

b. E-region C-18, 22, 30, 60, 74, 75, 79, 94, 182, 211, 216, $218,223,228,235,256,278,291$

$\mathrm{E}_{\mathrm{S}} \quad \mathrm{C}-75,79,83,85,119,127,136,139,140,156,194$, $197,200,219,229,231,241,285$

Diumal variation C.83, 173

Seasonal variation C-173

Classification C-227

E2 $\quad$ S -79

Spiral occurrence C.79

$N_{1} \quad \mathrm{C}-136$

Gloving $\mathrm{E} \quad \mathrm{C}-13$

c. F-region C-18, 60, 74, 107, 119, 140, 210, 229, 245, 278

Irregularities C.16, 18, 72, 131, 196, 223

Irregularities - height C-16

Overhead changes C-18

Tilt C.248

$F_{1}$ reflections C-18, 79,286

$F_{2} \quad C-22,74,79,194,195,216,288$

Reflections C-18,22, 227

d. Other
Absorbing layers C-102
Below E C.27
Structure C-225, 237
Auroral C-18, 46, 141
Layer height C-46, 124, 139, 140, 197
Inbreak layers C-168
Horizontal layers C-116, 270

3. Clouds

Cirrus C-24

Ionization clouds C-103, 119, 151, 258

Velocity C-119

Luminescent clouds C-6I, 176 
4. Winds C-112, 211

Norwegian anticyclones

Vertical air movement C-124

5. Outer atmosphere C.92

Exosphere, fluctuations C.118

6. Miscellaneous

Temperature measurements C-65, 78

Plasma oscillations C.95

Air glow C-54, 193

Night air glow C-14

Atmospheric electricity C.9, 146

7. Stellar bodies

a. Meteors C-10, 168

Trails C-3I

Influences C-72, 84, 228, 231, 291

b. Radiostars

Scintillation C. $30,31,40,149,175,280$

c. Moon C-95, 147

Moonlight C.12

d. Sun

Solar activity C- $9,21,60,94,142,157,168,249$, $257,273,288,291,294$

Activity variation C.82

Solar flares C-5, 11, 133, 168, 235, 281, 289

Solar sunspots C-5, 93, 45, 50, 146, 223

Solar sunspot maximum C.5, 6

Solar streamers C-51,60, 196

Density C.51

UV light C-56

Solar corpuscles C-58, 60, 117, 121, 172, 194, 247

Equinoxes C-19, 28

Mögel-Dellinger effects C-168 


\section{viii}

\section{AURORAL PHENOMENA}

1. Types

$$
\begin{aligned}
& \text { Artificial C-14, 23, } 24 \\
& \text { Australis C-53, 57, 129, } 242 \\
& \text { Borealis C-10,13, 34, 37, 38, 53, 61, 94, 129, 134, 176, } \\
& 231,261,274 \\
& 222,223,230,243,265,281,282 \text {, } \\
& 234
\end{aligned}
$$

Subvisual C-80

Invisible C.200

Low frequency type C.149

Overhead C. $65,174,208,287$

Luminous C-58, 115

Red C-126, 133, 236, 294

"Radio aurora" C.63, 297

2. Forms

Homogenous C-38

Rayed C.38, 67, 101, 109, 179

Pulsating C.139, 140, 190

Nonpulsating C-139

Daytime form C-166, 233

Night time form C-166, 233

Curtain form C.138, 161

Curl form C-179

Diffuse C-174

Flaming C-190

Bright displays C.95, 156

3. Classification C-181

Generation C-10, 12, 14, 56, 97, 161, 175, 276

4. Aurora structure

Spectra C.14, 117, 277

Monochromatic arc C-14

Reflecting light C-73

a. Streamers C.61, 138, 176, 219

Rayed structure C-67, 179

Rays C. $48,53,258$

Rays, diameters C-48 
Curtains C-161

Curls C-179

Atomic rays $\mathrm{C}-14$

Cosmic rays C-11, 146, 289

Hydrogen rays C-14

Oxygen rays C-14,58

Red rays C-80, 91

Nitrogen rays $\mathrm{C}-14$

$\mathrm{X}$ - rays C.56

Nitrogen bands C.218

Calcium C.1I

b. Arcs C-138, 141, 154, 179, 199

c. Rings C.71, 296

d. Particles C-90, 110, 111, 164

Protons C.1, 48, 56, 58, 112, 259

Proton gyrofrequency C.118

$1 \mathrm{Mev}$ protons C.207

$\alpha$ photons C-58

Atomic ions C-11

Molecular diffusion C-3I

Nitric oxide molecules C.56

Particle mass C-110

Particle flux C-133

Particle bombardment C.285

Particle radiation $\mathrm{C}-288$

Particle precipitation

Precipitation, spiral C-120, 121, 161

Particle interaction C-110, 112, 113

5. Auroral aspects

Auroral activity C.9, 11

Auroral periodicity C-10

Diurnal variation C.114

Auroral aspect classification C.63

Aspect sensitivity C-39, 103, 233, 271

Auroral movements C.10, 43

Auroral movement speed C.43

Movements, horizontal C.154

Auroral depth range C.26, 46 
Auroral corona discharges C.138

Brush discharges C-138

Electric discharges C-46, 48

Auroral luminosity C-58, 110, 139, 192, 212, 218, 232, 289

Auroral display intensity C.50, 97, 197

Auroral interaction C-110, 112, 113

Auroral noise C-50

Auroral audibility C-138

Auroral radiation $\mathrm{C}-209$

Auroral mechänism C-86, 117, 161, 277

6. Miscellaneous

Laboratory experiments C-7, 23, 24, 41,64, 71, 111, 117

Spectrograms C-118

7. Auroral zones, regions, layers

a. Zones C-78, 80, 84, 89, 104, 118, 154, 164, 189, 194, 246, $257,286,290$

Absorption zone C.2, 11

Attenuation C-5

Micropulsations C-I

b. Regions C-58, 119, 149

Subauroral regions C-149

Minauroral regions C-210

c. Layers C-18, 46, 141

Layer heights C-46, 124, 139, 140, 197

Inbreak layers C-168

VII. PHYSICO-CHEMICAL FACTORS AND PROCESSES

1. Magnetic activities C- $3,6,9,23,82,88,91,108,109,115$, 211,213

Periodicity C-56, 95

Magnetic field state C-79, 213

Magnetic field lines C-17, 25, 53, 247, 279

Magnetic pole C-157, 159

Magnetic equator C-39

Magnetic micropulsations C-1

Magnetic ground currents C.239

Magnetic light C-198

Magnetic relationships C-114, 139, 159 
2. Magnetic storms and disturbances

Storms C-7, 86, 93, 122, 125-127, 142, 180, 181, 200,

$218,259,280,288$

Disturbances C-11, 17, 20, 21, 33, 65, 80, 92, 95, 118, $121-123,151,168,173,189,198$, $199,212,215,232,235,236,244$, $258,266-269,278,279,281,282$, 284,294

SC C-121, 180

Bays C-60, 140, 151, 194, 247, 285, 289

Positive C-198

Negative C-198

Miscellaneous

Earth's declination C-I3I

Earth's lines of force C-150, 152, 174

Earth's rotation C.8, 154

3. Ionization C-16, 30, 45, 62, 68, 102, 104, 133, 139, 140, 141, $149,151,159,166,207,229,278$, 291

Density C.22, 61, 212

Distribution C-148, 188, 196

Height of maximum ionization C-139, 219

Photoionization C.19

Drifts C-20,43, 45, 148, 151, 196, 211, 212

Low level ionization C-235

Ionizing agents C-I72

X-rays C-56

Clouds C-103, 119, 151, 258

Cloud velocity C.I19

Columns C-207, 271

Columns diameter C-208

Horizontal layers C-116, 270

Mechanism C.56, 171, 203, 204, 218, 219

Maintenance C.259

Ionograms C.210 
4. Electron characteristics

Density

per $\mathrm{cm}^{3}$

$4 \times 10^{4} \quad$ C. 101

$>4 \times 10^{4} \quad$ C. 141

$10^{5}$

C.51

$5 \times 10^{5}$

C. 211

$6 \times 10^{5}$

C.10

$10^{6}$

C. $-48,51$

$<2.6 \times 10^{7} \mathrm{C}-141$

$2.6 \times 10^{7} \quad$ C.295

$4 \times 10^{7}$ C.67

$6.5 \times 10^{7} \quad$ C.295

$10^{8}$

C. 48,100

$1.4 \times 10^{8} \quad \mathrm{C}-67,218$

$10^{11}$

C.98 per $\mathrm{m}^{3}$

$3 \times 10^{13}$

C.204

$6 \times 10^{12}$

C. 48

$\operatorname{per} m^{2}$

$10^{-17}$

C-147

Density, general C-30,40,48, 100, 107, 126, 161, 207, 211, $232,235,274$

Maximum height C-140

Electron energies C-14,58

Gyro power C-13, 67

Penetration C-58

Radiation C-41

Flux C-48

Flux, primary C.58

Flux, secondary C.58

Speed C.211

Collision frequency

Fast electrons C-14 


\section{xiii}

5. Reflection C. $21,43,45,51,53,61,67,70,73,76,82,86,101$, $107,123,199,240,256$

Characteristics C-22, 107, 192, 204

Mechanism C.102, 164, 182, 185, 219, 242, 278

Diumal variation C. 28,82

Seasonal variation C-28, 82

Oblique C-11, 16, 17, 89, 107, 114

Ground C-28

$F_{1} \quad C-18,79,286$

$\mathrm{F}_{2} \mathrm{C} \cdot 18,22,227$

"Reflecting wall" C-76

6. Reflection distances

$\perp 100 \mathrm{~km}$

$8.20 \mathrm{~km} \quad$ C. 125,126

$17 \mathrm{~km} \quad$ C- -125

$32 \mathrm{~km} \quad \mathrm{C}-58$

$50 \mathrm{~km} \quad$ C.56

$75.90 \mathrm{~km} \quad$ C. 256

$80 \mathrm{~km}$ C.58, 182, 219,

$60.100 \mathrm{~km} \quad$ C. 125

$93 \mathrm{~km} \quad$ C. 243

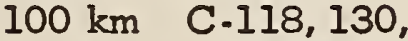
213,219

$>100 \mathrm{~km} \mathrm{C.172}$

$\ngtr 500 \mathrm{~km}$

$80.300 \mathrm{~km} \quad$ C. 213

$100.300 \mathrm{~km} \quad$ C.10

200.400 " C. 126

300 " C. 240

$350.500 "$ C.16

$>1,000 \mathrm{~km}$

$300.1,200 \mathrm{~km} \mathrm{C.248}$

500-1, 100 " C.84

$500.2,500$ " C.126

$600.1,100 "$ C.101

1,400 " C.260

$1,400-4,700 \mathrm{~km} \quad$ C. 164
$\$ 200 \mathrm{~km}$

$90.160 \mathrm{~km} \quad$ C. 106

$100.120 \mathrm{~km} \quad$ C. 128

$110 \mathrm{~km} \quad$ C. $46,270,279$

$110-120 \mathrm{~km} \quad \mathrm{C} .272,273$

116.165 " C.243

120 " C-136, 232

125 " C-258

$<125$ " C.43

150.200 "C.126

200 "C-141, 258 $\phi_{1,000 \mathrm{~km}}$

$80.1,000 \mathrm{~km} \quad$ C. 117

400.730 "C. 128

400.800 " C. 125,126

$500-800 "$ C.126

$500-900 "$ " 184

600 " C.174

900 " C.284

480 miles C.6I 


\section{xiv}

7. Absorption C.58, 88, 96, 139, 140, 155, 171, 175, 177, 190, 191, $197,215,223,235,238,285,286,289$

Nighttime C-11, 155

Daytime C-11, 56

Nondeviate C-56

Height C.219

8. Radiation C- $60,78,118,209,250$

Cosmic C-11, 96, 146, 289

Soft C-56

Van Allen belts C.88

Particle radiation $\mathrm{C}-19,78,79,146,288$

Photon C-218

Electron C-41

Lyman $\propto$ C.56, 58

Zones C.215

Maps C-215

Wave radiation $\quad$ - -79

U V C.78, 146, 159

X C $-6,14,19,56,194,289$

Thermal radiation $\mathrm{C}-78,250$

Miscellaneous C.209

From empty s'ky C.65

Cerenkov radio emission C.90

\section{RADIO COMMUNICATION}

1. Propagation

Forward scattering C.2, 11, 29, 31, 235

Anomalous ionospheric propagation C-34

"Super propagation" C-257

Propagation constant C-13

Miscellaneous

Slrip effect C-205 
2. Frequencies

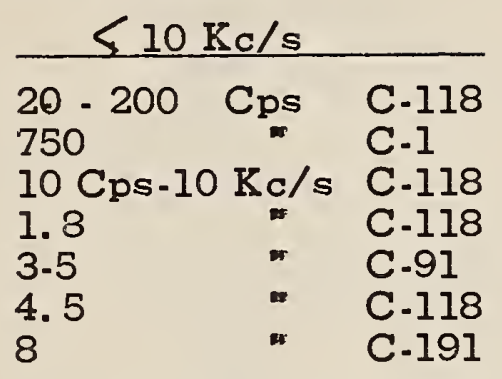

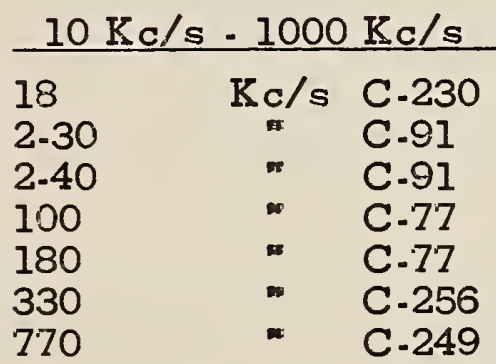

\begin{tabular}{|c|c|c|}
\hline $\begin{array}{l}2 \\
3 \\
4.6 \\
5.6 \\
6 \\
6.42 \\
3.8 \\
8 \\
3.9 \\
9.44 \\
10\end{array}$ & $\begin{array}{c}\mathrm{Mc} / \mathrm{s} \\
" \\
" \\
" \\
" \\
" \\
" \\
"\end{array}$ & $\begin{array}{l}\text { C- } 78 \\
\text { C- } 258 \\
\text { C- } 80 \\
\text { C- } 79 \\
\text { C- } 195 \\
\text { C. } 229 \\
\text { C- } 123 \\
\text { C-5 } \\
\text { C. } 126 \\
\text { C. } 25 \\
\text { C. } 5,21\end{array}$ \\
\hline
\end{tabular}

$10 \mathrm{Mc} / \mathrm{s} \cdot 20 \mathrm{Mc} / \mathrm{s}$

$\begin{array}{lll}11 & \mathrm{Mc} / \mathrm{s} & \mathrm{C}-5 \\ 12 & * & \mathrm{C}-174,223 \\ 12.86 & * & \mathrm{C}-229 \\ 7.13 & * & \mathrm{C}-126 \\ 14 & * & \mathrm{C}-292 \\ 15 & * & \mathrm{C}-5 \\ 1.16 & * & \mathrm{C}-172 \\ 2.16 & * & \mathrm{C}-195 \\ 16.7 & * & \mathrm{C}-260 \\ 19 & * & \mathrm{C}-260 \\ 17.31 & * & \mathrm{C}-70,229 \\ 18 & * & \mathrm{C}-96 \\ 1.20 & * & \mathrm{C}-219 \\ 20 & * & C .225\end{array}$

\begin{tabular}{lcl}
\multicolumn{3}{c}{$20 \mathrm{Mc} / \mathrm{s}-30 \mathrm{Mc} / \mathrm{s}$} \\
\hline 1.25 & $\mathrm{Mc} / \mathrm{s}$ & $\mathrm{C} .17$ \\
25 & $"$ & $\mathrm{C}-174$ \\
25.4 & $*$ & $\mathrm{C} .36$ \\
28 & $\cdots$ & $\mathrm{C} .264$ \\
15.30 & $"$ & $\mathrm{C} .222$ \\
30 & $"$ & $\mathrm{C} .3,235$
\end{tabular}

$30 \mathrm{Mc} / \mathrm{s}-40 \mathrm{Mc} / \mathrm{s}$

$\begin{array}{lll}32 & \mathrm{Mc} / \mathrm{s} & \mathrm{C} .85,132 \\ 33 & & \mathrm{C}-182,228 \\ 35 & & \mathrm{C}-127 \\ 36 & & \mathrm{C}-116\end{array}$

$40 \mathrm{Mc} / \mathrm{s} \cdot 50 \mathrm{Mc} / \mathrm{s}$

$\begin{array}{lll}41 & \mathrm{Mc} / \mathrm{s} & \mathrm{C} .17,170 \\ 41.15 & \mathrm{C}-211 \\ 43 & \mathrm{C}-95 \\ 44.10 & \mathrm{C} .95 \\ 46 & \mathrm{C}-61,295 \\ 48 & \mathrm{C}-188 \\ 48.2 & \mathrm{C} .178,179 \\ 48.5 & \mathrm{C}-187 \\ 30.50 & \mathrm{C}-62,204 \\ 32.50 & \mathrm{C}-102 \\ 43.50 & \mathrm{C}-34,81,82,83, \\ 50 & & 113,174,186, \\ & & 255\end{array}$

$50 \mathrm{Mc} / \mathrm{s}-100 \mathrm{Mc} / \mathrm{s}$

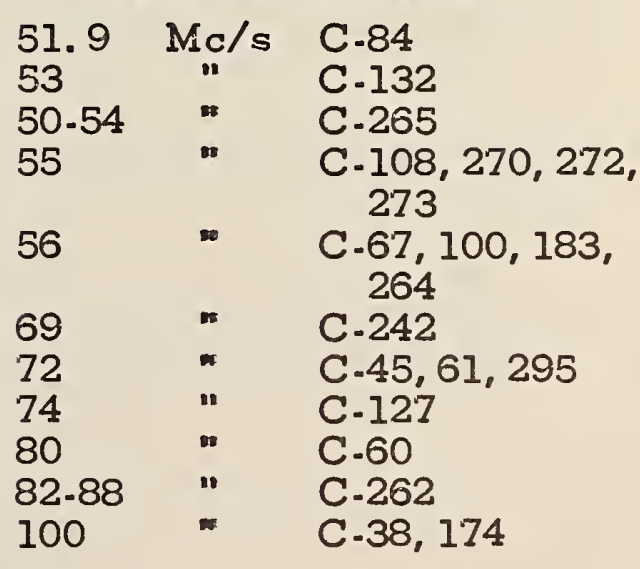


$100 \mathrm{Mc} / \mathrm{s} \cdot 150 \mathrm{Mc} / \mathrm{s}$

$106 \mathrm{Mc} / \mathrm{s}$ C.37, 67, 174, 183,211

106. 1 C. 240

$106.5 *$ C. $99,100,101$

$112 m$ C. 264

140 " C -85

28-148" C-205

$25.150 "$ C -38
$150 \mathrm{Mc} / \mathrm{s} \cdot 500 \mathrm{Mc} / \mathrm{s}$

$\begin{array}{lll}210 & \mathrm{Mc} / \mathrm{s} & \mathrm{C}-166 \\ 398 & * & \mathrm{C}-166 \\ 400 & \because & \mathrm{C}-167 \\ 488 & * & \mathrm{C}-25,26,166 \\ 500 & * & \mathrm{C}-133\end{array}$

$500 \mathrm{Mc} / \mathrm{s}-3000 \mathrm{Mc} / \mathrm{s}$

$1,306 \mathrm{Mc} / \mathrm{s} \quad \mathrm{C} .86$

2,800 " C.65

$3,000 \quad$ " C-67, 97, 99

b. Other frequencies

Audio frequency bands C.1, 118, 127, 205, 205

LF C $-90,91,118,230,256$

HF C. $5,88,199,227,260,290$

VHF C.11, 17, 29, 37, 38, 81-83, 132, 155, 170, 175, 188,

UHF C-25, 94, 147, 175, 233

$199,200,205,211,227,233,235,271$

Choice of frequency C.72

3. Communication links

Telephone C.15

Telegraph C.15

Short wave C.122, 217, 244

Amateur communication C.34, 165, 264

FM C.95

TV C.174, 262

Networks C.63,69, 245

Communication forecasting C.94, 165, 217

Communication geometry C.165

Communication enhanced C-237

4. Communication conditions

a. Transmission

Over auroral paths C.5, 17

VHF scattered transmissions C-29

AM speech transmissions C. 34

Experimental Transmissions C-82, 174, 201, 223, 237, 257 
b. Reception

TV C-174, 262

FM C.95

5. Communication disturbances

Polar blackouts C-3, 4, 6, 66, 121, 158, 171-173, 194, 285 287,290

Types C.6

Latitude variation C.6

Contour plots C. 3,4

Magnetic disturbances C- $7,11,17,20,21,33,65,80,86$, $92,93,95,118,121-123,125-127$, $142,151,168,173,180,181,189$, $199,200,212,215,218,232,235$, $236,244,258,259,266-269,278-$ $282,284,288,294$

Topographical C.221, 222

Blocking C-15, 115, 142, 221, 236, 265, 292, 294

Noise C.60, 77, 80, 88, 90, 91, 96, 132, 175, 228, 242, 289

Cosmic noise C-11, 77, 235

Energy C-118

Signal origin C.118

Chorus C-209, 228

Sferics C.9, 162, 221, 222

Whistlers C.87, 207

Hiss C-1, 77, 138, 191

Flutters C.292

Clicks C-138

Grinders C.138

Miscellaneous effects C.15, $71,122,135,180,181,222$, 230, 266-269

On TV channels C.262

Nuclear explosions C-168

Ice masses C.244

Mögel-Dellinger effect C-168

Faraday effect C-175

Doppler effect C.26, 28, 36, 38, 39, 95, 112, 167, 
6. Paths

Distances

$\begin{array}{lll}125 & \mathrm{Km} & \mathrm{C}-93 \\ 250 & " & \mathrm{C}-243 \\ 480 & " & \mathrm{C}-295 \\ 325-500 & \text { " } & \mathrm{C}-243 \\ 800 & \text { " } & \mathrm{C}-84 \\ 860 & \text { " } & \mathrm{C}-102 \\ 1,000 & \text { " } & \mathrm{C}-29 \\ 2,000 & \text { " } & \mathrm{C}-29 \\ 5,200 & \text { " } & \mathrm{C}-220 \\ 15,000 & \text { " } & \mathrm{C}-244,286 \\ 25,000 & \text { " } & \mathrm{C}-244\end{array}$

Miscellaneous

Secondary paths C-33

Great circle paths C-153, 257

Dark hemisphere paths C.11,60,151

IX. WAVE CHARACTERISTICS

1. Scattering C-28, 29, 116, 174, 126, 200, 207

Sources C-17, 102, 210

Mechanism C.63, 200, 203, 204

Ground C-16, 223

Back scatter C-31, 127, 200, 213, 223

Forward scatter C-31, 235

2. Waves

US W C-125, 126, 160

Short C-160, 274, 286

Cosmic radio waves C.96

Gyro waves C.13

Shock waves C-13

Radio wavelengths

$\begin{array}{ll}10 \mathrm{~cm} & \mathrm{C}-50,98 \\ 4 \mathrm{~m} & \mathrm{C}-279 \\ 7.3 " & \mathrm{C}-125 \\ 8 " & \mathrm{C}-73,279 \\ 10 " & \mathrm{C}-33,136,200 \\ 23 " & \mathrm{C}-86 \\ 37.08 \mathrm{~m} & \mathrm{C}-257 \\ 50 \mathrm{~m} & \mathrm{C}-221 \\ 84 " & \mathrm{C}-18 \\ 86 " & \mathrm{C}-278\end{array}$


xix

X. ECHO CHARACTERISTICS

1. Echo types

Delayed echoes C. 8,42

Diffuse echoes C-46, 134, 166, 229, 233

Discrete echoes C-17, 134, 166, 233

$E$ echoes C-123, 126

Slant $E_{s}$ echoes C-17, 19, 156, 168, 173

$F$ echoes C.123, 126

$\mathrm{F}_{1}$ echoes $\mathrm{C}-16$

"Life-timed" echoes C-126

Meteor echoes C-175, 182

Moon echoes C-147, 175

Radar echoes C-178, 179, 192, 199, 208, 211, 212, 219

Radio echoes C.183, 219

Scatter echoes C-182, 183

SW echoes C-274

UHF echoes C.81

World wide echoes C-76, 260

2. Echo analysis C-35, 38-40,43, 119, 127, 164, 176, 187, $188,200,273$

Echo geometry C-47, 53, 103, 129, 229, 271, 272, 279

Echo directions C-84, 167, 177, 178, 270, 272

Echo polarization C-34, 123, 147, 152, 184, 205, 270

Echo spectra C-167, 185, 207, 208

Echo amplitude C-11,81, 100, 118, 127

Echo distribution C.45, 103, 1.05, 160

Echo ranges C.128, 211, 295

Echo angle of arrival C-8, 25, 110, 128

3. Echo fading C- $30,39,110,122,126,172,241,278,288,290$

4. Miscellaneous

Echo absence C.99

"No echo periods" C-288 
XI. METHODS and TECHNIQUES of OBSERVATION

1. Radar methods C-21, 22, 25, 26, 38, 62, 69, 84, 97, 98, 101, $108,134,136,188,233$

Simultaneous radar - photographic C-38

2. Radio methods C-10, 14, 129, 193, 203

Experimental transmissions C-38

Cross modulation C-171

Spaced transmitter-receiver technique C-25, 26, 63, 92, $123,126,147,225$

Monitoring techniques C-18, 37, 100, 101, 127, 147, 225 Soundings C. $83,107,158,245$

Echo method C-72

3. Various techniques

Drifting station (SP-3) observations C-79

Rocket methods C-14,58, 193

Satellite methods C-225

Ship observations C-180

Optical observations C.36, 201

Photographic observations C-97, 101, 144

Tape recording C-38

C W - techniques C-81, 167, 171

Pulse method C-8I

Interference method C-128

Spectrographic observations C-69

Method for separating Es and auroral ionization C.83

Method for obtaining sudden absorption of cosmic noise C.96

Method for auroral detection C-14

Method for probability of occurrence of polar blackout C. 4,6

Method for change of frequency C.72

Method for utilizing scaled data to obtain echo activity index C.170

Visual method of influence of atmospheric conditions on radio reception $\mathrm{C}-201$

XII. INSTRUMENTS and EQUIPMENT

Antennas

\author{
Multivibratory C-22 \\ Semivibratory C-22 \\ Parabolic C-22 \\ Directional C-29, 81, 97, 127
}


Antennas (cont'd.)

Rotary C.89, 136

Continuously rotating C.84

Receiving C-34

Transmitting C-34

YAGI C-127

Half wave C-12

Automatic equipment C-195

Balloon borne instruments C-289

Cameras C-22, 144

Experimental equipment C-291

Experimental separation system of ground wave and sky

Fading rate counters C-122 waves C-201

Ionospheric recorders C-256

Ionosondes C.210

Low power radio equipment C-281

Low sensitivity equipment C-279

Magnetometers C.132

Oscillographs C-22

Panoramic recorders C. 172

Photomultipliers C-132

Radars C.21, 25, 101, 106

Bistatic type C.25, 106

Equipment C-26, 62

S C R 270 C.37

$50 \mathrm{Mc} \quad \mathrm{C}-186$

Continuous recording automatic radars C-186

VHF radars C. 170,188

$400 \mathrm{Mc} \quad \mathrm{C} .167$

Double Doppler C-185

L-band C-86

Low power FM radars C.62

High power pulse systems C-62

Ship borne radars C.85

$\mathrm{I}$ band radars $\mathrm{C}-86$

Mc Namara's radar system C.283

Rockets C.14

Satellites

Explorer VI C-88

Sounding equipment C-19

Spectrographs C-69

Spectrometers C.69 


\section{xxii}

Miscellaneous

Description of instruments C-126, 129, 134, 152, 170,

$218,222,232,241$

Modulation system design C-29

Design considerations / radars C-291 


\section{GEOGRAPHICAL OUTLINE}

\section{A. HEMISPHERES AND REGIONS}

1. Northern Hemisphere C.3, 53, 74, 95, 215, 273

2. Southern Hemisphere C.74, 215

3. Western Hemisphere C.69

4. Polar regions C. $27,33,79,119,157,158,285$

Arctic C.16, 33, 174, 215, 221, 222, 227, 245

North Pole C.79, 244, 245

Franz Joseph Land

Tikhaia Bay C-9

Svalbard

Spitsbergen C-245, 293

Longyearbyen C-216

North-east Land C.287

Björnöya C-216, 293

Antarctica C.245

Byrd Station C.191

Halley Bay C.19, 129, 130

Mirny C.244

Pioneerskaya C-244

Port Lockroy C.131

Port Stanley C.13I

South Pole C.244

5. Temperate regions C-78

B. CONTINENTS

1. Africa

South Africa C-265, 292 
2. Asia

India C-57

Japan C-33, 135, 210

Hiraiso Radio Wave Observatory C-122

Kokubunji C-210

Tokyo C-257

Lebanon

Beirut C-135

Pakistan C-57

U.S. S. R. in Asia

Cape Schmidt C.22

Dickson Island C-22

Siberia C-33

Tiksi C-22

Yakutsk C.22

3. Australasia C-243

Australia

New South Wales

Camden C-80, 82

South Australia

Adelaide C-92, 284

Tasmania C.77

Macquarie Island C-163, 190

New Zealand C-33, 288

Bluff C-270

Campbell Island C-243

Christchurch C-243, 288

Invercargill C-108, 109, 272, 273

South Island C.288

4. Europe C-260, 265

Austria C-236

British Isles C-15, 189

England C-126

Abinger C-292

Jodrell Bank C-43, 44, 47, 60, 116, 129, 134, 148, 
Scotland

Eskdalemuir C-45, 282

Lerwick C-282

Rosshire

Fortrose C.2S2

Denmark C-238

France

Dijon C-33

Haute Provence, Observatory of C-14

Paris C-135, 257

Germany C-177, 236, 239

Beelitz C-278

Berlin C-239

Bonn C.142

Göttingen C-142

Leipzig

Collm Observatory C-115

Scharmützelsee

Pieskow C-278

Greece C.236

Netherlands C.177

Eindhoven C.251

Norway C-115, 171, 255

Arendal C-239

Bosekop C-24

Lillestrom

Kjeller C-127, 189, 255

Nordi C.158

Oslo C-75, 216, 251

Tromső C.75, 125, 126, 189, 216, 255, 288, 293

Scandinavia C-189

Sweden C.236

Göteborg C-236

Kiruna C-89, 118, 136, 172, 173, 200, 255

Lycksele C-256

Uppsala C-256

Switzerland

Arosa C.115 
U. S. S. R. (General and European)

Moscow region

Moscow C.142, 195, 244, 294

Murmansk region C-144, 294

Tulom C-145

Leningrad region

Roshchino C.22, 192, 232

Uikraine

$$
\begin{aligned}
& \text { Sumy C.294 } \\
& \text { Yaroslavl region C.294 }
\end{aligned}
$$

5. North America C.113, 114, 265

Canada C.63, 66, 68, 104, 119, 154, 171, 196

Alberta

Meanook C.88

Manitoba

Winnipeg C-107

Northwest Territories

Resolute Bay C-107

Nova Scotia

Greenwood C-102

Ontario

Agincourt C.20

Ottawa C.20, 26, 62, 102, 132, 182, 187

Saskatchewan

Saskatoon C-67, 99, 101, 178, 179, 183-185, 197,255

Greenland C.221, 238, 257

Mt. Evans Observatory C.222

U. S. A. C. $6,96,260,265$

U. S. West Coast C-292 
Alaska C-6, 36, 169, 171, 223

Anchorage C-5

College C-5, 16, 17, 28, 36, 37, 167, 174, 211,

$233,241,285$
Point Barrow $\quad$ C-84, 156, 174

California

San Francisco C-257

Stanford C-88, 174, 229

District of Columbia

Anacostia

Bellevue C-260

Hawaii C-33

Honolulu C-257

Iowa

Cedar Rapids C-28, 83

Massachusetts

Cambridge C.230

Concord C-70

Newton Center C-230

S. Dartmouth C-106

Minnesota

Crow River C.20

Minneapolis C-289

New Jersey

Tuckerton C-230

New York C-135

Ithaca C-28, 83

Long Island

Riverdale C-153

Sampson Station C.96

Schenectady C.230

North Dakota

Bismarck C-174 


\section{Oregon}

$$
\begin{aligned}
& \text { Corvallis C.174 } \\
& \text { Pennsylvania } \\
& \text { Pittsburgh C.230 } \\
& \text { Washington C-257 } \\
& \text { Pullman C.83 }
\end{aligned}
$$

6. West Indies

Puerto Rico

San Juan C.153

Antigua C.85

7. South America C-265

Argentine

$$
\text { Buenos Aires C-135 }
$$

Brazil

Rio de Janeiro C-135

C. OCEANS

1. Indian Ocean C-180

2. Pacific Ocean

N. Pacific C.33

N. W. Pacific C-33

S. W. Pacific C.244 


$$
\operatorname{xxix}
$$

\section{CHRONOLOGICAL INDEX}

$\underline{1383}$

C-137

1396

C.23

1901

C.24

$\underline{1523}$

C-138

1925

C.266

1927

C.221

$\underline{1928}$

C. $8,51,201,230,251,252$, $250,267,274$

$\underline{1929}$

C. $37,222,253,263,268$

$\underline{1930}$

C $-41,135$

1931

C-157, 224, 254, 269

1932

C.76

1934

C.27, 161
1935

C. $-117,257$

1936

C. 124,217

1937

C-292

1933

C. $9,12,13,18,71,72$, $153,155,177,239,276-$ $278,287,288$

$\underline{1939}$

C.15, 39, 115, 123

$\underline{1940}$

C $-74,94,125,249$

1941

C.95

1942

C- 126,250

$\underline{1944}$

C.264

1946

C. 75,105

$\underline{1947}$

C- $61,141,146,176,231$, $241,285,295$

1948

C-195 
$\underline{1949}$

$$
\text { C }-93,97,291
$$

$\underline{1950}$

$$
\text { C }-\frac{7,10}{265}, 65,98,99,258,
$$

1951

$$
\begin{gathered}
\text { C - } 52,70,110,172,173, \\
205,206
\end{gathered}
$$

$\underline{1952}$

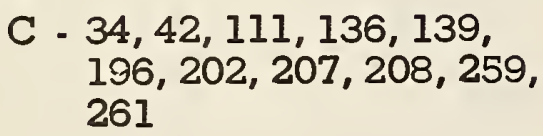

1953

$$
\begin{aligned}
C- & 33,47,50,53,67,100, \\
& 112,140,162,182,197, \\
& 234,262
\end{aligned}
$$

$\underline{1954}$

$$
\begin{aligned}
& \text { C }-2-4,35-37,46,54,59, \\
& 64,66,68,81,101,119, \\
& 127,134,183,184,190, \\
& 193,198,199,216,226, \\
& 229,293
\end{aligned}
$$

1955

$$
\begin{gathered}
C-28,38,43,44,48,82-84, \\
113,114,143,148,174, \\
185,218,255,296
\end{gathered}
$$

1956

$$
\begin{gathered}
C \cdot 5,29-31,55,69,132,149, \\
\quad 156,163,169,175,219, \\
223,245,246,275
\end{gathered}
$$

\section{7}

C - $11,21,45,56,57,90,102$, $106,144,150,159,180$, $211,214,215,236,238$, $247,270,283,294$
1958

$$
\begin{gathered}
\text { C }-6,19,25,26,40,49,62, \\
79,96,121,122,129, \\
133,142,145,151,154, \\
158,160,164,165,171, \\
178,186,189,192,194, \\
200,210,232,242-244, \\
248,256,271,284,289
\end{gathered}
$$

1959

C - $14,16,58,60,63,77,80$, $85,91,92,107-109,120$, $128,152,166,167,170$, $212,213,220,225,227$, $228,233,235,237,240$, $272,273,286$

$\underline{1960}$

C - $1,20,22,39,78,88,89$, $103,104,116,118,130$, $131,147,179,181,187$, $191,209,279,280,290$

1961

C - $17,86,188,203,204$, $281,282,297$ 
BIBLIOGRAPHY ON AURORAL RADIO WAVE PROPAGATION

C-1 Aarons, J. (Air Force, Cambridge Res. Cent.); Gustafsson, G. and Egeland, A. (both, Kiruna Geophys. Observ.), Correlation of audio-frequency electromagnetic radiation with auroral zone micropulsations. Nature, London, 185(4707): 148-151, Jan. 16, 1960. 5 figs., table, 6 refs. DWB--There are excellent correlations between the auroral zone micropulsations and the radiation in the audio-frequency band. Two distinct frequency bands have appeared on $12 \%$ of the days for which results were obtained. This is a lower limit since on several days when the sensitivity was low, emissions were not detected. The upper frequency band is "hiss". It varies in frequency throughout the recording period. The lower band at 750 C.P.S. for almost all the cases is steadier in frequency. Its spectral location indicates that it may well be radiation from protons, but its occasional change in frequency may make it another component of the "hiss" signals. (Item 12 A-1, Met. Abs.)--E. Z. S.

C-2 Agy, Vaughn, The location of the auroral zone. Journal of Geophysical Research, 5ऽ(2):267-272, June 1@54. 7 figs., 2 refs. --A study of data from a chain of radio-wave field strength recording stations along the SOth meridian suggests that "the auroral absorption zone" coincided ivith the zone of maximum frequency of occurrence of visible aurora. North of this, there appears to lie a region in which absorption is extremely low. Although the accuracy of location of the absorption zone is limited by use of fixed transmitters and receivers, as well as by the fact that varying modes of propagation are not taken into account, the indications are that it is somewhat farther north and narrower than has been suggested previously. (Item 6. 2-340, Met. Abs.).-Author's abstract.

C.3 Agy, Vaughn (Nat. Bur. of Standards, Boulder, Colo.), Geographic and temporal distribution of Polar blackouts. Journal of Geophysical Research, 59(4):4SS-512, Dec. 1554. 22 figs., 2 tables, 14 refs., eq. MH.BH--Tabulations of hourly values of the ionospheric parameters for 18 Northern Hemisphere stations have been used to derive diurnal variations in the 
occurrence of "blackout" conditions. Contour plots are presented showing the diumal average percentage of time during which blackout conditions prevailed, the amplitude of the diumal variation, and the time of maximum frequency of occurrence. Changes in the contours with season and with magnetic activity are discussed. (Item 6.8.22, Met. Abs.).. Author's abstract.

C.4 Agy, Vaughn, Variations in the probability of occurrence of polar blackouts. American Geophysical Union, Transactions, 35(2), 1954.--Tabulations of hourly values of the ionospheric parameters for 18 northern hemisphere stations have been used to derive diumal variations in the occurrence of "blackout" conditions. Geographic contour plots of the "probability" of occurrence are presented showing the average value, the amplitude of the diumal variation, and the time of maximum probability. Changes in the contours with season and with magnetic activity are discussed. Shortcomings in the method are emphasized and further studies are mentioned which may lead to conclusions of greater reliability and usefulness. (Abstract only).

C.5 Agy, Vaughn, Study of auroral zone attenuation of high frequency radio waves. U.S. National Bureau of Standards. Boulder Lab. , Colo. , Contract IA-454, Progress Report, April 1-June 30, 1956. Also Progress Report, July 1-Sept. 30, 1956 (both pub. June 14, 1957.) 2 pieces, each about 7 p. figs., 31 refs. in first report. DWB.-Correlation of field strength for reception of $8,10,11$ and $15 \mathrm{Mc}$ :vaves between Washington, D. C. , Bismarck, N. D., and Maui, respectively, and Anchorage and Fairbanles, respectively, is high enough to indicate that little difference exists (at laast did in Sept. 1955-June 1956), between transmission conditions over an auroral and a nonauroral path. Perhaps at times of sunspot maximurn or solar flares there would be some difference in conditions over the 2 types of paths. It is concluded that amount of auroral zone attenuation is much less than was previously supposed. (Item $\subseteq .10-304$, Met. Abs. )--M. R.

C.6 R.gy, Vaughn (National Bureau of Standards, Boulder, Colo.), Polar blackout occurrence patterns. Journal of Atmospheric and Terrestrial Physics, London, Special Suppl., Pt. 2, 1057, publ. 1958. p. 129-134, 4 figs. DLC..Presents maps of maximum probability of blackout on magnetically quiet and disturbed days, and shows the latitudinal variation in a graph. Suggests one type of blackout as due to magnetic activity, another to X-rays of auroral origin. (Item 11E-2, Met. Abs.) -.G.T. 
C-7 Alfven, Hannes, Magnetic storms and aurorae. (In his: Cosmical electrodynamics. Oxford, Clarendon Press, 1950. Chap. VI. p. 175-207. 20 figs., 26 refs., 27 eqs. DLC.Opens with the statement that "The intimate connection between magnetic storms and aurorae makes it necessary to consider them as two manifestations of the same phenomena". Reviews the work of BIRKELAND and STORMER which he considers the first serious attempt to interpret magnetic storms and auroras. He next summarizes the investigations of CHAPMAN and FERRARO. $\bar{A}$ more detailed discussion of the electric field theory (ALFVEN) and MALMFOR's scalemodel experiment follows. (Item 5C-140, Met. Abs.).. M. L. R.

Appleton, E. V., Note on "Short wave echoes and the aurora borealis by Van der Pol". Nature, London, 122(3084):37今. Dec. 8, 1928. fig. --Brief comments on Störmer's letter on auroral radio echoes (See ref. No. C-253) which possibly may be due to deflection caused by the spinning earth. Delayed signals of same nature were first observed by Taylor and Young. (See ref. No. C-261)-.-W. N.

C-9 Arkhangel'skii, B. F. and Pabo, N. V., Rasprostranenie radiovoln $\mathrm{V}$ vysokilkh shirotakh. (Radio wave propagation in high latitudes.) Leningrad. Arkticheskii NauchnoIssledovatel'skii Institut, Trudy, v. 124, 1938. 88 p. 55 figs., 6 tables, refs, at end of each chapter. DLC--A very thorough treatment of all available data on Arctic tropos. phere and ionospheric radio propagation conditions and their disturbance by solar, magnetic, auroral and statis (sferics) activity. Actual recorder records, as well as derived diagrams, tables, and curves are presented. Numerous references are given to the sources (mostly 1932-1037 in Russian periodicals). Theoretical considerations are not neglected but applications to study of atmosphere - especially the upper layers - and the aurora, ionosphere, terrestrial magnetism and atmospheric electricity are emphasized. The study is based on data obtained in 1932/33 and 1034/35 at Tikhaia (Calm) Bay in Franz Josef Land, and other Polar Year stations in 1932/33. (Item 5 I-73, Met. Abs.).-.M. R.

C-10 Aspinall, A. and Hawkins, G. S., Radio echo reflections from the aurora borealis, British Astronomical Association, Journal, 60(5):130-135, April 1950. figs., plate, tables, 12 refs. DLC.-This paper describes some studies of the aurora borealis using contemporary radio techniques such as are 
employed in the investigation of meteors. A number of aurora formations occurring during 1949 have been identified by the characteristic echoes which they produce on $72 \mathrm{Mc} / \mathrm{s}$, and continuous observations on this frequency have disclosed periods of auroral activity. Auroral rays were found to have an electron density of $6 \times 105$ electrons/cc and height measurements confirm that these rays occur from 100 to $300 \mathrm{kms}$ above the earth's surface. Apparent radial velocities have been observed which are interpreted as random movements in the aurora. (Item 6.2-338, Met. Abs.)--Authors' abstract.

C.11 Bailey, D. K. (Page Communications Eng. Inc., Wash., D.C.), Disturbances in the lower ionosphere observed at VHF following the solar flare of Feb. 23, 1956 with particular reference to auroral zone absorption. Journal of Geophysical Research, Wash., D. C., 62(3):431-463, Sept. 1957. 4 figs., 4 tables, 65 refs., 19 eqs. DLC.-Observations at the time of the great solar flare of $\mathrm{Feb} .23,1956$ of oblique incidence signal intensities and simultaneous observations of the background cosmic noise were made at VHF for a number of high latitude communication links employing the ionospheric scatter mode of propagation. During the flare and for some hours afterward, all paths lay in the dark hemisphere. Virtually synchronously with the arrival of solar cosmic rays, a sharp signal intensity enhancement was observed, which is tentatively explainable if it is supposed that the first arriving solar cosmic rays were predominantly of positive charge. The unusually stable nighttime absorption which developed in one to three hours after the flare and the much greater following daytime absorption are explained in some detail as consequences of the deposition in the $\mathrm{D}$ region of moderately heavy solar atomic ions, such as calcium, having ionization potentials low compared with the normal atmospheric constituents. The absorption effects, which were limited to fairly high geomag netic latitudes, gradually died away over a period of several days. The absence of significant magnetic disturbance and unusual auroral activity for nearly 48 hrs after the flare is shown to be in accord with the suggested explanation of the absorption effects. Assymmetry about local noon was observed in the absorption effects on signal intensity, for which an explanation is suggested. (Item 1IF-6, Met. Abs.)--Author's abstract.

C.12 Bailey, V. A., Generation of auroras by means of radio waves. Nature, London, 142(3596):613-614, Oct. 1, 1938. fig., 6 refs. -. Wants to take a $500 \mathrm{kw}$ station at gyro frequency, and beam of 800 half-wave antennas, and light up the $90 . \mathrm{km}$ atmosphere fifty times as bright as the night sky. This done, he 
wants to use $10^{6} \mathrm{kw}$ and light the country up like full moon light. Hopes to reduce auto accidents. --L. A. Manning.

C.13 Bailey, V. A., On some effects caused in the ionosphere by electric waves. Philosophical Magazine, 26(176):425-453, Oct. 1938. 3 figs. , table, refs. in foot notes, 68 eqs... Gives a series of 10 formulas through which propagation con. stant can be determined by successive substitution. Considers interaction caused by gyro-waves. Calculates gyro-power necessary to cause a glowing E layer. .-L. A. Manning.

C-14 Barbier, Daniel, L'observation des aurores polaires et de la lumière du ciel noctume pendant l'Ännee Geophysique Interna. tionale. (Observation of polar auroras and night airglow during the IGY.) La Nature, Paris, No. 3294:439-443, Oct. 1959. 5 figs. DLC.-The article presents a brief report on the morphology of polar auroras and night airglow, and considerations relating to the observation of these two phenomena during the IGY. The methods of auroral detection are described and also the methods used at the Observatory of Haute Provence (France) which led to discovery of a new phenomenon called "monochro. matic arc". The author describes this phenomenon and treats briefly of radioelectric exploration of auroras, artificial auroras, exploration of auroras by rockets and of the spectrum of auroras. Auroral exploration by rockets has shown that the auroras producing radiation particles are essentially electrons with energies less than $100 \mathrm{kev}$. Fast electrons, when decreasing speed, cause $\mathrm{X}$ radiation and play an important part in aurora production. Spectral auroral observation reveals some particular spectra, formed by atomic rays of neutral and ionized oxygen and neutral and ionized nitrogen. It shows that hydrogen rays may occur one or two hours before the beginning of the aurora and that there is a correlation between their extent and the presence of radioelectric echoes. (Item 11.5-125, Met. Abs.) ..A. V.

C-15 Barlow, E. W., Aurora and allied phenomena. Marine Observer, 16(133):12-17, Jan. 1939. table...A study of aurora and allied phenomena with brief mention of radio effects. During an aurora in 1915 radio reception in the British Isles was seriously affected, short wave reception being either completely absent or very weak and subject to fading. European stations near the lower end of the medium waveband were also faint. Telegraphic and telephonic communications were strongly interfered with. --E. Z. S. 
C-16 Bates, Howard F. (Geophys. Inst. Univ. Alaska, College, Alaska), The height of $F$ layer irregularities in the Arctic ionosphere. Journal of Geophysical Research, Wash., D.C. , 64(9):1257-1265, Sept. 1959. 5 figs., 10 refs., 14 eqs. DLC. Also issued as Alaska. Univ. Geophysical Institute, Contract AF 19(604)-1859, Scientific Report No. 3, March 1959. 19 p. 6 figs., 12 refs., 17 eqs. DWB--Results and interpretations of oblique-incidence soundings of the Arctic ionosphere are presented. Anomalous echoes are found to be prevalent in high latitudes in contrast to lower latitudes where 2F ground scatter predominates. One of the echoes seen regularly at College, Alaska, has been identified as direct $F$ layer (IF) back scatter propagated via the least time mode. The observations of the $1 F$ echo provide direct evidence of the presence of irregularities in the $F$ layer between heights of 350 and $500 \mathrm{~km}$. The $1 \mathrm{~F}$ echoes are recorded regularly at night and occasionally during the day in disturbed periods. They appear to be associated with auroral ionization. The analysis of ground scattered $(2 F)$ echoes is extended from a plane to a spherical geometry, and it is shown that a geometrical extension of the plane-earth theory is adequate. The observed range-frequency dependence differs only slightly from that predicted by the latter theory. (Item 11F-7, Met. Abs.)--Author's abstract.

C-17 Bates, Howard F., The slant Es echo. A high-frequency auroral echo. Journal of Geophysical Research, 66(2):447154, Feb. 1961. 6 figs., tables, 13 refs... Whenever the slant Es echo was strong on the College oblique incidence, sweep frequency (1-25 Mc/s) sounder, the College $41 \mathrm{Mc} / \mathrm{s}$ auroral radar recorded an echo at the same range as that at $25 \mathrm{Mc} / \mathrm{s}$ on the sweep frequency record. This range was close to that at which the line of sight most nearly approaches normal incidence upon the geomagnetic field lines. The highlatitude slant Es echo appears to be the result of energy that is scattered by randomly distributed, field aligned irregularities, and is strongly enhanced by one of two focusing agents that depend upon the frequency. The slanting portion of the echo is produced by least-time focusing, and the constant range VHF portion, by aspect focusing. The high latitude slant Es echo is associated with magnetic disturbances; in the cases examined the magnetic $K$ index was between 3 and 7. Its extension at $41 \mathrm{Mc} / \mathrm{s}$ is occasionally observed as a discrete type of auroral echo. The scatterers are diffusely distributed, however, indicating that auroral radar records must be interpreted with care.--Author's abstract. 
C.18 Beckman, B.; Menzel, W. and Vilbig, F., Veränderungen in der Ionosphäre beim Auftreten von Nordlicht. (Changes in the ionosphere during aurora borealis). Telegraphen Fernsprech Funk und Fernseh Technik, 27(7):245-25l, July 1938. 8 figs... Echo recordings of Sept. 30, 1937 using $84 \mathrm{~m} \lambda$ are discussed in comparison with simultaneous recordings conducted $100 \mathrm{~km}$ farther north. It is concluded that the auroral layers cannot be explained by reflexion from a northerly located slant ion-front. Over head changes of the $F$ region are distinct. Observed reflexions from $E, F$ and $F I$ and $F 2$ refute their theoretical nonexistence. .. W. N.

C.19 Bellchambers, W. H. and Piggott, W. R., Ionospheric measurements made at Halley Bay. Nature, London, 182(4649): 1596.1597, Dec. 6, 1958. fig. , 2 refs. .-An analysis of the initial results from the ionospheric sounding equipment at Halley Bay is given. Absorption controlled by corpuscular radiation during the polar winter has a maximum at $0400 \mathrm{~L} \mathrm{MT}$ $\left(30^{\circ} \mathrm{W}\right)$. The occurrence of auroral radar echoes shows a similar diumal maximum which peaks a few hours earlier; coincident with excessive ionospheric absorption and zenithal au. rora's weak reflections from an ionized layer at about $80 \mathrm{~km}$ have been obtained. The diumal variations of $f_{0} F 2$ show: (1) a peak near noon in the winter in spite of the comparative absence of photoionization; (2) a seasonal maximum near noon at the equinoxes; and (3) a minor increase around midnight during the summer. The results demonstrate the predominating influence of ionization transport phenomena over that of pre. viously mentioned processes. ..Phys. Abstract.

C.20 Bhattacharyya, B. K. (Natl. Res. Council, Ottawa), Correlation studies of radio-aurora, magnetic and earth-current disturbances. Canadian Journal of Physics, Ottawa, 38(5):624. 637, May 1960. 7 figs., tables, 20 refs. DWB, DLC.-Correlation studies of the radar echo occurrence rate from aurora in half-hourly intervals at Ottawa, $S$ and $S_{d}$ components of the horizontal magnetic field $\mathrm{H}$ at Agincourt, and the disturbance diumal variation of earth current at Crow River have been carried out. Short-time variations in auroral echo strength and moderate perturbations in $\mathrm{H}$ have also been correlated. The auroral echo occurrence rate seems to have a diurnal variation characteristic similar to that of $\mathrm{H}$. It is found that auroral activity always precedes magnetic activity. The variation of the delay time between the two phenomena shows a local time dependence, being practically constant and quite small (0.15 minutes) before local midnight and increasing afterwards. This variation of the delay time appears to have a connection with reports of others regarding reversal of the direction of auroral ionization drift from west to east somewhere around midnight 
with subsequent magnetic perturbations which change from positive to negative. No definite conclusion could be reached regarding the relationship of earth current to other factors because of a practically random variation of cross correlation coefficients from month to month. (Item 12A.28, Met. Abs.) -.Author's abstract.

C.21 Birfel'd, Ia. G., Radiolokatsionnye otrazheniia ot poliarnykh siianił. (Radar reflections from auroras.) Akademiia Nauk SSSR, Izvestiia, Ser. Geofiz., No. 4:543-547, 1957. 5 figs., 5 refs. DLC. Transl. into English by M. G. Priestley in the English language edition of the Izvestiia, Bulletin of the Academy of Sciences of the U.S.S.R., Geophysics Series, No. 4: 154-158, 1957, issued 1957, by American Geophysical Union. DWB--Radar reflections from auroras were studied with radar having a frequency of about $10 \mathrm{mh} \mathrm{Hz}$ for an impulse with length of $10 \mathrm{mc} / \mathrm{sec}$, a thickness of $75 \mathrm{kw}$ in a frequency of recurrence of $50 \mathrm{z}$. The type of reflections obtained on the radar screen are reproduced and analyzed. In general, the reflections, although complex, consist of signals having a width of the order of $10 \mathrm{mc} / \mathrm{sec}$ corresponding to the length of the impulse studied. The reflections are associated with solar activity and geomagnetic and ionospheric disturbances pro. duced in the ionosphere. Reflections were observed in all directions. (Item 10.8-129, Met. Abs.)--I. L.D.

C.22 Birfel'd, Ia. G. , Radiolokatsiia poliarnykh siianil. (Radar observation of auroras.) Akademiia Nauk SSSR, Izvestiia, Ser. Geofiz., No. 12:1871-1882, Dec. 1960. 7 figs., 32 refs. DLC. Transl. into English in the corresponding number of the Akademiia Nauk SSSR, Bulletin of the Academy of Sciences, USSR, Geophysics Series. DLC.-Various recording apparatuses (radar, oscillographs, photo cameras, etc.), re. ceiving equipments (multivibratory, semi-wave vibratory and parabolic antennas) and method of observation and coordination of the aurora are described with details and illustrations. The observations were made on six radar stations (Lopark, Dickson, Shmidt, Tixy, Roshchino and Yakutsk) on various frequencies from 5 to 74 megahertz. The character and properties of the radar single and group reflections and their typical classification are discussed with photographs, oscillograms and charts with graphic distribution of the ionization density and brightness of image on the semi-sky maps. General process of radar reflection and density of ionization are analyzed with respect to their relation to the solar activities. The radar group reflections of the auroras are graphically co. ordinated with $(\mathrm{H})$ and $(\mathrm{Z})$ components of the earth's magnetic field and also with variation of critical frequencies of $(E)$ and 
(F2) layer of the ionosphere. Major points of the discussion are summarized in the conclusion. --N.P.S.

C-23 Birkeland, Kristian, Sur les rayons cathodiques sous l'action de forces magnétiques intenses. (On cathode rays under the action of intense magnetic forces.) Ärchives des Sciences Physiques et Naturelles, Geneva, Ser. 4, 497-512, 1896. DLC--Laboratory experiments on cathode rays in tubes under the influence of magnetic fields are described in detail and the analogy to auroral manifestations suggested. The polarity of the aurora and the source of the energy are the two aspects that could be explained only on the basis of a hypothesis of polar origin of energy and action of magnetic poles in concentrating the discharges. (Item 5C-6, Met. Abs.).-.M. R.

C.24 Birkeland, Kristian, Expedition Norvegienne de 1899.1900 pour l'etude des aurores boreales. Resultats des recherches magnetiques, (Norwegian Expedition of 1899-1900 to study the aurora borealis. Results of magnetic research.) Videnskabs-selskabet i Kristiana, Math. -Naturvidenskapelig Klasse, Skrifter, No. 1, 1901. 80 p. 12 plates (some fold.), port. DLC..-Report contains discussion of simultaneous varia. tions in geomagnetism at Bosekop and Potsdam and relation to aurora and upper atmospheric electric currents. Theory is de. veloped and laboratory experiments illustrating the aurora in a cathode ray tube (artificial auroral bands) described. Finally, a discussion of possibility that cirrus clouds could be formed by extension of auroral action into lower levels of upper atmosphere is presented in some detail. (Item 5C-9, Met. Abs.) ..M. R.

C.25 Blevis, B. C. , UHF auroral radar observations. Joumal of Geophysical Research, 63(4):867-868, Dec. 1958. 2 figs. , 2 refs. .. Brief note on measurements with a bistatic type radar, transmitter receiver separation of about $19 \mathrm{~km}$, as conducted at Ottawa, Canada, during April 18.19, 1957 when auroral echoes were obtained on $488 \mathrm{Mc} / \mathrm{s}$ and an off-perpendicular angle corresponding to $3^{\circ}$ or $4^{\circ}$ between radar and magnetic lines of force at $100 \mathrm{~km}$ level. Echo observations at $944 \mathrm{Mc} / \mathrm{s}$ during July $8-9$ indicated effective cross sections up to several thousand $\mathrm{m}^{2}$...W. N.

C.26 Blevis, B. C. and Cameron, E. A. , Further data on radar returms from aurora at $488 \mathrm{Mc} / \mathrm{s}$. Canada. Defence Research Telecommunications Establishment, Report 44.2.2, July 1958. 9 p. 7 figs., ref. DWB (M(055) C212rep).-This report extends the information previously presented (Chapman, J.H. etal.) on auroral radar observations at a frequency of $488 \mathrm{Mc} / \mathrm{s}$. 
The present work is concerned primarily with measurements of auroral depth in range and with observed Doppler shifts in the signals returned from the aurora. The observations were made with a transmitter located near Ottawa, Ont. $\left(45^{\circ} 21\right.$ 'N, $75^{\circ}$ $53^{\prime} W$ ) and with the receiver located near Meath, Ont. ( $45^{\circ}$ $\left.44^{\prime} \mathrm{N}, 76^{\circ} 59^{\prime} \mathrm{W}\right)$. The equipment used for the measurements is described with particular reference to modifications which have been made since the previous report was published. Comments are made about the auroral echo geometry and the receiver and transmitter sites. Representative photographs of visual displays are included and the results of the measurements are indicated. (Item 12B-26, Met. Abs.)-.Authors' introduction.

C.27 Bontch-Bruewitch, M. A. , Ionospheric measurements in the polar regions. Nature, London, 133(3353):175-176, Feb. 3, 1934. -. Found evidence of an absorbing layer below the $E$ layer. -.L. A. Manning.

C-28 Booker, H. G.; Gartlein, C. W. and Nichols, B. (Cornell Univ.), Interpretations of radio reflections from the aurora. Journal of Geophysical Research, 60(1):1-22, March 1955. 17 figs., 31 refs., eq. DWB..The literature on radio echoes from the au. rora is reviewed and results obtained at Ithaca, N. Y., College, Alaska, and Saskatoon, and from analysis of radio signals transmitted from Cedar Rapids, Iowa, are analyzed. Diurnal and seasonal variations in auroral reflections correspond with frequency of auroras; maximum before and after midnight; minimum during daylight and at midnight; and, respectively, maximum at equinoxes (especially spring equinox), and minimum at solstices (especially winter solstice). Results of both pulse and continuous radio transmissions at 2.4 to $144 \mathrm{Mc} / \mathrm{sec}$ show fine structure, a zimuth (always to $\mathrm{N}$ ), fading time (proportional to frequency), which is much faster than would be expected and give clue to cause of above phenomena. Reflections are due to scattering from numerous ionized columns as in meteor trail reflections but with faster motion. Theory of ground reflection (HARANG) rejected. (See ref. C-127). (Item 6. 11-107, Met. Abs.) --M. R.

C-29 Booker, H. G., Some practical aspects of auroral propagation. Institute of Radio Engineers, Transactions on Communication Systems, CS-4(1):5, March 1956. S refs. DLC--The several aspects of the reflection or scatter mechanism which enable VHF radio communication up to $1000 \mathrm{~km}$ and $2000 \mathrm{~km}$, with low and high power transmitters respectively, are explained (except Doppler shift and fading) in terms of radio scattering under given conditions. It is advised to think of the regular VHF scattered transmission as the normal mean for communication 
in the auroral zone, and to use highly directional antennas and improved design of the modulation system. .-W. N.

C-30 Broker, H. G. , A theory of scattering by nonisotropic irregularities with application to radar reflections from the aurora. Journal of Ätmospheric and Terrestrial Physics, London, 8(4/5):204-221, May 1S56. 4 figs., 14 refs., 48 eqs. DWB. Also in: Cornell Univ., School of Electrical Engineering, Ithaca, N.Y., Technical Report 28, Oct. 30, 1955. --Radar echoes from aurora come mainly at low elevations from north, even at stations north of auroral visual zone. Horizontal and vertical extent of echoes increase as frequency decreases from 100-25 Mc/s. An explanation is given in terms of colurnns of ionization parallel to earth's magnetic field, but only about $40 \mathrm{~m}$ long by $1 \mathrm{~m}$ diameter, probably too small to be associated with visible rays. These irregularities are of the same order of size as turbulence elements and it is suggested that the magnetic field creates nonisotropic irregularities of electron density in the $E$ region, with maxima some 100 times the normal, but this cannot explain fading of auroral echoes, which must be due to other features of auroral ionization. (Item 7. 10.297, Met. Abs.).-C.E. P. B.

C-31 Booker, H. G. (Cornell Univ.), Turbulence in the ionosphere with applications to meteor trails, radio star scintillation, auroral radar echoes, and other phenomena. Joumal of Geophysical Research, Wash., D. C., 61(4):673-705, Dec. 1956. 11 figs., 43 refs. , 63 eqs. (Also in: Polar Atmosphere Symposium, Oslo, July 2-8, 1056, Proceedings, Pt. 2, Ionosphere Section, issued 1058. p. 52-81. 11 figs., etc.) These proceedings also issued as Joumal of Atmospheric and Terrestrial Physics, London, Special Supplement, Pt. 2 1957, pub. 1958. p. 52-81. DLC.-Two scales of turbulence are discussed, since large eddies are responsible for forward scattering phenomena and small eddies for back-scatter. Molecular diffusion theory forms the basis for small scale eddy formulas; Richardson's number is used for the large scale eddies. Large eddies have time constants of 40 to 100 seconds; small eddies about 0.4 səc near $100 \mathrm{~km}$. Large eddies have a scale of $1.6 \mathrm{~km}$, small eddies about $1.3 \mathrm{~m}$. The coefficient of eddy diffusion in met. eor trails is less than 10 for small eddies and increases to 104 for large eddies. The large eddies responsible for radio star scintillation are located near $200 \mathrm{~km}$. The turbulence power in watt $/ \mathrm{kg}$ is given as $5 \times 10^{-4}$ in the troposphere, 25 at the meteoric level and 1000 at the scintillation level. (Item 12B-31, Met. Abs.)--S. Fritz. 
C-32 Booker, Henry G., Radar studies of the aurora, (In: Ratcliffe, J. A. "Physics of the Upper Atmosphere". N. Y. Academic Press, 1960.) p. 355-375. 17 figs., table, 29 refs. -. A chapter devoted to discussion of radar studies of aurora under the following headings: Pulse radar experiments; Radar observations of the aurora; Analysis of observations in terms of azimuth; The distribution of auroral echoes with range; Analysis of auroral echoes in terms of height; Diurnal, sea. sonal, and sunspot cycle variations of auroral echoes; Motion associated with auroral echoes; Frequency dependence of auroral echoes; Polarization of auroral echoes; The importance of approximate perpendicularity between the Earth's magnetic field and the radius from the radar to the aurora; Theories of auroral radio echoes; and The cause of movement in the location of auroral echoes. --E. Z.S.

C.33 Bouchard, Jean, Sur la propagation ionosphérique des ondes décamétriques dans les régions polaires arctiques. (On ionospheric propagation of $10 \mathrm{~m}$ waves in the Arctic.) Académie des Sciences, Paris, Comptes Rendus, 236(2):220-222, Jan. 12, 1953. DLC--Systematic study of the propagation of $10 \mathrm{~m}$ waves over great distance shows the influence of ionic disturbances in the Arctic, connected with magnetic disturbances. The signals received at Dijon, France, from the region of the North Pacific (NE Siberia, Alaska, British Columbia, the Pacific Northwest and Hawaii) are distorted when passing through the belt of maximum auroral activity during magnetic storms but signals from other parts of the Pacific (Japan, Mariannes, New Zealand, etc.) are not thus distorted, except when taking sec. ondary paths traversing these disturbed zones. (Item 6D-158, Met. Ébs.)--M. R.

C-34 Bowles, $\mathbb{K}$. , The fading rate of ionospheric reflections from the aurora borealis at $50 \mathrm{mc} / \mathrm{sec}$. Journal of Geophysical Research, 57(2):191-196, June 1952. 3 figs., 2 refs. MH-BH... Amateur radio operators communicate from time to time by a phenomenon of anomalous ionospheric propagation associated with the aurora borealis. Measurements have been made of the rapid flutter-type of fading associated with this kind of propagation at $50 \mathrm{Mc} / \mathrm{sec}$. The power spectrum of the fading seems to have frequency components of roughly equal strength from zero frequency to a cut-off frequency between 100 and 200 cycles/sec. The fading frequency is thus about a power of ten greater than might be anticipated from usual ionospherio velocities. In most cases, both transmitting and receiving antennas must be of the same polarization and must be pointed more or less in the direction of the visible aurora. Amplitude modulated speech transmission is occasionally possible at 50 $\mathrm{Mc} / \mathrm{sec}$ by means of this type of propagation. (Item 4.5.233, Met. Abs.)-.Author's abstract. 
C.35 Bowles, K. L., Analysis of the fading of very high frequency radio waves propagated by aurorally disturbed ionosphere. Cornell University, School of Electrical Engineering, Studies on propagation in ionosphere, Technical Report, No. 15, 1954. Master Thesis. (Unchecked).

C-36 Bowles, K. L. (Geoph. Inst., Univ. of Alaska), Doppler shifted radio echoes from aurora. Journal of Geophysical Re. search, 59(4):553-555, Dec. 1954. fig., 5 refs. MH-BH.. A brief report on observations and discoveries regarding the Doppler shift in radio echoes from the aurora using $25.4 \mathrm{Mc} /$ sec C. W. or pulse signals from a 100 watt transmitter located $15 \mathrm{mi}$ from a low-gain receiving antenna pointed $\mathrm{N}$. The spectrum of the Doppler shifted auroral echo and fo signal from transmitter obtained at College, Alaska, Oct. 24, 1954 is reproduced. Doppler shifts cannot be attributed to horizontal movements. Upward shifts correlate with homogeneous auroral forms; (downward motion) and downward shifts with raus (upward motion). Velocities along lines of force are calculated to be about $6 \mathrm{~km} / \mathrm{sec}$ which is much less than MEINEI reports obtaining from optical methods. (Item 6.8-348, Met. Abs.)...M. R.

C-37 Bowles, K. L., VHF auroral and meteor echoes including simultaneous observations. American Geophysical Union, Transactions, 35(2):375, 1954....Auroral echoes have been observed at College, Alaska, using SCR270 radar at $106 \mathrm{mc} /$ sec. The location of College close to the center of the auroral zone makes these observations unique, since visible aurora is commonly seen in all directions of azimuth, as well as overhead. Similar to previous results, (1) echoes are found only within a sector slightly wider than one quadrant, centered on geomagnetic north, (2) ranges between approximately $500-1000 \mathrm{~km}$ are found, and (3) no vertical incidence echoes may be obtained from the various forms of aurora when seen overhead. These observations are seen to conform with the meteor trail analogy for VHF echoes as postulated by R. K. Moore. An interesting observation, though not substantiated by a large amount of data, is that the rate and strength of meteor echoes is considerably enhanced during periods when aurora is seen in the sky. The normal rate of three to five echoes per hour is increased to as much as 25-30 echoes per hour. No indication of preferred azimuth is found. In the meteor echo after the initial whistle has ceased, the fading rate fits in with results of other workers but is an order of magnitude slower than fading found in auroral echoes. (See ref. C-208). 
C.38 Bowles, K. L. , Aurora borealis studies using VHF radio echoes. IRE-URSI Meeting, Washington, D. C., May 1955. -.Two experiments recently performed by the author are described. In the first a $100 \mathrm{mc} / \mathrm{s}$ radar set with good horizontal antenna beamwidth was used in Alaska. Simultaneous photographs of the visible aurora and the PPI pattern were taken. In many cases direct comparison may be made between the two. Echoes were received from both homogeneous and rayed auroral forms. A suggested accounting is made for similar visible forms which do not correlate with echoes. In the second experiment rf spectrograms were made of $\mathrm{cw}$ echoes from aurora at several carrier frequencies (between 25 and $150 \mathrm{mc} / \mathrm{s}$ ) and locations. A-'scope presentation of pulsed echoes was available at the same time for comparison with the spectra. Final analysis has been made from tape recordings of the two types of information. Whenever possible visual aurora was correlated with the radio echoes. The results show that the auroral echoes are generally Doppler shifted several hundred cycles relative to the transmitted frequency. They are also broadened over a band of several hundred cycles width. Both quantities depend upon carrier frequency and location of the station in the geomagnetic field. Homogeneous auroral forms produce upward Doppler shifts (approaching motion) while rayed forms produce downward shifts (receding motion). The results are taken as support for the suggestion of earlier Cornell work that the radio echoes arise from thin columns of ionization aligned with the lines of force of the earth's magnetic field. The motions implied by the Doppler shifts are downward in homogeneous forms but upward in rayed forms in the visible aurora. With accounting taken for geometry, velocities along the columns of the order of $6 \mathrm{~km} / \mathrm{s}$ are implied.

C.39 Bowles, K. L.; Cohen, R. ; Ochs, G. R. et al. (all, Natl. Bur. of Standards, Boulder, Colo.), Radio echoes from field aligned ionization above the magnetic equator and their resemblance to auroral echoes. Journal of Geophysical Research, Wash., D. C. , 65(6):1853.1855, June 1960. 6 refs. DLC.Both classes of echoes occur in about the same height range and have about the same band width of fading and similar Doppler shift characteristics. Both exhibit an aspect of sensitivity corresponding to the influence of the earth's magnetic field. Strong currents are observed to flow horizontally to the lines of force of the earth's magnetic field both at the magnetic equator and in auroral forms. The authors suggest that in both pilenomena irregularities could reasonably consist of a family of plane-wave disturbances constrained to lie parallel to the local lines of force of the earth's magnetic field. The apparent weakness of the aspect sensitivity in both cases could be pro. duced by local distortion of the earth's magnetic field due to the flow of strong localized ionospheric currents. (Item 12B32, Met. Abs.)--E.Z.S. 
C.40 Brown, Robert Hanbury and Lovell, A.C.B., Radio and the Aurora borealis. (In their: Exploration of space by radio. N. Y., John Wiley, 1958. pp. 171-177. 7 figs. (incl. photos), 2 refs.) DLC (QB475. B7)-.-A brief but fairly detailed discussion of radio investigations of auroras. Radio echoes and radio noise from auroras, electron density in the auroras, interpretation of the echo structure, motion of the echo pattern, and some associated magnetic, visual and scintillation fea. tures are the topics covered. Figures and photos illustrate the chapter, and references to the work of specific investigators are given in the text. (Item 12A-34, Met. Abs.).A. J. M.

C.41 Brilche, E., Modelversuche mit sichtbaren Elektronstrahlen zu Störmers Theorie des Polarlichtes und des Welraumechos. (Model experiments with visible electron rays to Störmer's theory on polar aurora and worldwide echoes.) Naturwissenschaften, 18(50):1085-1092, Dec. 12, 1930. 9 figs., 7 refs., eqs. .-An illustrated presentation of laboratory experiments with a magnetic sphere simulating the earth exposed to electron radiation (200-250 volts) from an oxide cathode, in order to check the trajectories of the electrons and their behavior in a dipole field. The experiments showed good agreement with Störmer's theory. (See ref. C-255)...W. N.

C-42 Budden, K. G. and Yates, G. G., A search for radio echoes of long delay. Journal of Atmospheric and Terrestrial Physics, 2(5):272-281, 1952. 23 refs. DWB.-An unsuccessful search was made for echoes delayed $3-15 \mathrm{sec}$. The cause of such echoes is discussed and they are tentatively attributed to "the propagation of guided waves over long curved paths formed by belts of ions outside the earth but fixed relative to it". (Item 4.4-100, Met. Abs.)--C.E.P.B.

C.43 Bullough, $K$. and Kaiser, T. R., Radio reflections from aurorae, $\mathrm{Pt}$. 1. Journal of Atmospheric and Terrestrial Physics, 5(4):189-200, Sept. 1954. 9 figs. , 3 tables, 16 refs. Also: Pt. 2, Ibid., 6(4):198-214, April 1955. 18 figs., table, 11 refs. DWB-.Pt. 1 reports that observations at Jodrell Bank with a rotating aerial system on $4.1 \mathrm{~m}$ included 3 occasions of auroral echo. Echo is interpreted as a reflection from the most highly ionized part of an auroral arc extending more than 1000 $\mathrm{km}$ along geomagnetic latitude $59^{\circ}$. The reflecting region was only a few $\mathrm{km}$ across, at a height below $125 \mathrm{~km}$, moving westward at $1.2 \mathrm{~km} / \mathrm{sec}$. The movement is attributed to movement of the ionizing agent. Pt. 2: Observations of auroral echoes 1949.53 on a beam directed $68^{\circ} \mathrm{W}$ of $\mathrm{N}$ at Jodrell Bank are attributed to large single blobs or localized aggregates. There was a marked fall of activity in 1952-53 with decrease of 
sunspot activity. Echoes without discrete structure occur mainly between $16 \mathrm{~h}$ and $21 \mathrm{~h}$, those with discrete structure between $18 \mathrm{~h}$ and $6 \mathrm{~h}$. Annual variation shows a slight maximum in July-Oct. Geomagnetic locality, correlation with visual aurora and with magnetic activity are discussed; auroral speeds and motions are described. (See ref. C-129). (Item 12B-35, Met. Abs.).-C.E.P.B.

C.44 Bullough, K. and Kaiser, T. R., Radio reflections from aurorae, $\mathrm{Pt}_{2}$ 2. Joumal of Atmospheric and Terrestrial Physics, 6(4):198.214, April 1955. 18 figs., table, 11 refs. DWB.. Observations of auroral echoes $1949-53$ on a beam directed $68^{\circ} \mathrm{W}$ of $\mathrm{N}$ at Jodrell Bank are attributed to large single blobs or localized aggregates. There was a marked fall of activity in 1952-3 with decrease of sunspot activity. Echoes without discrete structure occur mainly between 16 and $21 \mathrm{~h}$, those with discrete structure between $18 \mathrm{~h}$ and $6 \mathrm{~h}$. Annual variation shows a slight maximum in July-Oct. Geomagnetic locality, correlation with visual aurora and with magnetic activity are discussed. Evening echoes $(13.21 \mathrm{~h})$ moved westward, speed at $15 \mathrm{~h}$ being $600 \mathrm{~m} / \mathrm{s}$ to west, night-morning echoes $(22.6 \mathrm{~h})$ moved eastward, speed being $600 \mathrm{~m} / \mathrm{s}$ to east at $5 \mathrm{~h}$. At $21 \mathrm{~h}$, when echoes are at a minimum, mean speed is zero. (Item 6. 8-349, Met. Abs.)--C.E.P. B.

C-45 Bullough, K. ; Davidson, T. W.; Kaiser, T. R. and Watkins, C. D. , Radio reflections from aurorae, $\mathrm{Pt}$. 3 , The associa. tion with geomagnetic phenomena. Journal of Átmospheric and Terrestrial Physics, London, 11(3/4):237-254, 1957. 15 figs. , 2 tables, 15 refs. DLC--This paper presents a further study of the radio-echo observations of auroral ionization,made on a frequency of $72 \mathrm{Mc} / \mathrm{s}$ between June 1949 and Oct. 1953, described in a previous paper of this series (BULLOUGH and KAISER, 1955). The data are compared with geomagnetic observations made at Eskdalemuir during the same period. A good correlation is found between the daily frequency distribu. tion of the radio echoes and the mean daily variation in magnetic disturbance. The change from a positive to negative bay type of disturbance tends to occur between 2100 and $2200 \mathrm{hr}$ local time and coincides with a minimum in the frequency of echo occurrence. This minimum separates the diffuse echoes (from westward-moving ionization) of the evening sequence and the discrete echoes (from eastward moving ionization) of the morning sequence. There exists a close correspondence between the occurrence of radio echoes and features in the magnetic disturbance. In a number of cases there appears to be a displacement in time between echo components and peaks in the magnetograms; it is suggested that this corresponds to the time of transit of the ionization between the reflecting region 
and the magnetic meridian through Eskdalemuir (some $10^{\circ}$ in magnetic longitude to the west). The probability of occurrence of $72 \mathrm{Mc} / \mathrm{s}$ echoes during a positive disturbance is approximately double that for a negative one, being $50 \%$ for $\Delta H>100 y$ and $\Delta H<-200 y$. The results and conclusions appear in several aspects to conflict with the ChapmanFerraro-Martyn theory of magnetic storms and aurorae. (Item 12B-36, Met. Abs.)--Authors' abstract.

C-46 Burkhart, K., Radioechos am Nordlicht. (Auroral radio echoes.) Umschau, 54(11):342, June 1, 1954. 2 refs. DLC.Brief article on discrete and diffuse echoes. Radar echoes give the height of the auroral layer as $110 \mathrm{~km}$ with a range of not more than $5 \mathrm{~km}$. (Item 5.8-280, Met. Äbs.)-.C.E.P.B.

C-47 Cain, Joseph C. (Geophysical Institute of the Univ. of Alaska), Auroral radio-echo table and diagram for a station in geomagnetic latitude 56. Joumal of Geophysical Research, Wash., D. C., 58(3):377-380, Sept. 1953. fig., table, ref. DWB-CHAPMAN has computed the echo geometry of radio signals reflected from auroras for the transmitter-receiver sites at geomagnetic colatitudes, $\alpha=30^{\circ}, 45^{\circ}, 60^{\circ}$, and $50^{\circ}$. This paper computes the echo geometry for $\alpha=34^{\circ}$, which is the geomagnetic colatitude of the Jodrell Bank Radio Experimental Station, of the University of Manchester, and is very close to that of the stations in northern United States which are concerned with radio echo work. (Item 5C-235, Met. Abs.)..Author's abstract.

C.48 Chamberlain, Joseph W., Auroral rays as electric-discharge phenomena. Astrophysical Journal, Chicago, 122(2):349-350, Sept. 1955. 3 refs. DLC--Nearly constant luminosity of auroral rays over a large height and density range requires a compensatory mechanism which is not fully understood. Excitation could increase with height due to greater incident energy, and the number of collisions decrease with height due to decreasing density. A current density of $6 \times 1012$ electrons/ $\mathrm{cm}^{2} \mathrm{sec}$ would be necessary for a ray $10^{4} \mathrm{~cm}$ in diameter. An electron density of $10^{6} \mathrm{~cm}^{-3}$ would be postulated for the lower part of the ray (in arcs $10^{8}$ electrons $/ \mathrm{cm}^{3}$ ). The current density is uncertain because the diameter of rays is uncertain. The theory advanced by author balances the electron and proton influx and penetration against the conductivity in the layers where the arcs form and leads to the conclusion that the electron may be accelerated downward by the pull of the protons and that when arcs recede the rays are left in position originally occupied by the latter. (Item 7.10.258, Met. Abs.) --M. R. 
C-49 Chamberlain, Joseph W., Theories of the aurora. Advances in Geophysics, N. Y., 4:109-215, 1958. 21 figs., 113 refs., numerous eqs. DLC. Also reprinted as Chicago. Univ. Yerkes Obs., Williams Bay, Wisc., Contract AF 19(122)-480, Technical Report, No. 40...A major critical summary and re. view of current knowledge of auroral physics and theory. Historical and observational aspects comprise $\mathrm{Ch}$. 1 ; Motions of charged particles in magnetic fields, Ch. 2; STÓRMER's theory, Ch. 3; Electric currents from sun to earth, 4; CHAP. MAN-FERRARO's theory and ring current, 5; ALFVEN's, HOYLE's and LEBEDINSKII's theories, 6; VESTINE and WULF's dynamo theory, SINGER's shock-wave theory, PARKER's theory of hydromagnetic displacement of lines of force, MARIS HULBURT's UV-light and meteor theories, 7; and theories of auroral excitation, 8. (Item S.9.17, Met. Abs.)-.M.R.

C.50 Chapman, R. P. and Currie, B. W. (Physics Dept., Saskatchewan Univ., Canada), Radio noise from aurora. Journal of Geophysical Research, Wash., D. C., 58(3):363.367, Sept. 1953. fig., 13 refs. DWB.. $\bar{A}$ search for radio noise of $10 \mathrm{~cm}$ wavelength from aurora during 1951 and 1952 with improved equipment was unsuccessful. The failure to detect the auroral radio noise, observed previously in 1949, is attributed to the decrease of the intensity of auroral displays and of sunspot activity. (Item 5C-236, Met. Abs.)--Authors' abstract.

C-51 Chapman, Sydney and Eckersley, T. L., Radio echoes and magnetic storms. Nature, London, 122(308):768, Nov. 17, 1928. --Brief note on St"ormer's observation of auroral radio echoes at $\lambda 31.4 \mathrm{~m}$. (See ref. C-253). If streams of elec. trons emitted from the sun associated with aurora be the cause, as Störmer suggests, then this is the first direct evidence obtained of the density of the streamers since an electron density of $10^{5}$ to $10^{6}$ per $\mathrm{cm}^{3}$ is required for these reflected signals. -.W. N.

C-52 Chapman, Sydney, The theory of magnetic storms and aurora. Nature, London, 168(4263):86, July 14, 1951. 5 refs. ... $\bar{A}$ brief note suggesting the need for experimental work to test the various theories of magnetic storms and auroras...W. N.

C-53 Chapman, Sydney, The geometry of radio echoes from aurorae. Joumal of Atmospheric and Terrestrial Physics, 3(1):1.29, Jan. 1953. 8 figs., 3 tables, 9 refs., eqs. DWB-.An examination of discrete auroral echoes in which part of a radio beam is reflected back to sending station in Northern Hemisphere from aurora borealis, or possibly from an aurora australis stream beyond the atmosphere. The reflecting unit is taken to be an auroral ray lying along a line of force of the geomagnetic field. 
Diagrams and tables show positions of surfaces reflecting radio beams of different elevations at stations in different latitudes. (Item 4.9-34, Met. Abs.)--C.E.P.B.

C-54 Chapman, Sydney, Aurora and airglow. Report from Subject group IV, of International Union of Geodesy and Geophysics, at the 10th Assembly, Rome 1954. 7 p. refs. Mimeo... Objectives of aurora and airglow program suggested for IGY, 1957-8, are set forth and visual, photographic, spectroscopic, radio-auroral, rocket auroral and airglow measurements planned, are described in some detail. (Item 9.7-38, Met. Äbs.) -.M. R.

C-55 Chapman, Sydney, The IGY auroral programme. International Geophysical Year, 1957/1958, Comite Special, Bulletin d'Information, No. 7:58-61, 1956. DWB.-The objectives of auroral observations during the IGY are: an understanding of the nature of auroras, the solar emissions, the modes and periods of emis. sions from the sun, their courses to the earth and the ensuing luminous, electromagnetic and ionospheric processes near our earth and in the atmosphere. The immediate objective to increase our factual knowledge of the aurora. The auroral pro. gram during this IGY included the following: the synoptic picture by photographic methods and visual surveys, alerts and special world intervals for warning information, the photometry, spectrophotometry and spectrography, the possible transfer of equipment during the months of Arctic daylight, the radio detection of aurora, the auroral radio emissions, radio absorption and scattering associated with auroras, rocket studies, atmos. pheric electric field, auroral sounds. The principal experiments in the ionospheric program are included. (Item 10.6-34, Met. Abs. ) --N. N.

C-56 Chapman, Sydney and Little, C. Gordon, The nondeviative absorotion of high frequency radio waves in auroral latitudes. Journal of Atmospheric and Terrestrial Physics, 10(1):20-31, Jan. 1957. 3 tables, 21 refs., 14 eqs. DWB--In auroral lati. tudes the nondeviative absorption of high frequency radio waves is much more irregular, and often much stronger, than in subauroral latitudes. It is greater and more frequent by day than by night; this is the converse of the daily variation of magnetic activity. The electzons that produce the absorption in subauroral latitudes are mainly caused by solar ultraviolet light; in auroral latitudes, often the major source is bombardment of the atmosphere by solar gas. According to J. A. VAN ALLEN's new interpretation of the soft radiation observed by himself and his colleagues in auroral latitudes, down to $50 \mathrm{~km}$, a small minority of the primary bombarding particles generate X-rays, which penetrate further than the particles themselves. It is here 
suggested that the layer ionized by these $\mathrm{X}$-rays is an important factor in the daytime radio absorption. Also, as D. $R$. BATES has pointed out, Lyman-alpha radiation will be generated by the auroral protons; ionization of nitric oxide mole. cules by this radiation may also contribute appreciably to the absorption. The same processes of ionization will occur likewise at night, and often still more strongly; however, loss of the electrons to form negative ions by attachment is countered by photodetachment during the day, but not at night. Very tentative tables are given, based on these ideas, indicating ionospheric conditions consistent with greater daytime absorption than at night, even when the nighttime bombardment is twenty times more intense than that by day. The corresponding absorption relaxation times, and daily variation of mag. netic disturbance, will be examined in a later note. (Item 8.6-179, Met. Abs.)..Authors' abstract.

C-57 Chapman, Sydney, Auroral observation in India and Pakistan. National Institute of Sciences of India, Calcutta, Bulletin, No. 9:180-192, Nov. 2, 1957. fig., 10 refs. Abstracted from reprint. DWB (523. 74 C719rg)--Printed records remain of observations from at least eight places, in what is now India and Pakistan, of the great aurora of Feb. 4, 1872; their geographic latitudes range from $34^{\circ}$ to $19^{\circ} \mathrm{N}$ (geomagnetic, $24^{\circ}$ to $\left.10^{\circ} \mathrm{N}\right)$. Hence this remarkable aurora may be called tropical as well as polar. These records are reproduced and dis. cussed, and a plea is renewed for a search for further records of observations of this aurora (and possibly also others) from the Indian subcontinent. More important still, attention is drawn to the need for observation of future great auroras observable therefrom, and from elsewhere in low latitudes, and especially from high altitude observatories. The types of desirable observations and equipment are outlined. The possibilities of successful observation (on the rare occasions when nature provides the event) may be increased by keeping a special watch at times when there is an enhanced probability of their occurrence. Such times may be recognized with the aid of magnetic, radio and solar observations made on the spot, or they may be called attention to by an international center, as is planned during the International Geophysical Year 1957-58. (Item 10.11-265, Met. Abs.).-Author's ab. stract.

C-58 Chapman, Sydney (Geophysical Inst., College, Alaska), Disturbances in the lower auroral ionosphere. Journal of Atmospheric and Terrestrial Physics, London, 15(1/2):2C-37, Sept. 1359. 2 figs., table, 16 refs. DLC.-The solar particles that enter the atmosphere and produce the luminous aurora are known to include protons, which also ionize the atmosphere. 
As suggested by BATES, their ionizing action may extend below the level of their own penetration, by the Lyman $\alpha$ - photons they emit. These can penetrate to about $75 \mathrm{~km}$, and ionize nitric oxide. Recent rocket researches by VAN ALLEN and his colleagues prove that the primary auroral particles also in. clude electrons, with energies up to $100 \mathrm{keV}$. These pene. trate to about $80 \mathrm{~km}$, thus directly extending the auroral ionization well below the level of auroral luminosity. Indirectly these electrons ionize the atmosphere down to far lower levels, by the X-rays emitted by a small fraction of the electrons. WINCKLER's balloon results show that such ionization extends at least down to $32 \mathrm{~km}$. This ionization below the level of the visible aurora accounts for most of the absorption, in auroral regions, of high frequency radio waves. Though the primary electron flux at night probably exceeds that by day, the secondary electrons are often more numerous (and absorbent) by day than by night. This is because, by day, photodetachment prolongs the free life of these electrons, despite their ready attachment to oxygen. (Item 11F-18, Met. Abs.).. Author's abstract.

C-5e Chestnov, F. I., Zagadka ionosfery. (Enigma of the ionosphere.) Moscow, Gosud. Izdat. Tekhniko-Teoreticheskio Literary, 1954. 54 p. 24 figs. Nauchno-populiarnaia Biblioteka, No. 70. DLC.-The detailed structure of the ionosphere is described and shown in an original schematic diagram. Ionization, twilight, night sky light, aurora, diumal effects, magnetic field, magnetic storms and ionosphere, solar effects, radio propagation (long and short wave), ionospheric soundings by radio impulses, sunspot effects, forecasts of radio propaga. tion, meteor traces, radio radiation from stars and rocket exploration of ionosphere are treated in condensed popular tech. nical form. (Item 6D-235, Met. Abs.)--M.R.

C.60 Chivers, H.J.A. (Univ. of Manchester, Jodrell Bank Exper. Sta.) and Wells, H. W. (Carnegie Inst., Wash.), Observations of unusual radiofrequency noise emission and absorption at $80 \mathrm{Mc} / \mathrm{s}_{\text {a }}$ Journal of Atmospheric and Terrestrial Physics, N. Y., 17(1/2):13-19, Dec. 1959. 2 figs, , 2 tables, 11 refs. DWB, DLC.-Unusual radiofrequency noise emissions at $80 \mathrm{Mc} / \mathrm{s}$ have been identified during periods of solar activity. The noise enhancements may be classified as (1) smooth, bay-like disturbances lasting for approximately $l \mathrm{hr}$ which occur in both diıy and night hours, and (2) abrupt increases, often of large but fluctuating amplitude which occur within a few hours of local midnight. The smooth enhancements occur almost simultaneously with the absorption of radiation in a sector of the northern sky. These effects could be caused by transit of high velocity streams of charged particles which produce emission 
from $F$ region levels and absorption in the $E$ region or below. The abrupt noise bursts at night are from the northern sky and seem to be associated with pronounced changes in the horizontal component of the earth's magnetic field. The noise may be a form of "auroral" radiation or may arise from propagation of solar noise outbursts from the sunlit to the dark hemisphere. (Item 11. 7-164, Met. Abs.).-Authors' abstract.

C.61 Clegg, J. A. and Ellyett, C. D. , Radio echoes from the aurora borealis. Nature, London, 160(4063):372, Sept. 13, 1947. 6 refs. , eqs. -.Radio echoes from a distance of 480 miles were obtained on $46 \mathrm{Mc} / \mathrm{s}$, apparently arising from a luminescent cloud near the end of an aurora streamer. There was also general nonlocalized reflection from the same region on 46 and 72 $\mathrm{Mc} / \mathrm{s}$. The ionization density is estimated to have been $\sim$ $100 \mathrm{x}$ the average $\mathrm{F}$ layer night ionization. --Authors ' abstract.

C-62 Collins, C., Some observations of aurora using a low-power frequency modulated radar. Canadian Journal of Physics, Ottawa, 36(7):926-934, July 1958. 5 figs., table, 18 refs. DWB, DLC-. $A$ frequency modulated, continuous-wave radar seems to be particularly well suited to the observation of auroral ionization, since it provides both range information and a Doppler indication of radial motion. An experimental equipment of this type has been operated for a few months near Ottawa. The system parameters are briefly considered and the radar observations are compared with similar measurements made with higher powered pulse systems. (Item 10.5-164, Met. Abs.)--Author's abstract.

C-63 Collins, C. and Forsyth, P. A. (both, Defence Research Board, Ottawa, Canada), A bistatic radio investigation of auroral ionization. Journal of Atmospheric and Terrestrial Physics, London, 13(3/4):315-345, Feb. 1959. 13 figs., 2 ta. bles, 32 refs. DLC.-The scattering of radio waves in the upper atmosphere during auroral disturbances has been studied by means of a number of radio systems in which each trans. mitter was separated from its associated receiver by a dis. tance of about $1000 \mathrm{~km}$. Some twenty of these systems were used in a network covering a large area in Canada. The frequencies were in the range $30.50 \mathrm{Mc} / \mathrm{s}$. At least four different kinds of auroral events are distinguishable. Of these, two appear to be associated separately with different phases of visible aurora, the third with a later stage in the auroral process which is not observed visually, and a fourth with the recurrent daytime absorption which often precedes an auroral disturbance. The term "radio-aurora", which applies to all of the events, is used to avoid confusion with that part of the auroral process which is observable visually. In these four 
events, evidence is found for three separate scattering mechanisms, each of which has been proposed previously as the principal source of radar echoes from aurora. (Item 11.1-315, Met. Abs. )-.Authors' abstract.

C-64 Conference on Auroral Physics, July 23-26, 1951, London, Ontario, Proceedings. Ed. by N. C. Gerson; T. J. Keneshea and R. J. Donaldson, Jr. U. S. Air Force. Cambridge Research Center, Geophysical Research Papers, No. 30, July 1954.459 p. numerous tables, diagrs. (incl. photos) and eqs. Bibliogs. throughout. Alphabetical author and subject indexes. MH-BH-.-The papers collected in this volume have been abstracted separately. They cover the theory and physicochemical aspects of the aurora, sunlit aurora, radar or radio reflections from auroras, the airglow, laboratory investigations of upper atmospheric processes and auroral afterglow, interpretation of atmospheric emission, geomagnetic storms and aurora, solar and magneto-hydrodynamic waves and atmospheric absorption. Many references and a good index are included. (Item 6.9-3, Met. Abs.)--M. R.

C.55 Covington, A. E., Microwave sky noise. Joumal of Geophysical Research, 55(1):33-37, March 1950. 4 figs., 7 refs. MH-BH--Radiation of $2800 \mathrm{mc}$ was received from empty sky, equivalent to a temperature of ca. $50^{\circ} \mathrm{K}$ but with short time bursts from zenith. Examples are described, associated with magnetic disturbances, with aurora overhead and once with a sudden ionospheric disturbance. (Item 4.9.113, Met. Abs.) -.C. E. P. B.

C.66 Cox, J. W. and Davies, K., Statistical studies of polar radio blackouts. Canadian Joumal of Physics, 32(12):743-756, 1954. 15 figs., 3 refs. -. $\bar{A}$ statistical study of high-frequency radio blackouts in Canada is made from records taken at several ionosphere sounding stations. Both vertical incidence and communication data are examined to determine the geographical, seasonal and diumal distributions of the frequency of occurrence of blackout. It is found that blackouts are most abundant in the morning and that the time of maximum occurrence increases with increasing latitude. --A uthors' abstract.

C.67 Currie, B. W. ; Forsyth, P. A. and Vawter, F. E. (Physics Dept., Saskatchewan Univ., Canada), Radio reflections from aurora. Joumal of Geophysical Research, 59(2):179-200, June 1353. 7 figs., 5 tables, 19 refs. MH-BH--This paper outlines observations and experiences associated with a continuing investigation of radio echoes from aurora at Saskatoon, Canada. For the three frequencies investigated - 3000, 106 and $56 \mathrm{Mc} / \mathrm{s}$ - echoes have been observed with the last two. Echoes occur 
when the auroral forms exhibit some ray structure and, then, only from parts of the aurora at elevations less than $15^{\circ}$ above the horizon. Using range-amplitude and range-azimuth displays of echoes in combination with the corresponding photographs of the aurora, it is shown that the echoes originate from levels close to the lower edge of the aurora. Height dis. tributions of the centers from which the echoes come agree closely with height distributions found from parallactic photo. graphs of aurora in other regions. Using aerial arrays for the 106. and $56 \cdot \mathrm{Mc} / \mathrm{s}$ frequencies that are mounted on a rotable tower so that each beam scans simultaneously the same part of the sky, the 106.Mc/s echoes are found to occur most frequently within the auroral zone and the $56 \mathrm{Mc} / \mathrm{s}$ echoes some distance to the south. Relative frequencies and diurnal variations of the two groups of echoes, as well as their heights and geographical distributions, indicate that echoes originate from a threshold process. It is suggested that the echoes arise by critical reflection from centers of high electron density ( $1.4 \times 10^{8}$ and $4 \times 10^{7}$ electrons per $\mathrm{cm}^{3}$, respectively) that are formed close to but above the levels where the electron collision frequency exceeds the gyrofrequency of the elec. trons. (Item 5C-237, Met. Abs.)--Authors' abstract.

C.68 Currie, B. W. , Progress report of atmospheric ionization in Canada 1948-1951. I. U. G. G. Association of Terrestrial Magnetism and Electricity, Bulletin, No. 14, Transactions of the Brussels Meeting, :90-91, 1954.-.This report covers primarily activities in Canada that come to the attention of the Sub-Committee on Atmospheric Ionization of the Associate Committee of the National Research Council of Canada on Geodesy and Geophysics. As such, it concerns itself with researches in atmospheric electricity, aurora and geomagnetic phenomena in so far as they indicate the ionic conditions of the atmosphere, ionospheric reflections of radio waves and meteor ionization. It does not concern itself with such matters as tropospheric propagation of radar waves, reflection of radar waves by precipitation, ozone content of the atmosphere and cosmic rays, each one of these fields of investigation falling more properly under the jurisdiction of other existing committees of the National Research Council.

C.69 Currie, B. W., IGY aurora and airglow program. Ciencia, Mexico City, 16(11/12):309-316, 1956. 5 tables. DWB.. Special problems and techniques to be used during IGY in the auroral and airglow program, a list of the 100 Western Hemisphere stations from pole to pole making or intended to make visual, photographic and photoelectric observations of auroras, a list of 23 stations to make patrol-type spectrographic observations, 12 stations to operate high resolution spectrographs 
and photoelectric scanning spectrometers during IGY, 13 stations to make auroral radar studies and 10 stations to make airglow observations, and a detailed account of the Canadian auroral program are included in this informative article. (Item 9.7-35, Met. Abs.)--M. R.

C-70 Davidson, D., Reflexion of high frequencies during auroral activity. Nature, London, 167(4242):277-278, Feb. 17, 1@51. 2 figs., 2 refs. DWB-.Ionosphere reflection of $17.31 \mathrm{Mc} / \mathrm{sec}$ waves observed at Concord, Mass. on Aug. 8, 1950 during strong aurora. (Item 2.7-193, Met. Abs.)

C.71 De Kock, A. C., Het Noorderlicht. (Aurora borealis.) Hemel en Dampkring, 30ิ(3):104-108, March 1538. plate (6 auroral photos). "DWB--The theories of HULBURT, BIRKELAND, VEGARD and STORMER on the origin of the aurora, conclusions as to the composition of the high atmosphere obtained from auroral spectra, laboratory models showing auroral rings and torus space, and the disturbance to radio communication caused by the great aurora of Jan. 25-26, 1938 are mentioned in this review article. (Item 5C-87, Met. Abs.)-.-M. R.

C.72 Dieminger, Walter, Die Ionosphyre und ihr Einfluss auf die Ausbreitung elektrischer Wellen. (The ionosphere and its influence upon the propagation of radio waves.) Ergebnisse der Exakten Naturwissenschaften, Berlin, Vol. 17:282-324, 1938. 31 figs., 153 refs., 20 eqs. .-The purpose of this paper is to clarify the relationships between the ionosphere and radio wave propagation, specifically with regard to choice of frequencies under a variety of atmospheric conditions, times and seasons, as well as distances of path of communication. The introduction deals with the method of change of frequency and the echo method, followed by the discussion of (1) the theory of the ionosphere and (2) ionospheric observations under which subject headings the pertinent aspects are treated individually. The behavior of radio waves as influenced by meteors and aurorae is included. The bibliography envelopes the period 1925-1936...W. N.

C.73 Dieminger, Walter and Plendl, H., Abnormale Erscheinung in der Ionosphäre während des Nordlichtes vom 24. -25. Febr. 1939. (A bnormal phenomena in the ionosphere during the aurora of Feb. 24-25, 1939.) Beitrăge zur Geophysik, 55(2):189. 192, 1939. 3 figs., ref. DLC..Photographs of $80 \mathrm{~m}$ wave reflection during an unusual aurora, presented for the surface wave and the different $F$ layers, show distinct disturbances and a general increase of the reflecting light. (Item 5C-ৎ8, Met. Abs. )...A. A. 
C.74 Dieminger, Walter, Neue Ergebnisse der Ionosphärenforschung. (Recent result of ionospheric research.) Die Umschau, 44(3):37-40, Jan. 21, 1940. 8 figs. .-Measured virtual height versus frequency during times of magnetic storm and polar light. Describes normal characteristics of $E$ and $F$ layers. Points out geographical study of F2 and shows it behaves as if there were two ionizing components, one seasonal, and the other annual. The annual component is not yet found. (Annual has maximum simultaneously in both hemispheres, seasonal does not.) Des. cribes abnormal $\mathrm{E}$ reflections, storms. --L. A. Manning.

C-75 Dieminger, Walter, Zwei Arten der abnormalen E-Schicht. (Two kinds of E layer.) Die Naturwissenschaften, 33(5):154, Sept. 1946. 6 figs., 2 refs. .-Discusses the difference between the types of sporadic $E$ ionization found at medium and high latitudes. Compares curves showing the percentage of time for which sporadic $\mathrm{E}$ appears above $4 \mathrm{Mc} / \mathrm{s}$ at Tromsö, Oslo, and Kochel. Troms"̈ shows a maximum frequency of occurrence at night, the others near noon. Shows monthly dis tribution of occurrence. Tromsö, the high latitude station, shows little seasonal change, while Oslo and Kochel show a pronounced peak in occurrence in the summer, a minimum near December. He suggests that two causative mechanisms appear to be active in producing the two types of sporadic $E$. That active in the north might be called "Nordlicht.E". -.L. A. M.

C-76 Dostal, E. , Betrachtungen zur Erklärung des Weltraumechos des Polarlichtes und der magnetischen Störungen. (Explanatory notes on the worldwide auroral echoes and the magnetic disturbances.) Annalen der Physik, Leipzig, 5(14):971-984, Oct. 1932. 7 figs., 7 refs. , 3 eqs. ..The present theoretical attempts to explain the phenomenon are summarized, followed by a brief discussion in terms of the geometric optics and the physical nature of the reflecting "wall". .-W. N.

C.77 Dowden, R. L. (Commonwealth Ionos. Prediction Serv., Hobart, Tasmania), Low frequency ( $100 \mathrm{kc} / \mathrm{s}$ ) radio noise from the aurora. Nature, London, 184(4689), Supp. No. 11: 303, Sept. 12, 1959. 7 refs. DWB-.A report of "hiss" observations carried out at Hobart, Tasmania. Five frequency chan. nels were operated simultaneously, covering the gap from the normal "hiss" frequencies to the controversial $100 \mathrm{kc} / \mathrm{s}$ band. The center frequencies of these channels were $4.6, \subseteq .6,27$, 70 and $180 \mathrm{kc} / \mathrm{s}$. The observations establish that "hiss" can sometimes occur at frequencies up to $180 \mathrm{kc} / \mathrm{s}$, at least, suggesting that extraterrestrial noise much below a megacycle might be a "hiss" rather than "cosmic noise". (Item 11. 10. 157, Met. Abs.).-.I. S. 
C.78 Dowden, R. L. (Ionospheric Prediction Service, Hobart, Tas. mania), Ionospheric thermal radiation at radio frequencies in the auroral zone. Journal of Atmospheric and Terrestrial Physics, N. Y., 18(1):8-10, April 1960. 6 figs., 2 tables, 11 refs. DLC--Measurements of the temperature of the ionospheric D-region in the auroral zone from observations of 2 $\mathrm{Mc} / \mathrm{s}$ radio noise are described and compared with measure ments similarly made in the temperate region. The tempera. tures of the undisturbed and disturbed ionospheres at the two latitudes are found to be essentially similar, but the disturbing influence in the auroral zone is probably corpuscular rather than ultraviolet radiation. (Item 11. 10-324, Met. Abs.) -.Author's abstract.

C.79 Driatskii, V. M. and Besprozvannaia, A. S., Ionospheric conditions in the circumpolar region. Annales de Geophysique, Paris, 14(4):438-455, Oct./Dec. 1858. 13 figs., 11 photos, 3 refs. DLC.-Ionospheric observations from the drifting station SP-3 during the period May 15, 1954 to April 14, 1955, and especially observations made during the Arctic night, are given. The authors give a short description of the characteristics of the apparatus and of the magnetic field state. The results of the observations are given for the normal E layer, the $\mathrm{E}$ sporadic ionization, the $\mathrm{E} 2$ sporadic ionization, the normal F 1 and $F 2$ layers and the anomalous absorption of radio waves. Of greatest interest are the results received under the conditions of the Arctic night in connection with the F2 layer. The critical frequency for F2 layer during this period was unexpectedly high, reaching $5.6 \mathrm{mc}$ at times. The evident sporadic type of the layer being expressed in the sharp change of the critical frequency in the layer from hour to hour and from day to day, a definite connection with the state of the magnetic field proves its corpuscular nature. At the same time, the distinct shape of height-frequency dia. grams, normal for reflections from the F2 layer, stipulated by a wave radiation, can hardly be taken to agree with the corpuscular conception. It is still impossible to solve the prob. lem of the nature of observed high ionization in the F2 layer during a Polar night. (Item 11.1-53, Met. Abs.).-. A. V.

C-80 Dincan, R. A. and Ellis, G. R., Simultaneous occurrence of sub-visual aurorae and radio noise bursts on $4.6 \mathrm{kc} / \mathrm{s}$. Nature, London, 183(4675):1618-1619, June 6, 1959. 3 figs., table, 3 refs. DLC.-Observations at Camden, near Sydney (geom. lat. $42^{\circ} \mathrm{S}$ ) of enhanced emission toward the south of the red oxygen ( $6300 \mathrm{~A}$ ) airglow are interpreted as sub-visual aurorae. Simultaneous radio noise at $4.6 \mathrm{kc} / \mathrm{s}$ shows a definite correlation, both related to magnetic disturbance and tending to occur when the disturbance index $(K)>5$. The one can occur without the other and is explained. (Item 11.10-168, Met. Abs.)...W. N. 
C-81 Dyce, Rolf Buchanan, Auroral echoes of fifty megacycles obtained at College, Alaska. American Geophysical Union, Transactions, 35(2):375, A pril 1954. ..-CW and pulse methods were used to study returns from the aurora at $50 \mathrm{mc} / \mathrm{sec}$ during the summer of 1953, the equipment being located near the center of the auroral zone. Aurora was frequently seen in all parts of the sky but the VHF echoes were obtained only from azimuth angles within $60^{\circ}$ of magnetic north and with ranges of from $200-900 \mathrm{~km}$ but chiefly $400-700 \mathrm{~km}$. A verticallypointing antenna was used in an attempt to obtain echoes from overhead visible aurora but results were negative. Most of the echoes were therefore originating far north of the zone of maximum auroral activity. These data support the view that strongest echoes will be obtained when looking roughly perpendicular to the Earth's magnetic field. CW signal-strength recordings with antenna directed northward yield a diurnal variation of auroral propagation similar to results obtained at Ithaca, N. Y.

C.82 Dyce, Rolf Buchanan, Communication aspects of V.H.F. auroral reflections. Comell Univ. School of Electrical Engineering, Ithaca, N. Y., Technical Report 23, June 1, 1955. Unchecked. Microfilm available. DLC (AC 1, Publication No. 15, 011).-Experiments on frequencies around $50 \mathrm{Mc} / \mathrm{s}$ in northeastern United States and northern Alaska show the following: (I) The occurrence of auroral reflections have dis. tinct diurnal, seasonal, and solar activity variations; (2) the reflection condition is that the transmitted and reflected rays should be nearly perpendicular to the magnetic field at the position of the aurora; and (3) the fraction of incident energy reflected is on the order of 10.4. The effects of auroral reflections on VHF communication circuits are discussed... Author's abstract.

C.83 Dyce, R. B., VHF auroral and sporadic E propagation from Cedar Rapids, Iowa, to Ithaca, N. Y. Institute of Radio Engineers, Transactions, on Antennas and Propagation, AP 3(2): 78-80, April 1955. 8 figs., 14 refs. DLC.-The results of a total of 13,595 hours of valid observations with a $50 \mathrm{Mc}$ trans. mitter from April 1952 to May 1954 are presented and discussed. A simple and effective technique for separation of sporadic $E$ and auroral ionization is described. The diurnal variation for each type was determined; one being a nighttime, the other a daytime phenomenon. The seasonal variation determined for each mode of propagation showed maximum spo. radic $E$ at solstices and auroral signal minimum. Successful correlations with (a) visual aurora occurrence and (b) ionos. pheric sounder data were made. .-W. N. 
C.84 Dyce, R. B. (Geophysical Inst., College, Alaska and Cornell Univ., Ithaca, N. Y.), Auroral echoes observed north of the auroral zone on $51.9 \mathrm{Mc} / \mathrm{sec}$. Journal of Geophysical Research, Wash., D. C. , 60(3):317-323, Sept. 1955. 3 figs., 9 refs. DLC.-During Nov. 1954, a simple radar system designed for observation of auroral echoes at $51.9 \mathrm{Mc} / \mathrm{sec}$, was operated at Point Barrow, Alaska. Because this location is north of the accepted maximum of the auroral zone, most of the visible aurora is seen to the south of the observing station. The radar used a continuously rotating antenna to see with equal sensitivity in all directions, but more than $90 \%$ of the echoes were obtained from directions north of east and west. Echoes were obtained only from 500 to $1100 \mathrm{~km}$. These effects are explained by the theory of MOORE (See ref. C-207 208) as enlarged by BOOKER, GARTLEIN, and NICHOLS (See ref. C-28), requiring near-perpendicularity of radio ray paths to the lines of the earth's magnetic field. During visible aurora, propagation at $51.7 \mathrm{Mc} / \mathrm{sec}$ was investigated over an $800 \mathrm{~km}$ path from College to Barrow, across the auroral zone. Bursts of signal due to meteor ionization were readily observel. Propagation associated with aurora was almost nonexistent, even with visible aurora at the mid-path. If the theory of auroral echoes of HARANG and LANDMARK (See ref. C-127) were true, auroral propagation should have been readily detected. (Item 7. 10-70, Met. Abs.)--Author's abstract.

C.85 Dyce, R. B.; Dolphin, L. T. et al. (all, Stanford Res. Inst., Calif.), Aurora-like radar echoes observed from $17^{\circ}$ latitude. Joumal of Geophysical Research, Wash., D. C., 64(11): 1815-1818, Nov. 1959. 2 figs., table, 8 refs. DLC.-Anomalous echoes are regularly observed by a shipborne radar located at Antigua, British West Indies. These echoes, observed at 32 and $140 \mathrm{Mc} / \mathrm{s}$, have many of the characteristics of echoes from the auroras observed in the Arctic, although visible auroras should not be observable at Antigua more frequently than once in 7 yrs. Similar observations at Stanford Univ. indicate a correlation with one kind of sporadic $\mathrm{E}$ ionization. (Item 11F-33, Met. Abs.)--Authors' abstract.

C-86 Eastwood, E. ; Isted, G. A. and Bell, J. D. , Radar echoes from the aurora at $1300 \mathrm{Mc} / \mathrm{s}$. Nature, London, 189(4759): 115-117, Jan. 14, 1961. 2 figs., 2 tables, 8 refs. DLC.. Experimental results of 2 years observations with a high power L-band radar operating at $23 \mathrm{~cm} \lambda$ are discussed. It is shown "that the departure from the $90^{\circ}$ reflexion condition is less than $\pm 1 / 20$, and that the region of the ionosphere where the auroral reflexions take place is of the order of $20 \mathrm{~km}$ thick". The mechanism responsible for the aurora at $1306 \mathrm{Mc}$ can. be explained by Booker's scattering theory. ..W. N. 
C-87 Eckersley, T. L., An investigation of short waves, Institution of Electrical Engineers, Proceedings, 67(392):992-1032, Aug. 1929. 30 figs., 4 tables, eqs. DLC--Long paper dealing mainly with scattering, etc. Computes shape of pulse transmitted after random (like gas molecule) scattering. Ana. lyzes effect of dispersion on pulse shape, and finds $f$ must be very near the critical value to produce appreciable pulse blurring. Quotes Chapman on time for diffusion: at $100 \mathrm{~km}$ mean free path is $12 \mathrm{~cm}$, so diffusion over 100 meters of electrons would take less than one second, but of positive ions about $27 \mathrm{~min}$. At $125 \mathrm{~km}, 11 \mathrm{sec}$. Gives much data on directions of arrival of signals. Discusses atmospheric "whistlers" heard when an audio amplifier is connected to an antenna. Shows them most frequent during magnetic storms. Attributes to dispersion of click resulting from sudden stopping of $\alpha$ particles in the atmosphere. Considers mechanical analogy. In discussion, J. E. Taylor wonders whether "whistlers" aren't due to meteors, especially as they are heard at night. Eckersley wondered, but could find no reason why they should be, thinks Störmer's theories explain charged particles on dark side of earth. --L. A. Manning.

C-88 Egan, R. D. and Peterson, A. M. , Auroral noise at H. F. Journal of Geophysical Research, 65(11):3830-3832, Nov. 1960. 2 figs., 13 refs. DLC.-A brief review of previous studies of auroral noise at HF is followed by a report that riometer records during the large magnetic storm of Nov. 27 . 28 , 1959, recorded intense aurorally associated absorption at Meanook, Alberta. On that night a low latitude red aurora was present. The 3-hr $\mathbb{K}$ pindex reached a maximum value of 8. There is a close correlation apparent between the noise peaks recorded at Stanford, California, and absorption peaks at Meanook. The records taken at Pullman, Wash., indicate that both absorption and noise are present, and consequently, only the net effect was measured. Possible mechanisms for the generation of auroral noise include radiation from accelerated electrons in the $V$ an Allen belt. Synchrotron radiation from trapped electrons in the outer $\mathrm{V}$ an Allen region that are mirrored at a lower height as a result of a magnetic disturbance is regarded as the most likely mechanism for the noise observed at Stanford. Reference is made to the work of Dyce and Nakada (1959), Schwinger (1949), Chivers and Wells (1959). The experiments (direct measurement of the flux density in the outer Van Allen belt as a function of energy) made by Walt Chase, Cladis, et al (1960) are described. Arnold Hoffman's and Winkler's (1950) work with Explorer VI data is reported. In summary it is suggested that auroral noise does not correlate with daily magnetic indices, although there is a general over-all storm period correlation. --E. Z. S. 
C.89 Egeland, Alv; Hultqvist, Bengt and Ortner, Johannes (all, Kiruna Geophys. Observ. Kiruna, Sweden), A day-time maximum of oblique auroral reflexions observed in the auroral zone. Nature, London, 185(4712):519, Feb. 20, 1960. fig. , 8 refs. DWB-.From four months records of aurorally propagated very high frequency signals taken in the auroral zone at Kiruna, a diurnal variation curve with two maxima has been found. One of these maxima is in day time between 1200 and 1600 M.E.T. The experimental points include all observed reflections. The day time echoes have the same signal characteristics observed as night echoes on the Esterline Angus records. The direction distribution for day and night maxima is approximately the same; this has been determined by means of rotary antennae. (Item 12A.63, Met. Abs.)--E. Z.S.

C-90 Ellis, G. R. , Low frequency radio emission from aurorae. Journal of Atmospheric and Terrestrial Physics, 10(5/6):302. 306, 1957. 2 figs., table, 11 refs., 2 eqs. DWB-.This paper discusses the possibility that Cerenkov radio emission by auroral particles approaching the earth may contribute to the level of the continuous component of atmospheric radio noise observ. ed at frequencies of hundreds of kilocycles per second. It is shown that, provided the Cerenkov process is valid at radio frequencies, the flux density of the radiation may be as high as $10^{-21} \mathrm{~W} \mathrm{~m}^{-2}(\mathrm{c} / \mathrm{s})^{-1}$ at these frequencies, well above the minimum observable using current techniques. The frequency range of the emission would extend from a few hundred $\mathrm{kc} / \mathrm{s}$ to low audio frequencies. (Item 8. 10-302, Met. Abs.)-.-Author's abstract.

C.91 Ellis, G. R. A. (Upper Atmosphere Section, CSIRO, Camden, N.S. W.), Low frequency electromagnetic radiation associated with magnetic disturbances, Planetary and Space Science, N. Y., 1(4):253-258, Sept. 1959. 5 figs., 2 tables, 13 refs., ecis. DWB, DLC--Continuous observations of the amplitude and spectrum of naturally occurring radiation in the band $2-40$ $\mathrm{kc} / \mathrm{s}$ were made during the period June to December 1958 near Sydney, Australia. A large number of isolated noise bursts lasting for some hours were detected. The intensity ranged from $6 \times 10^{-19}$ to $6 \times 10^{-17} \mathrm{~W} \mathrm{~m}^{-2}(\mathrm{c} / \mathrm{s})^{-1}$ at $4.6 \mathrm{Kc} / \mathrm{s}$. Three main types of bursts were identified and classified on basis of their spectra which usually extended from 3 to $5 \mathrm{Kc} / \mathrm{s}$ and 2 to $30 \mathrm{Kc} / \mathrm{s}$, respectively. Major bursts, which were always of the latter two types, were clearly associated with strong auroral and magnetic activity and some showed a reproducible sequence of amplitude variation lasting about $36 \mathrm{hrs}$. On three occasions, a detailed correspondence between intensity of the noise and of simultaneously occurring red oxygen air. glow was observed. Theories and origin of the noise are dis. cussed. (Item 11.9-134, Met. Abs.).-Author's abstract. 
C.92 Ellis, G. R. A.; Cartwright, D. G. and Groves, J. R. V., Spaced observations of radio noise from the outer atmosphere. Nature, London, 184(4696, Supp. No. 18):1391-1392, Oct.31, 1959. fig., ref. DWB--It was suggested by G. R. A. Ellis that during some types of radio noise storms in the Earth's outer atmosphere, the source of noise may remain in constant position in right ascension. In this case the arrival of the storm will be recorded at the same local time at places of different longitude rather than simultaneously. In a majority of the cases noise bursts were recorded simultaneously at Camden and Adelaid. A distinct time delay was observed only during a major geomagnetic storm and aurora. (Item 11.12321, Met. Ábs.)-.A.H.J.

C-93 Fanselau, Gerhard, Úber die St"̈rung der Ionosphäre vom 24 26 Januar 1949. (On the disturbance of the ionosphere from Jan. 24-26, 1949.) Zeitschrift fur Meteorologie, 3(4):100-110, April 1949. 6 figs. , 5 tables. DWB.-On Jan. 2lst two large spots approached central meridian of sun. A magnetic storm began on Jan. 24th and is described in detail. Reception of the $1,250 \mathrm{~m}$ space radio wave from $K$ alundborg showed great variations. 27-day recurrences are clearly shown in Potsdam magnetic character figures from December to March. Associated auroras on Jan. 24-26 are listed and described. (Item 1. 5-116, Met. Abs.)--C.E.P.B.

C.94 Fendler, Ernst, Die ionosphärisch bedingte Übertragung ultra. kurzer Wellen. (Ultra short wave transmission affected by ionospheric activity.) Hochfrequenztechnik und Elektroakustik, 56(2):41-47, 1940. 13 figs., 12 refs., eq. DLC.-The purpose was to determine whether, how, and at what time of day and seasons the ionospheric propagation of $<12 \mathrm{~m} \lambda$ would be most favorable, considering the dependency of solar activity. Propagation through the E layer during abnormal ionization, the relationships between aurora borealis and ultra short radio wave propagation are discussed with some remarks on transmission power and antennas. Peculiar propagation conditions occurred in winter during aurora borealis. -.W. N.

C-95 Ferrel, Perry, Jr., Aurora U.H.F. propagation. Radio, Santa Barbara, Calif., No. 256:20-22, 74, Feb. 1941. fig. DLC.The so-called "Aurora-dx" signals at 43.0 and $44.10 \mathrm{Mc}$ associated with bright aurora displays, ionospheric and magnetic disturbances are discussed. Auroral effects on FM reception, Doppler effect and periodicity are also considered. The disturbances were at minimum in June and December, maximum in March and September. More severe storms occur in the northern hemisphere when the moon is below the earth's equator. .-W. N. 
C.96 Fleischer, R. (Rensselaer Polytechnic Institute, Troy, N. Y.), Auroral absorption of $18 \mathrm{Mc} / \mathrm{s}$ cosmic radio waves on $\mathrm{Feb} .11$, 1958. Nature, London, 181(4616):1156, April 19, 1958. fig. DWB.-This preliminary report illustrates graphically the ap. pearance of the spectacular auroral display observed widely in the United States on the night of Feb. 11, 1958. Observa. tion of its effect on the $18 \mathrm{Mc} / \mathrm{s}$ cosmic noise from the sky was made at the Sampson Station (lat. $42^{\circ} 47^{\prime} \mathrm{N}$; long. $73^{\circ} 27^{\prime}$ W). A description of the method of obtaining such sudden absorptions of cosmic noise is recorded briefly in the Astron. Journ., 62:243, 1957. (Item 12B-184, Met. Abs.)..N. N.

C-97 Forsyth, P. A. ; Petrie, W. and Currie, B. W. , Auroral radiation in the 3000 megacycle region. Nature, London, 164 (4167):453, Sept. 10, 1949. DLC..Pulses of radiation of duration $1.5 \mu$ sec, arriving in a random manner in bursts of short duration ( $<1 \mathrm{sec}$ ) were observed visually on the indicator of a radar set with transmitter off. Auroral origin was confirmed by observations of 20 aurorae, the part of the display to which the receiving aerial pointed at any time being photographed. Some correlation between frequency of pulses and intensity and type of aurora was found.

C-98 Forsyth, P. A. ; Petrie, W. and Currie, B. W. , On the origin of ten centimeter radiation from the polar aurora. Canadian Journal of Research, Sec. A, 28(3):324.335, May. 1950. 7 tables, 25 refs., eqs. DLC.-Short-lived bursts of $10 \mathrm{~cm}$ radiation from auroral displays have been received by radar equipment. The sources of continuous radiation from a partially ionized medium are briefly discussed. From a knowledge of the constants of the equipment used it is deduced that the power density at the receiver is at least $2 \times 10-10$ watts per $\mathrm{m}^{2}$ and it seems that the most likely source of this radiation is a plasma oscillation of the ionized volume asso. ciated with the auroral display. If it is so, the electron den. sity in at least localized regions must be of the order 1011 per $\mathrm{cm}^{3}$.... Authors' abstract.

C-99 Forsyth, P. A. ; Petrie, W; Vawter, F. and Currie, B. W., Radar reflexions from auroras. Nature, London, 165(4197): 561-562, April 8, 1950. 7 refs., 2 eqs. DLC.-Report of in. vestigation at Saskatoon, Canada. Radar equipment operating at a frequency of $3000 \mathrm{Mc} / \mathrm{sec}$ detected no radio echoes from auroras but well-defined and persistent echoes appeared on the $106.5 \mathrm{Mc} / \mathrm{sec}$ equipment. It could not be determined if the reflection came from the lower, brighter regions of the display or from higher points. Offers an explanation for ab. sence of echoes on the $3000 \mathrm{Mc} / \mathrm{sec}$ frequency. (Item $4 \mathrm{~J}$. 187, Met. Abs.).-.M.L. R. 
C-100 Forsyth, P. A. (Physics Dept. Univ. of Saskatchewan, Saskatoon, Canada), Radio measurements and auroral electron densities. Joumal of Geophysical Research, 58(1):5366, March 1953. 3 figs., table, 13 refs., 15 eqs. DWB.Radar echoes from aurora have been recorded simultaneously at frequencies of 56 and $106.5 \mathrm{Mc} / \mathrm{sec}$. The ratio of the echo amplitudes at the two frequencies varies between wide limits. An attempt is made to reconcile the experimental evidence with (i) partial reflections from the surfaces of large ionized regions in the aurora, (ii) scattering from inhomogeneities in the auroral ionization, and (iii) critical reflections from small volumes of intense ionization. It is concluded that (iii) is responsible for the typical auroral echoes which are observed at Saskatoon, although (ii) may be responsible for echoes of small amplitude which are often observed in the early stages of an auroral display. If these conclusions are correct, the occasional existence is indicated of electron densities of $10^{8}$ per $\mathrm{cm}^{3}$ in small volumes of the aurora. (Item 4J.231, Met. Abs.)... Author's abstract.

C.101 Forsyth, P. A. (Univ. of Saskatchewan), Radio wave reflections from aurorae. U. S. Air Force. Cambridge Research Center, Geophysical Research Papers, No. 30:117. 135, July 1954. 11 figs. , 17 refs. MH-BH-.-Radio wave reflections from auroras were reported by a number of workers including HARANG and STOFF-REGEN, PIERCE, LOWELL, CLEGG and ELLYETT, and ASPINALL and HAWKINS. Such reflections have been interpreted by HERLOFSON (See ref. C-141) as being due to partial reflection of the radio waves at the boundaries of the auroral structures. According to this interpretation, the electron density within an auroral structure could be as low as $4 \times 10^{4} / \mathrm{cm}^{3}$. Radio observations of auroras have been made at Saskatoon, Saskatchewan, for the past two years, utilizing radar equipment op. erating at $106.5 \mathrm{Mc} / \mathrm{s}$. Due to the nature of the apparatus used, the reflections were restricted to auroral forms located from 600 to $1100 \mathrm{~km}$ distant and elevated less than $7.5^{\circ}$ above the horizon from the observing site. The auroral forms most frequently observed by the radar method were the rayed arcs and rayed bands. The auroral origin of the radio wave echoes was confirmed by comparing the radar records with photographs of the same region of the sky, both types of observations being made simultaneously. (Item 6.9.332, Met. Abs.).-From author's abstract. 
C.102 Forsyth, P. A. and Vogan, E. L., The frequency dependence of radio reflections from aurora. Journal of Atmospheric and Terrestrial Physics, 10(4):215-228, 1\$57. 6 figs., 10 refs. , 10 eqs. DWB -.Auroral radio reflections on 4 frequencies ( $32.50 \mathrm{Mc} / \mathrm{s}$ ) transmitted from Greenwood, Nova Scotia, were received at Ottawa, $860 \mathrm{~km}$ to west. Periods of enhanced signals on one or more frequencies were often associated with auroral activity. Results are compared with those expected with various reflection mechanisms. They point conclusively to small auroral scattering centers in which ionization is sufficient to cause complete reflection, but suggest that localized absorbing regions are associated with each auroral display. (Item 8.8-126, Met. Abs.).. C.E.P.B.

C-103 Forsyth, P. A. (Univ. Saskatchewan), On the geometry of radio reflections from aurora. Canadian Journal of Physics, Ottawa, 38(5):593-603, May 1960. 5 figs., 15 refs. DWB, DLC--By assuming that auroral radio reflections are produced by volume scattering in clouds of ionization having the same spatial configuration as the visible auroral structures, and by taking into account the radar pulse duration and antenna beam width, it is possible to predict the probability of echo occurrence as a function of range and azimuth. This echo distribution is quite similar to that observed experimentally even when "aspect sensitivity" of the individual scatterer is neglected. Unfortunately, the optical evidence is not sufficiently extensive to permit precise calculations to be made, nor the radio evidence to permit detailed comparisons, but previous estimates of the shape of the scattering structures that have been based on the azimuthal echo distribution without regard to the factors discussed here are likely to be seriously in error. (Item 11.10-170, Met. Abs.).-Author's abstract.

C-104 Forsyth, P.A.; Green, F.D. and Mah, W., The distribution of radio-aurora in central Canada. Canadian Journal of Physics, Ottawa, 38(6):770.778, June 1960. 5 figs., 2 tables, 10 refs. DWB, DLC.-Five bistatic v.h. f. radio systems were operated in central Canada during the IGY for the purpose of detecting auroral ionization. Consistent records were obtained for a period of 5 months and these records have now been analyzed. Two types of events were detected. The nighttime $(A)$ events occur most frequently in the auroral zone and characteristically are observed simultaneously at two points separated by about $300 \mathrm{~km}$. The daytime (S) events occur simultaneously over a much larger area. The time of maximum occurrence of $\bar{A}$ events becomes later with decreas. ing latitude whereas the reverse is true for $\mathrm{S}$ events. The 
variation with latitude of the occurrence of $\bar{A}$ events is similar to that of other auroral phenomena. (Item 12A-76, Met. Abs.).-.Authors' abstract.

C.105 France. Meteorologie Nationale and Societe Meteorologique de France, Bibliographie Meteorologique Internationale, Nouvelle Series, v. 1-7, 1933-1939; (Unnumbered), 1946.. Last issue received, 1952, Fasc. 1-2. v. 1-6, pub. 19341949 ; v. 7, n.d. ; issues for 1946-pub. 1946.. Title varies: v. 1-2, Bibliographie Internationale de Meteorologie Gen. erale; v. 3-Bibliographie Meteorologique Internationale. DLC, DWB--Classified and partially annotated (in French) bibliographies covering periodical literature from all over the world. The material is arranged according to the U.D.C. scheme for meteorology (551.5). An author index and a subject index (with corresponding U. D. C.) are included. References to aurora will be found under 551.594.5, 551.594.51, 551.594. 52 and 551.594.53. 1952 issued by WMO as its OMM, No. 17. (Item 5C-65, Met. Abs.)--M.L. R.

C-106 Fricker, S. J.; Ingalls, R.P.; Stone, M. L. and Wang, S. C. (all, Lincoln Lab., M.I.T.), UHF radar observations of aurora. Journal of Geophysical Research, Wash., D. C. , 62(4):527-546, Dec. 1957. 14 figs. , 2 tables, 6 refs. DLC.. Radar returns from aurora have been observed on a 412.85 $\mathrm{Mc} / \mathrm{s}$ pulsed bistatic radar, located at South Dartmouth, Mass. Results obtained during two observational periods, Sept. 19 to Oct. 6, 1956, and Nov. 29 to Dec. 21, 1956, are given in terms of occurrence and position data, together with typical returns obtained on $\mathbf{A}$-scope and range-time intensity modulated displays. Positions of observed returns are compared with positions computed on the basis of requiring beam perpendicularity to the earth's magnetic field. Indications are that returns may be obtained from regions which are $2^{\circ}$ to $3^{\circ}$ off perpendicular, and that returns at larger off-perpendicular angles may be limited by a height factor. The returns appear to be limited to the height range of 90 to $160 \mathrm{~km}$ approximately. (Item 9.7-100, Met. Abs.)--Authors' abstract.

C-107 Fulton, B.J.; Petrie, L.E. et al. (all, Defence Res. Board, Ottawa, Canada), Transient modes of high frequency radio wave propagation across the auroral zone. Journal of Atmos. pheric and Terrestrial Physics, London, 16(1/2):185-186, Oct. 1959. ref. DWB, DLC--Records of sweep frequency oblique incidence radio soundings from the observations between Winnipeg and Resolute Bay since Oct. 1958, as illus. trated on photographs, and indicating extra reflections, are analyzed. Their characteristics depend on the size and electron density of the irregularity and its position to the F layer. ... T. T. 
C-108 Gadsden, M. (D. P. L. Auroral Station, Awarua Radio, In. vercargill, N.Z.), Studies of the upper atmosphere from Invercargill, New Zealand, Pt. 2, Correlation of the radar echoes and magnetic activity. Annnales de Geophysique, Paris, 15(3):395-402, July/Sept. 1959. (For Pt. 1, see ref. C-273; for Pt. 3, see ref. 109). 4 figs., 2 tables, 4 refs. French and English summaries p. 395. DLC.-Six months' operation of a $55 \mathrm{Mc} / \mathrm{s}$ radar has shown echoes occurring in 545 cases out of a possible 1220 three-hour periods. Analysis of this data reveals that the probability of occurrence of echoes increases with increasing magnetic $\mathrm{K}$-index, and that this probability is closely related to the local $\mathrm{K}$-index rather than the planetary $\mathrm{K}$-index. Evidence is found for a "radar auroral zone" whose size increases with increasing magnetic activity. It is shown that radar echoes are observed in the daytime to a lesser extent than suggested by the diumal variation of local K-index. (Item 11.6-117, Met. Ảbs.)--A Author's abstract.

C-109 Gadsden, M. , Studies of the upper atmosphere from Invercargill, New Zealand, Pt. 3, Radar echoes and visual aurorae. Ānnales de Geophysique, Paris, 15(3):403-411, July/ Sept. 1959. (For Pt. I, see ref. C-273; for Pt. 2 see ref. C-108). 3 figs., 2 plates, 2 tables, 10 refs. DLC--Evidence is presented and discussed that leads to the conclusion that there is no direct connection between radar echoing regions observed at $55 \mathrm{Mc} / \mathrm{s}$, and visible auroral forms. It is illus trated by graphs that the diumal variation of the occurrence of an echo observed at any range presents a broad maximum at midnight, whereas the observed auroral probabilities show fairly large fluctuations from hour to hour. An indirect asso. ciation exists since both aurorae (all - and rayed aurorae) and radar echoes have a greater probability of occurrence as the $\mathrm{K}$-index increases. But it is also shown, and illustrated by comments of several authors, that for high levels of magnetic activity a one-to-one correspondence does not exist. Finally, the analysis of series of simultaneous photographs of rayed auroral forms and of the radar A-scope shows that radar echoes are not to be identified with the rayed arc. (Item 11.6-118, Met. Abs.)..A. V.

C-110 Gerson, N. C., Radio observations of the aurora on Nov. 19 , 1949. Nature, London, 167(4255):804-805, May 19, 1951. fig. , 3 refs. DLC.-The phenomenon which is termed "auroral interaction" generally describes the reception of radio signals at a given location from a northerly direction, even though the transmitting station may be to the east, west, or even south of the receiver. In many instances, the transmitting and receiving stations which are able to establish radio 
communication through auroral interaction are located geographically so that they cannot communicate with each other during normal ionospheric conditions. When visible aurora is present, these stations may establish contact with each other by means of auroral interaction only when the directional antennae at both locations are directed 'northwards'. It was assumed that the portion of an aurora which has the greatest light intensity was also that portion which allowed auroral interaction to take place. Experimental procedures are described and it is concluded that the experimental method may be employed for the detection of the aurora when visual observations are difficult because of bright moonlight, scattered light or general cloudiness. The antenna beam width employed by the amateurs is about $30.35^{\circ}$, making it difficult to determine accurately the azimuth of any particular radio ray received from the aurora; however, narrower beam widths undoubtedly could be employed to provide much greater accuracies. It would also be expected that analyses of the rates and character of the garbling and fading of the returned signals would provide valuable information on the mass and velocities of the bombarding particles...E.Z.S.

C.Ill Gerson, N. C. (Air Force Cambridge Research Center), The Colloguium on auroral physics. Franklin Institute, Philadelphia, Journal, 253(4):331-338, April 1952. DLC-.The colloquium was held at London, Ontario on July 27-28, 1951 under the joint sponsorship of the Geophysics Research Division of the Air Force Cambridge Research Center and the University of Westem Ontario. The author presents a concise but informative summary of the discussion of the four sessions. The first session, with B. FRIEDMAN as chairman, was on "Electromagnetics as applied to auroral problems" and covered the following topics: "(a) the passage of a neutral stream of ionized matter from the sun toward the earth; (b) the interaction of this neutral plasma with the geomagnetic field to cause a ring current (and the early and late phases of magnetic storms); and (c) the subsequent movement of charges along the magnetic lines of force toward the terrestrial atmosphere eventuating in the observed luminosities." The theories of CHAPMAN and FERRARO on auroras and magnetic storms and MARTYN's extension of these theories were reviewed and MALFOR.S' experiment on the terrella was described. R. W. B. PEARSE was moderator of the second session which discussed "Interpretation of auroral and airglow spectra". The molecular spectra and atomic lines possibly present in the auroral spectra were considered as they relate to the composition of the ionosphere $(80-400 \mathrm{~km})$ and mesosphere $(400-1000 \mathrm{~km})$. Conclusions are drawn as to the positive existence of certain bands and lines and doubtful existence of others. "Quantum 
mechanics as applied to auroral problems" was the subject of the third session presided over by TA-YOU.WU. "This meeting considered the vitally important but very much neglected field of cross sections for collision of atomic, molecular, and ionized particles found in the ionosphere and mesosphere (a) to each other and (b) to the auroral bombarding particles." The fourth session was under the chairmanship of D.R. Bates. "Laboratory investigations of mesospheric processes" was the topic. In this session various experiments which have been undertaken, or are planned, to study various processes in the ionosphere and mesosphere were explained. (Item 3.11-278, Met. Abs.)-.M.L. R.

C.112 Gerson, N. C. (U. S. A. F. Cambridge Res. Center, Geoph. Res. Directorate), A note on auroral interaction. Journal of Atmospheric and Terrestrial Physics, London, 4(1/2):81-82, 1953. DWB--Several explanations are possible for the modulation of radio frequencies when reflected from auroral curtains. The probability is that the phenomenon is caused by a Doppler effect. However, this effect would be 5 to 50 times as great as is actually observed, assuming ionospheric winds of $250 \mathrm{~km} / \mathrm{hr}$. More probable results could be produced by assuming that the reflections come from the sides, not the ends of the approaching stream of solar protons. A few quantitative values for the models are given in tables. (Item 7.9. 266, Met. Abs.)--M. R.

C-113 Gerson, N. C., Radio observations of the aurora. Journal of Atmospheric and Terrestrial Physics, 6(5):262-267, May 1955. fig. , table, 6 refs. DWB--Radio wave probings mostly on $50 \mathrm{Mc} / \mathrm{s}$ North America showing auroral interaction in 1949-51 are listed. Monthly variation shows maximum frequency in April and Sept. -Oct. (Item 6.9-333, Met. Abs.).C.E.P.B.

C.114 Gerson, N. C., Diumal variation in auroral activity. Physical Society of London, Proceedings, Ser. B, 68(7):408-414, July 1, 1955. 3 figs., 19 refs. DWB..The variation deduced from analysis of radio contacts between amateurs in North America $\left(50-60^{\circ}\right.$ geomagnetic $\left.N\right)$ in $1949-51$ by waves incident obliquely to the ionized aurora showed a strong maximum at $21 \mathrm{~h}$ 75th W meridian time, about the time of greatest auroral activity; $86 \%$ of the contacts fell between 17 and $24 \mathrm{~h}$. The period of contact was longest in April, May and Oct. It does not seem to depend on geomagnetic control. (Item 7.2. 283, Met. Abs.)--C. E. P. B. 
C-115 Gotz, Friedrich Wilhelm Paul and Penndorf, Rudolf, Das Nordlicht vom 24./25. Februar 1939 in Arosa. (Aurora of Feb. 24.25, 1939, in Arosa.) Naturwissenschaften, 27(15): 241-243, April 14, 1939. 3 figs. DLC..A carefully detailed account of the conditions accompanying a brilliant auroral display which reached its greatest intensity at $11: 28 \mathrm{p} . \mathrm{m}$. on Feb. 24, and which reached $20^{\circ}$ above the $N$ horizon at $12: 40$ a.m. Feb. 25, just prior to the taking of the two vivid photos which are presented. A chart shows the course of the 3 magnetic elements at Collm Observatory, Leipzig, for the night in question, with arrows indicating times of greatest auroral activity so they may be correlated with the evident magnetic activity. The display was not visible in Norway but a magnetic disturbance ( 150 milliamperes) prevented telegraph communication between Norway and the Continent. (Item 5C-99, Met. Abs.).-.M. R.

C-116 Greenhow, J.S. ; Neufeld, E. L. and Watkins, C.D. (all, Univ. of Manchester), The scattering of $36 \mathrm{Mc} / \mathrm{s}$ radio waves by weak auroral ionization. Joumal of Atmospheric and Terrestrial Physics, London, 18(2/3):174-180, June 1960. 3 figs., 10 refs., 2 eqs. DLC..Some observations of weak back-scatter echoes similar in type to the echoes associated with visual auroras are described. With an equipment of high sensitivity at a frequency of $36 \mathrm{Mc} / \mathrm{s}$, these echoes are present to the north of Jodrell Bank (lat. $53^{\circ} \mathrm{N}$, geographic; lat. $56^{\circ} \mathrm{N}$, geomagnetic), for up to $30 \%$ of the observing time. The irregularities in ionization, which are aligned along the earth's magnetic field, have a length scale of $160 \mathrm{~m}$ and a scattering polar diagram with a width of approximately $\pm 3^{\circ}$. The horizontal dimensions of the ionized regions are about $500 \mathrm{~km}$ along parallels of geomagnetic latitude, and $50 \mathrm{~km}$ at right angles to this direction. Echoes from horizontal layers of ionization, associated with the field aligned irregularities, are also observed. (Item 12A-91, Met. Ábs.).-. Authors' abstract.

C-117 Gusev, P. I., Poliamye siianiia i verkhnie sloi zemnoi atmosfery. (Auroras and the upper layers of the earth's atmosphere.) (In: Vsesoiuznaia Konferentsiia po Izucheniiu Stratosfery, Leningrad, 1934, Trudy, p. 283-289, 1935. 6 refs., eqs.) Not trans. into English. DLC.-An article re. viewing the progress in study of the height, structure and composition of the atmosphere from 80 to $1000 \mathrm{~km}$ which has resulted from studies on the physics of the aurora and auroral spectra beginning with BIRKELAND's (See refs. C-23, 24) famous demonstration of the analogy between the aurora and laboratory models using cathode rays, and his conclusions regarding the corpuscular radiations from the sun, and later 
work of VEGARD (See refs. C.276, 277) in identifying auroral spectra lines. Theory which allows calculation of temperature in auroral layers is presented. MARIS and HUL. BURT's UV theory of aurora is also reviewed critically. Ionization and radiowave reflection from ionosphere also considered. (Item 5C.68, Met. Abs.).-.M. R.

C.118 Gustafsson, Georg; Egeland, Alv and Aarons, Jules, Audiofrequency electromagnetic radiation in the auroral zone. Joumal of Geophysical Research, Wash., D. C., 65(9): 2749-2758, Sept. 1960. 9 figs., table, 18 refs. DLC.During three one-month periods, continuous spectograms of the electromagnetic energy in the spectral region between 10 cps and $10 \mathrm{kc} / \mathrm{sec}$ were recorded in Kiruna, Sweden. The records were examined from the viewpoint of background energy. Throughout the entire frequency band studied, there is a low daytime signal level and a nighttime maximum. The ratio of the maximum to minimum amplitude varies as a function of frequency (the higher maximum-to-minimum ratio occurs at the lower frequency range of 20 to $200 \mathrm{cps}$ ) and with the season of the year. It is concluded that the daytime ionosphere absorbs the energy throughout the entire spectrum studied. It was found that, although strong deviations of the signal level from the normal were often associated with geomagnetic disturbances, there was a general lack of correlation between magnetic index and low-frequency noise, except in the 10 to $45 \mathrm{cps}$ frequency range. The origin of the background signals is probably two-fold, atmospherics from great distances as well as magnetic and exospheric fluctuations contributing to the lower band. On eleven occasions, electromagnetic radiation associated with micro. pulsations of the earth's magnetic field was detected. Two frequency bands were identified: one centered at $750 \mathrm{cps}$, which is the gyro frequency for protons at an altitude of 100 $\mathrm{km}$ above Kiruna; and the other ranging between 1.8 and 4.5 $\mathrm{kc} / \mathrm{s}$, which has been identified as hiss. On all but one of the occasions when emissions were detected the $750 \mathrm{cps}$ signals were quite stable in frequency. During four of the longest periods when radiation was recorded, the low frequ. ency emissions were received between two phases of a mag. netic storm; micropulsations were simultaneously evident on the Kiruna magnetograms. (Item 11.12-324, Met. Ảbs.)... Authors' abstract.

C.119 Hagg, E. L. and Hanson, G. H. , Motion of clouds of abnormal ionization in the auroral and polar regions. Canadian Joumal of Physics, 32(12):790-798, Dec. 1954. 10 figs. , 3 refs. DLC-.Examination of records taken in rapid succession at ionospheric stations in Northern Canada has revealed 
three distinctive types of echoes corresponding to clouds of ionization in motion. One type is ascribed to clouds of spo. radic $E$ moving horizontally over the station. The second type is believed to be due to clouds of ionization descending vertically from the $F$ region to the E region, while the third type appears to be from clouds moving at extremely high velocities in the $E$ region. Reflections of the second and third types have been seen only at stations in or north of the auroral zone. The characteristics of the echoes, the velocities of the clouds, and other associated phenomena are discussed. .. Authors' abstract.

C-120 Hagg, E. L. ; Muldrew, D. and Warren, E. (all, Defence Res. Board, Shirley Bay, Ottawa), Spiral occurrence of sporadic E. Journal of Atmospheric and Terrestrial Physics, 14(3/4):345-347, June 1959. 2 figs., table, 5 refs. DWB, DLC.-High density sporadic $E$, as observed at high latitudes, is distributed in a spiral form very much like St'brmer's pre. cipitation spiral for negative particles shown in comparison with results obtained at 11 selected stations (1944-1955). Tabulated sporadic $E$ is here considered only in a single variety, namely when the vertical incidence reflection of a frequency $>5 \mathrm{Mc}$ occurred (or $7 \mathrm{Mc}$ for 3 stations). .. W. N.

C-121 Hakura, Yukio; Takenoshita, Yugoro, and Otsuki, Toshiharu (Hiraiso Radio Wave Obs., Radio Research Lab.), Polar blackouts associated with severe geomagnetic storms on Sept. 13, 1957 and Feb. 11, 1958. Japan. Science Council. Ionosphere Research Committee, Report of Ionosphere Research in Japan, 12(4):459-468, 1958. 7 figs., 18 refs. DWB, DLC.-Examining through the world-wide ionospheric disturbances on Sept. 13, 1957 and Feb. 11, 1958, outstanding features of polar blackouts associated with severe magnetic storms have been discovered. A distinct region of abnormal ionization (usually called polar blackouts) appears in the polar region well before (about 20 hours) the onset of the geomagnetic storms. Such an ionized region builds up in the polar cap by the time of the sudden commencement (SC) of magnetic storm, and then spreads toward lower latitudes as the geomagnetic storm develops. A rather spiral-like pattern of the abnormal ionizing region is formed during the main phase of magnetic storm. Individual world-wide patterns of abnormal ionization, which are deduced from $\mathrm{F}_{\min }$ data, are shown for the respective stages of magnetic storm. It is quite a remarkable fact that some corpuscular invasion exists in the polar cap well before the SC of magnetic storms without inducing any appreciable world-wide geomagnetic disturbance. Spiral precipitation of particles indicates a rather complicated mechanism of the corpuscular intrusion 
into the earth's upper atmosphere, suggesting the revival of the old Birkeland-St'ormer theory. Some discussions about these phenomena are also given. (Item 12.3-134, Met. Abs.) -.Authors' abstract.

C.122 Hakura, Yukio and Takenoshita, Yugoro (both, Hiraiso Radio Wave Obs., Radio Res. Labs.), On the short wave transmission disturbance of Feb. 11, 1958. Japan. Science Council. Ionosphere Research Committee, Report of Ionosphere Research in Japan, 12(1):10.15, 1958. 5 figs. , 3 refs. DWB, DLC..The storm was of unusually large scale and was accompanied with intense radio disturbance. Herein, the data obtained at Hiraiso Radio Wave Observatory are dis. cussed and graphically presented. $\bar{A}$ fading rate counter was also used to investigate the detailed characteristics of propagation conditions. The present discussion is confined to the changes of field intensity levels and fading rates of 4 circuits, WWV, SF, WWVH and JJY. Then locations are shown on a map. The main features discussed and graphically represented are: 1) a sudden change of fading rate in WWV immediately after the onset of magnetic storm, 2) a southward shift of the fluttering fading of radio waves during the course of a magnetic storm; 3) an unusual rise of nighttime field intensity levels and fading rates of JJY and WWVH which coincides with the appearance of auroral echoes in the ionospheric record of vertical incidence observations. (Item 12A.92, Met. Äbs.)..-I. S.

C-123 Harang, Leiv and Stoffregen, W. , Der Polarizationszustande der Radiowellen bei der Reflexion Schichten, die während erdmagnetischen Stôrungen und Nordlichter gebildet werden. (Polarization of radio waves reflected from the ionized re. gions formed during magnetic disturbances and auroras.) Hochfrequenztechnik und Elektroakustik, 53(6):181-187, June 1939. 11 figs., 16 refs., 5 eqs. DLC..Purpose of the experimental investigation discussed was to check the polarization of normal $E$ and $F$ echoes at vertical incidence. To this end, a $50 \mathrm{~kW}$ transmitter and with the receiver at $300 \mathrm{~m}$ distance were used at 3-8 $\mathrm{MgHz}$. Film strip recording shows that echoes from the $E$ layer, ionized during magnetic disturbances and aurora, were of the two circular component type, of which the predominant component in comparison with normal $\mathrm{E}$ echoes was the lesser absorbable one...W. N.

C.124 Harang, Leiv, Vertical movement of the air in the upper atmosphere. Joumal of Geophysical Research, 41(2):143-160, 1936. DLC--Considers auroral heights and radio echoes, and considers among other things three-fold splitting of traces. Shows pictures. --L. A. Manning. 
C-125 Harang, Leiv and Stoffregen, Willi, Echoversuche auf ultra. kurzwellen. (Echo experiments with USW.) Hochfrequenztechnik und Elektroakustik, 55(4):105-108, April 1940. 4 figs. , 4 refs., 3 eqs. DLC.-Experiments were conducted at Tromso Observatory using $\lambda 7.3 \mathrm{~m}$ with the purpose of investigating possible USW echoes from $60.100 \mathrm{~km}$ during a severe geomagnetic storm and aurorae. This was negative but reflections were obtained from an auroral display in zenith (equivalent reflection distance of $400-800 \mathrm{~km}$ ). Other experiments from the altitudes $8-20 \mathrm{~km}$ and at $17 \mathrm{~km}$ are also described. -. W. N.

C.126 Harang, Leiv, Experimental studies of the reflection of radio waves from the ionized regions. Geofysiske Publikasjoner, Oslo, 13(4); 1942. 24+ p. 28 figs., 2 tables, 23 refs., eqs. DLC.-Discusses observational results of: (1) the state of polarization of radio waves reflected from ionized layers formed during terrestrial magnetic storms and aurorae; (2) scattering of radio waves from great virtual distances; (3) scattered reflections from the niveau of the $E$ layer; and (4) reflections of ultrashort waves from the ionized regions. These studies were conducted during April, May, June and December 1938, and January 1939, at Troms"8 where the inclination of the earth's magnetic field $\varphi=69^{\circ} .7$ $\mathrm{N}, \lambda=18^{\circ} .9 \mathrm{E}$. Gr.) is about $77^{\circ}$. Instrumentation and tech. niques employed for each study are described in detail. (I) $\mathrm{E}$ and $\mathrm{F}$ layer echoes were studied during (a) normal, (b) small and (c) strong terrestrial storms. The transmitter and receiver operating at the frequency range $3.9 \mathrm{Mc}$ were interspaced by $300 \mathrm{~m}$. It was found that (a) the echoes largely consisted of the ordinary component; (b) frequently both components were distinctly reflected even on lower frequency, indicating steep gradient electron density at the lower E layer edge; and (c) when the echo was about to vanish on higher frequencies, only the extraordinary component was reflected. Hence, a quantitative estimation of maximum electron density of a layer produced during a storm is feasible. (2) $A$ high-power transmitter operating at 7-13 $\mathrm{Mc}$ enabled reflections equal to $500-2500 \mathrm{~km}$ virtual distances. Four different phases in diumal variation are tabulated. Scattering heights varied from $500-800 \mathrm{~km}$ down to $150-200 \mathrm{~km}$ during stronger perturba tions. (3) Since the observed "life-timed" echoes of $1 / 2$ to $2-3$ sec duration showed a frequency curve almost identical to Appleton and Piddington's from southeast England, and which involves the height interval at the lower boundaries of aurorae; careful observations at $200-400 \mathrm{~km}$ were made when the $E$ region echoes were most frequent. No scattered echoes were observed however. (4) On Dec. 16 during a violent mag. netic storm associated with intense (red) aurora in zenith, 
echoes on $7.3 \lambda$ were observed as irregular scattering from $400-800 \mathrm{~km}$. Simultaneous control at $1-15 \mathrm{Mc}$ gave no echoes implying a measure for upper limit of maximum value electron density of terrestrial magnetic storm and aurorae produced layers. Echoes from $8.20 \mathrm{~km}$ as received with adjusted equipment interspaced at 150,900 and $2000 \mathrm{~m}$ showed no fading indicating lateral rather than vertical deflections. It may well be that these echoes are explainable by local topographic scattering of the USW. -.W.N.

C-127 Harang, Leiv and Landmark, B., Radio echoes observed during aurorae and terrestrial magnetic storms using 35 and $74 \mathrm{Mc} / \mathrm{s}$ waves simultaneously. Joumal of Atmospheric and Terrestrial Physics, N. Y., 4(6):322-338, 1954. DLC. (See ref. C-200 and C-284)..-Observations have been made at Kjeller, Lillestrom and at Troms' (near the auroral zone) using pulse transmitters operating simultaneously on 35 and $74 \mathrm{Mc} / \mathrm{s}$. These transmitters were supplied from common pulse modulator and HT units and gave $25 \mathrm{kw}$ output into geometrically similar, rotatable, Yagi aerial systems. The echoes received were displayed on a twin beam oscilloscope with a common time sweep. The presence of echoes was closely connected with geomagnetic and auroral activity but there was no correlation between the position of the aurora in space and the range of the echoes. Further, when the aerials were pointed at intense auroral forms no echoes were received. Echoes could only be observed when the aerials were directed at a low angle of elevation toward the North. It is concluded that these echoes are not due to direct scat. ter from the ionosphere or from auroral structures, as assumed previously, but can only be explained as back scatter from land or sea after reflection at an intense $E_{S}$ layer formed along the auroral zone during aurorae and geomagnetic storms. An analysis of the characteristics of the echoes and the differences in the echo ranges on 35 and $74 \mathrm{Mc} / \mathrm{s}$ support this hypothesis. Different types of echoes are described and the results of polarization measurements summarized. Correla. tion in time between the echo amplitudes is studied by means of double pulse modulation, the time interval between the pulses being variable. Possible passive radiation from the aurora in the $10 \mathrm{~cm}$ band could not be detected with the equipment available. ...Authors' abstract.

C-128 Harang, L. and Tr'bim, J. (both, Div. Telecommunications, Kjeller, Lillestrom, Norway), Determination of the angle of arrival of auroral echoes. Journal of Atmospheric and Terres trial Physics, London, 14(1/2):107-110, April 1959. 3 figs., 5 refs. DLC.-The angle of arrival, $\theta$, of auroral echoes is measured by an interference method. The variation of $\theta$ with 
echo range $\mathrm{R}$ is demonstrated. $\theta$ varies from $15^{\circ}$ to $6.5^{\circ}$ when the range increases from 400 to $730 \mathrm{~km}$. The height of the reflection area must lie at $100-120 \mathrm{~km}$. (Item 11.9-125, Met. Abs.)--Authors' abstract.

C-129 Harrison, D. P. and Watkins, C. D. , A comparison of radio echoes from the aurora, Australis and Aurora Borealis. Nature, London, 182(4627):43-44, July 5, 1958. figs., 4 refs. DLC -.The Royal Society"s expedition to Halley Bay has provided an opportunity for a simultaneous study of the aurora australis and borealis by the radio echo technique, and a preliminary account of the results is given in this note. Full details of the equipment are given in the IGY Annals, 3, Pt. IV, 337 (1957) by Lovell, A. C.B. Preliminary results on the occurrence of auroral echoes for the period May-October 1957 have been compared with the Jodrell Bank results for the same period. Echoes from the aurora australis have been detected at Halley Bay on 134 out of 164 days of observation, whereas on only 13 days were echoes from the aurora borealis obtained at Jodrell Bank. A study of the echo geometry at both locations shows that the echoes are obtained from the region where the line of sight is normal to the local magnetic field at a height of about $110 \mathrm{~km}$ rather than from along a line of geomagnetic latitude as originally proposed by Bullough and Kaiser (1954). (See ref. C-43).--E. Z. S.

C-130 Harrison, D. P. (Jodrell Bank Experimental Station, Univ. of Manchester), Auroral radio echoes at Halley Bay. Royal Society of London, Proceedings, Ser. A, 256(1285):229-234, June 21, 1960. 5 figs. , 9 refs. DLC--The echoes observed are shown to fit closely the case of specular reflections from columns of ionization aligned along the local magnetic field at a height of about $100 \mathrm{~km}$. (IRE, Proceedings, Abs. No. 570).

C.131 Harrison, V. A. W. (DSIR, Radio Res. Sta., Slough, Bucks), An unusual ionospheric disturbance in the Antarctic on June 30 to July 1, 1957. Joumal of Atmospheric and Terrestrial Physics, N. Y., 18(1):72-75, April 1960. 2 figs. DLC.Recordings of changes in declination, $D$, of the earth's mag. netic field were made at Port Stanley and also at the FIDS magnetic observatory in the Argentine Islands a few miles from Port Lockroy, and it is therefore possible to compare the ionospheric observations obtained at the two stations with magnetic recordings made nearby. At Port Stanley the first phase of the storm was associated with the presence of scattered reflections from the $F$ region - there were no significant abnormalities in the $D$ layer of the ionosphere during the period. After 1900 UT on June 30 unprecedented 
departures from typical storm behavior were observed at both Port Stanley and Port Lockroy - the echoes showed unusually large spread. At Port Lockroy the F layer was so disturbed and spread echoes so intense that no estimate of critical frequencies or even of the ranges in which they might lie was possible. Conditions returned to normal by 1400 UT on July 1. -.E.Z.S.

C.132 Hartz, T.R.; Reid, G.C. and Vogan, E.L. (all, Radio Physics Lab., Def. Res. Board, Shirley Bay, Ottawa, Ont.), V.H.F. auroral noise. Canadian Journal of Physics, Ottawa, 34(7):728-729, July 1956. fig., 6 refs. DWB, DLC.-FOR. SYTH, PETRIE and CURRIE reported in 1949 (See ref. C-97) noting radio frequency radiation from the aurora, though later studies failed to support such occurrence. The present report shows radio frequency $(32 \mathrm{Mc})$ emissions at the time of an aurora on March 21-22, 1956. The fact that it was also noted on 50 and $53 \mathrm{Mc} / \mathrm{s}$ rules out the possibility that it was from a distant station. The signals might, however, originate from the disturbed ionosphere at the time of an aurora. On numerous other occasions similar signals or noise has been recorded at the Radio Physics Lab., Ottawa, at the time an aurora was observed visually and recorded on a magnetometer, and a photomultiplier directed at the northern sky. (Item 8. 10. 297, Met. Abs.)...M. R.

C-133 Hartz, T. R., A Auroral radiation at 500 Mc. Canadian Journal of Physics, Ottawa, 36(6):677-682, June 1958. 2 figs., 14 refs. DWB, DLC.-An observation of radio noise emissions at $500 \mathrm{Mc}$, from a type A red auroral display which occurred on Oct. 21-22, 1957, is reported. The circumstances surround. ing this phenomenon are considered, and the unusual display is linked to a large flare on the sun some $30 \mathrm{hrs}$ earlier. It is concluded that an unusually large particle flux for the ejected solar matter would produce sufficient ionization in local regions of the ionosphere so that auroral radio emissions would be possible. (Item 10.5-256, Met. Abs.)..Author's abstract.

C-134 Hawkins, G. S., Observations of the aurora borealis by radio methods at the Jodrell Bank Experimental Station of the University of Manchester. I. U. G. G. Association of Terrestrial Magnetism and Electricity, Brussels, 1951, Transactions, pub. 1954. p. 229-234. 6 refs. DWB.-The auroral displays, briefly reported in this note, were studied at Jodrell Bank by the reflection of radio energy from the associated regions of ioniza. tion. Five sets of apparatus were used each consisting of an aerial system, pulsed transmitter, and receiver. The apparatus details are tabulated. Results obtained with each set are 
discussed and graphically represented. Photographs of echoes showing diffuse and discrete aurora on different dates are reproduced. (Item 12A-100, Met. Äbs.)..I.S.

C-135 Helbronner, Paul, Sur l'aurore polaire du 3 septembre et sur son action dans les transmissions radiotélégraphiques. (The polar aurora of Sept. 3 and its effect on radio transmission.) Académie des Sciences, Paris, Comptes Rendus, 191(14): 536-538, Oct. 6, 1930. DLC.-Brief comments are given on the auroral influences on the radio links Paris - New York; Japan - France; Beyruth - Paris; Rio de Janeiro and Buenos Aires...W. N.

C-136 Hellgren, Gossta and Meos, Johan (Res. Lab. of Electronics, Chalmers Univ. of Techn., Gothenburg), Localization of au. rorae with $10 \mathrm{~m}$ high power radar technique, using a rotating antenna. Tellus, 4(3):249-261, Aug. 1952. 18 figs., 11 refs. DLC.-The paper describes the $10 \mathrm{~m}$ high power recorder with a rotating antenna that is used since May 1951 for the localization of aurorae at the Radio Wave Propagation Laboratory of the Kiruna Geophysical Observatory $\left(67^{\circ} .8 \mathrm{~N}, 20^{\circ} .5 \mathrm{E}\right)$. Continuous observations during the time of May 1951-March 1952 have disclosed periods of auroral activity. The preliminary results from these observations indicate that there is a good correlation between the auroral activity, the magnetic activity, and the appearance of the $\mathrm{N}_{1}$ layer, a special type of sporadic $E$ ionization often appearing in connection with magnetic bays and supposed to be caused by the same ionizing agent as the aurora. The distribution in range and bearing of the recorded aurorae agrees with the simple theory that most of the radio wave scattering comes from those points where the rader beam is perpendicular to the surface of the auroral discharges. The calculated height distributions of the reflection centers have maxima around $120 \mathrm{~km}$. (Item 4.6-247, Met. Abs.)--Authors' abstract.

C-137 Hellman, Gustav, Repertorium der deutschen Meteorologie. ( $A$ reportory of German meteorology.) Leipzig, Wilhelm Engelmann, 1883. 995 p. chart, table. DLC.-Approximately 60 references to works of German authors on Aurora and Auroral theories are listed on p. 687.688, 690. (Item 5C-255, Met. Abs.)--M.L. R.

C.138 Henderson, J. P., Aurora and radio. Royal Astronomical Society of Canada, Journal, 17(8/10):374-378, Nov./Dec. 1923. DLC--With the sharp electrical discharges, possibly also streamer aurorae, clicks are heard in radio receivers and no particular relation was found to exist regarding good or bad reception. Clicks and grinders are probably results 
of thunder storm conditions somewhere. With corona and brush discharges, also waving curtain aurorae, hisses are heard. It is likely that with aurorae like sounds might be produced but of such audibility outside of its wireless manifestations or its laboratory analogues there is still question. Good receiving conditions nearly always accompany arc and curtain aurorae. --Author's abstract.

C.139 Heppner, J.P.; Byme, E.C. and Belon, A.E. (Univ. of Alaska, College, Alaska), The association of absorption and Es ionization with aurora at high latitudes. Journal of Geophysical Research, 57(1):121-134, March 1952. of figs., 2 tables, 8 refs. .- Noctumal Es ionization and "no echo" occurrences, as recorded on H'f records, have been classified according to the absence or presence of aurora and to its form when present in the zenith areas. The analys is sup. ports the following generalizations: (1) Es ionization increases at successively greater heights as aurora approaches the College zenith from the north. (2) In the presence of different non-pulsating auroral forms the Es ionization varies with changes in auroral form in a manner similar to the change in luminosity. Likewise, variations in the height of maximum ionization parallel variations in auroral heights. (3) Complete absorption is only slightly more frequent during nonpulsating aurora than during absence of aurora but prevails in the presence of pulsating aurora. Geomagnetic relationships are discussed. (Item 4.2.286, Met. Abs.).-Authors' abstract.

C-140 Heppner, James, Association of absorption and sporadic E ionization with aurora at high latitudes. Alaskan Science Conference, 2nd, Mt. McKinley National Park, Sept. 4-8, 1951, Proceedings, p. 278.279, (pub. 1953?) DWB--Analysis of vertical incidence measurements of noctumal $\mathrm{E}$ ionization and absorption shows that: 1 ) when aurora is not present in evening, high frequency traces show $F$ layer but no $E$ layer. When aurora is present $E$ layer is always present. 2) $E_{S}$ ionization increases at successively greater heights as auroral arc approaches zenith from N. 3) Changes in height of maximum electron density parallel changes in auroral height. 4) Erratic radio transmission may be due to scattered reflec. tion from non-pulsating auroras, whereas absorption is more nearly related to pulsatory auroras. 5) Conditions associated with pulsatory auroras resemble those during magnetic bays. Correlations may differ with changes in geomagnetic latitude near auroral zone. (Item 5C-253, Met. Abs.)--M. R. 
C.14I Herlofson, N., Interpretation of radio echoes from Polar auroras. Nature, London, 160(4077):867.868, Dec. 30, 1947. 2 refs., 4 eqs. DLC..Quantitative consideration of the strength of the echoes observed by Lovell et al, indicates that the electron density must be $>4 \times 10^{4}$ and < 2 . $6 \times 10^{7}$ electrons $/ \mathrm{cm}^{3}$. The former figure postulates a thin reflecting layer with sharp boundaries, and even the latter figure indicates diffuse boundaries $<3 \mathrm{~m}$ thick. Since such boundaries could not persist as long as $20 \mathrm{~min}$, it is suggested that the observed echoes were reflected from the vertical surfaces of a stationary discharge (auroral arc) at a height of $200 \mathrm{~km}$ above the brightest area.

C.142 Hoffmeister, C. and Konig, Hermann, Nordlicht-Beobachtungen auf der Sternwarte Sonneberg der Deutschen Akademie der Wissenschaften. (Observations of auroras at the Sonneberg Observatory of the German Academy of Sciences.) Die Steme, Leipzig, 34(5/6):125-126, 1958. DLC.-Review of the observations of auroras during the night of March 1-2, 1957 at $23 \mathrm{~h} 40$ and during the night of June 30-July 1, 1957 at $23 \mathrm{~h}$. The first notice of increase of solar activity and visibility of the auroras was given by Moscow. The Gottingen Geophysical Institute registered a magnetic storm during the night of June 30-July 1, 1957. Transatlantic radiocommunications were interrupted. The aurora was also observed at Bonn University. (Item 11.2-297, Met. Abs.).-. A. V.

C-143 Institute of Radio Engineers, N. Y., Proceedings, Vol. 43, No. 11, Pt. 2, Nov. 1955, Index to abstracts and references 1946-1953. $189 \mathrm{pa}$ Author Index and Subject Index for each year, and List of Journals for years 1S48-1953. DLC.-Eight separate author indexes, 8 subject indexes and 5 journal indexes for the respective years are bound together in an excel. lently printed, 3 column to the page publication which, unfortunately, is almost illegible without a magnifying glass. The author indexes contain abbreviated titles and reference to abstracts. Subjects under which abstracts of interest to meteorologists may be found include absorption, anemometer, hot wire, atmosphere, troposphere, ionosphere, microwaves, radar, wave propagation, meteorology, atmospherics, aurora, cosmic radiation, meteors, geomagnetic storms, geomagnetism, astronomy and radio, refractive index, etc. (Item 7.11-60, Met. Abs. )..M. R. 
C-144 Isaev, S. I., Podgotovka $\mathrm{k}$ issledovaniiam poliarnykh siianii $\checkmark$ Murmanske. (Preparations for the study of auroras at Murmansk.) A kademiia Nauk SSSR. Mezhduvedomstvennyi Komitet po Provedeniiu Mezhdunarodnogo Geofizicheskogo Goda, Informatsionnyi Biulleten', No. 3:86-87, 1957. fig. DLC.: Thirty four stations situated in high geomagnetic latitudes of Antarctica, Arctic, Murmansk, Yakutsk, Cape Chelyuskin, etc., have been equipped with special (large) wide-angle photo cameras (C-180) for photographing auroras. One of these cameras installed at the Murmansk branch of the Scientific Research Institute of Terrestrial Magnetism, Ionosphere and Radio Wave Propagation of the Ministry of Communication U.S.S. R. (M. O.. NIZMIR) was tested in March 1957 at Murmansk. More than 3000 photographs of auroras of various intensity, cloud conditions and of other weather peculiarities were made. Films of auroras taken every minute and ionograms were obtained (latter was carried out at the automatic panoramic ionospheric station). A two month course for the training of observers for polar stations of the Northern Sea Route, attended by 20 students, took place in February - March 1957. (Item 11.2.298, Met. Abs.).. A. M.P.

C-145 Isaev, Sergei Ivanovich and Pushkov, N. V., Poliarnye siianiia, (Auroras.) Illus. by G. N. Gamon-Gamon. Mos. cow, Akkademiia Nauk SSSR, 1958. 111 p. 50 figs. , 14 plates, 5 tables. At head of t-p.: Akademiia Nauk SSSR, Nauchno-Populiarnaia Seriia. DLC (QC971. 178)--A popular (25, 000 copies) scientific, but very substantial and scholarly, monograph on the aurora in all of its geographic, esthetic and physicochemical aspects. The 12 separate chapters discuss in concise form, with effective illustrations or graphs (taken mainly from original Soviet sources), the history of study of the aurora, auroral charts, forms and classification, geo. graphic distribution, relation to magnetic storms, to solar phenomena, knowledge of composition and temperature of upper atmosphere from auroral data, radar location of auroras, spectroscopic and photometric studies, theories, effect on radio propagation and, finally, a chapter by G. N. GAMONGAMON entitled Moi zarisovki poliaenykh siianii ( $M y$ auroral sketches), gives the history of the excellent sketches of 14 auroral displays which are appended. These were made in 1937-38 at Tulom near Murmansk. The present work is mainly devoted to Russian investigations and workers from LOMONOSOV to IGY. (Item 11.5-9, Met. Abs.).-.M. R. 
C-146 Israẹl, Hans, Extraterrestrische Einflusse auf das luftelektrische Feld. (Extraterrestrial influence on the atmospheric field.) Germany. Deutscher Meteorologischer Dienst im Französischen Besatzungsgebiet, Wissenschaftliche Arbeiten, 1:62-07, 1947. 2 figs., table, 17 refs. In German; German and French summaries p. 67. DLC.-The four extraterrestrial causes of the atmospheric electric charges which vary from day to day are: 1) cosmic rays; 2) corpuscular radiation from the sun; 3) UV discharges from the sun; and 4) sunspot and related activity. Numerous attempts have been made since 1872 to show a quantitative relation between aurora and electric charge in the atmosphere. A chart (after J. SCHOLZ, 1935) shows a rise in the electric field, increase in current and conductivity about $15.20 \mathrm{~min}$ before an aurora and a sharp drop at onset to a minimum about 10 min after the onset of the aurora. Other authors' theoretical explanations of positive charge without aurora and negative or positive during aurora are presented. (Item 5.11.220, Met. Abs. )...M.R.

C-147 James, J.C.; Bird, L.E. ; Ingalls, R.P. et al., Observed characteristics of an ultra-high frequency signal traversing an auroral disturbance. Nature, London, 185(4712):510-512, Feb. 20, 1960. 3 figs. DWB.-An experimental study was made to determine the characteristics of ultra-high-frequency signals propagated through auroral type disturbances. The experiment was performed by illuminating the moon from a site in the auroral zone and receiving the reflected signals from the moon at two sites in mid-latitudes. Each site used an antenna having two orthogonal polarizations. It was found that an auroral disturbance in the path of propagation causes a rapid fluctuation in the polarization angle of the signal received, and an increase in the rate of fading of the signal received, but no measurable absorption of the signal itself. The observed rapid changes in polarization angle are probably due to changes of electron content of the ionosphere of the order of 10-17 electrons in a vertical column of I sq $\mathrm{m}$ cross section. (Item 12A-113, Met. Abs.)--E.Z.S.

C-148 Kaiser, T. R. and Bullough, K. (Jodrell Bank Exper. Stat., Univ, of Manchester Lr. Withington, Macclesfield, Cheshire, Eng.), Radio echoes from aurorae. Annales de Geophysique, Paris, 11(3):27@-283, July/Sept. 1S55. E figs., S refs. DLC--Radio echo auroral observations made at Jodrell Bank from 1950 to $1 \subseteq 53$ are analyzed. A systematic picture is obtained of the special distribution and motion of the reflective ionization. A close correlation of the results is found with visual auroral and magnetic data. (Iter 12A-116, Met. Abs. )--Authors' abstract. 
C-149 Kaiser, T. R. (Dept. of Physics, Univ. of Reading, Berks), Radio investigations of aurorae and related phenomena. (In: The airglow and the aurorae: a symposium, London, Pergamon, 1955. p. 155-173. \& figs., table, 41 refs., eqs.) DWB.-The paper discusses in some detail the characteristics of auroral ionization as revealed by metre wavelength radioe. sho observations in the auroral and subauroral regions. A close association between the radio echoes, visual auroras, magnetic activity, and radio star scintillations is revealed. A similar correspondence appears to exist between low frequency auroral type echoes and scintillations in the minauroral region, although these show some marked differences from the high-latitude phenomena. Some suggestions are made for the future lines of work, particularly during the International Geophysical Year, 1657-58. (Item 3.7-341, Met. Abs. )--Author's abstract.

C-150 Kaiser, T. R. (Dept. of Physics, Univ. of Sheffield 10, Eng.), The geometry of auroral ionization. Journal of Geophysical Research, Wash., D. C., 02(2):2@7-2SS, June 1957. 2 figs., 5 refs. DLC.-A note comparing the interpretation of results obtained by the author at Jodrell Bank in 1954, with the interpretations of PETERSON (1S55) at Stanford. It is explained that the pattern of radio echoes from auroral and auroral-type ionization agrees with the hypothesis that it is distributed along an arc which follors a parallel of magnetic latitude, rather than with the assumption of extreme specular reflection from narros columns of ioniza. tion aligned with the earth's magnetic lines of force. (Item 13.A-118, Met. Abs.)--E.Z.S.

C-151 Kaiser, T. R., Relationships betreen auroral ionization and magnetic disturbance. Annales de Geophysiçue, Paris, 17(1):76-7S, Jan./March 1CES. 3 figs., 7 refs. DLC-.This report presents results from a detailed analysis of the correlation between characteristics of auroral ionization and geomagnetic disturbances. The magnetic disturbance is associated with spatially localized regions of intense ionization moving horizontally along a magnetic parallel of latitude. It suggests that, while the ionized clouds must preserve nearly electrical neutrality, there is a net drift of negative charge in the direction of motion of the ionization. This is from the dark to the sunlight hemisphere and is thus opposite to that predicted by MARTYN in his extension of the CHAPMAN-FERRARO corpuscular stream theory. It also appears that, for a given magnitule of magnetic disturbance, the ionization during positive bays is more intense than during negative ones. This supports the view that the morning maximum may become relatively less pronounced at higher radio frequencies. (Item 10.1-35s, Met. Abs.).-... V. 
C-152 Kavadas, $\bar{A}$. and Glass, D. G. , Polarization of radar echoes from aurora. Canadian Journal of Physics, Ottawa, 37(6): 6ฐ0.697, June 1959. 6 figs., 5 refs. DWB, DLC.-Auroral ridar echoes at very high frequency received at antennas sensitive to linearly polarized components in directions symmetrical to the plane of polarization of the vertically polarized transmitted wave and the ground plane indicate that the received wave, in addition to an unpolarized component, contains a linear component of polarization tilted in the general direction of the earth's magnetic field lines. (Item 11.4-113, Met. Abs.).-.Authors' abstract.

C-153 Kenrick, G.W.; Braaten, A. M. and General, J., The relation between radio transmission path and magnetic storm eff $a$ cts. Institute of Radio Engineers, Proceedings, 26(7): 331-347, July IS3E. 12 figs. DLC.-This paper presents the results of a quantitative study of the relationship between the proximity of great circle transmission paths to the magnetic pole and of signal stability during terrestrial magnetic disturbances. Reception from Europe, as observed at Riverhead, Long Island, and San Juan, Puerto Rico, is compared during normal and disturbed periods. A brief description of the duplicate equipment and antenna systems employed at the tivo locations is included. ..E. Z.S.

C.154 Kim, J. S. and Currie, B. W. Horizontal movements of aurora. Canadian Journal of Physics, 35(2):160-170, Feb. 1SE6. 2 figs., 4 tables, 18 refs. DLC.-Measurements on the drifts of auroral forms at three stations to the south of the auroral zone in west-central Canada failed to show evidence of a motion due to the earth's rotation relative to fixed excitation pattern in space. The distribution and the magnitide of the speed of auroral structures parallel and normal to the geomagnetic meridians are substantially the same as for non-luminous ionic irregularities observed by radio methods. The speed of an auroral arc or band parallel to the geomagnetic meridian is apparently constant. Speed increases $\mathrm{with}$ geomagnetic activity, particularly in an east-vest direction. There is no indication of a reversal of the east-west motions close to local midnight. Speeds and directions show no characteristic variations with the time of night. -. Iuthors' abstract. (See ref. C- 167).

C-155 Kirby, S.S.; Smith, N. and Gilliland, T.R., The effects of ionospheric storms on radio transmission. International Scientific Radio Union, 5th Assembly, Venice and Rome, Sept. 1538, Proceedings, 5(1):206-23C. ref. DLC.-Describe ionosphere storm. In first, a turbulent phase, the ionosphere in auroral regions is literally torn to pieces. Then there is 
expansion and diffusion away from auroral zone. Effects are failure of high frequency communication, weakening of night-time broadcast sky wave, increase of daytime broadcast sky wave during first phase. During the second, decreased MUF, increased absorption at high frecuency, increased night time broadcast absorption. --L. A. Manning.

C-156 Knecht, R. W. (Nat'l. Bur. of Standards, Boulder, Colo.), Relationships between aurora and sporadic $E$ echoes at Barrow, Alaska. Journal of Geophysical Research, Wash., D. C., 61(1):59-69, March 1950. 3 figs., 16 refs. DLC.-During March 1951, a series of visual auroral observations was made simultaneously with ionospheric soundings at Barrow Alaska $\left(71^{\circ} \mathrm{N}, 156^{\circ} \mathrm{W}\right)$. Auroras were visible during $32 \%$ of the 379 observations, made at least every 15 min during the dark hours of 10 successive clear nights. Three nights are described in detail. Statistical results include (1) a strong tendency for sporadic $E$ (Es) echoes at frequencies $\geq 7 \mathrm{Mc}$ to be recorded when aurora was near the zenith; (2) a direct relationship between brightness of (inactive) aurora and the top frequency of Es echoes; (3) evidence for the correspondence of (oblique) Es echo ranges vith estimated slant ranges of visible auroral forms. The observations lend support to the view that ionization in the imme. diate vicinity of visible auroral forms gives rise to ionos. pheric type reflections at high frequencies. (Item 7. 0.267, Met. Abs. )..-Author's abstract.

C-157 Krüger, K. , Die drahtlose Nachrichtenübermittlung in den Polargebieten. (Radio transmission in polar regions.) Arktis, Gotha, 4(3/4):52-64, 1031. 4 figs., 10 refs. , 13 eqs. DLC.-The discussion is mainly concerned with the influence of the low sun and the neighboring magnetic pole on Arctic short wave communication. ..-W. N.

C-158 Landmark, Björn (Norwegian Defence Res. Establishment, Kjeller, Norway), Echoes from the lower ionosphere during Polar blackouts. Journal of Atmospheric and Terrestrial Physics, London, 12(1):79-30, 1953. 2 figs. DLC...This is a short summary report on the special pulse soundings carried out near the mid-point of a path in the period June 15 to July 31,1357 at Nordi $\left(65^{\circ} \mathrm{N}, 14^{\circ} \mathrm{E}\right)$. The note presents some results obtained at this station. They may have some bearings in connection with conditions of radio fade-outs in the Polar region. (Item 11E-33, Met. Abs.)..N. N. 
C-150 Lange-Hiesse, G., Das Internationale Geophysikalische Jahr, XII: Polarlicht und Nachthimmelsleuchten. (The I.G.Y. Pt. 7, Aurora and night sky light.) Umschau, 57(23):707709, Dec. 1, 1957. 4 figs., footnote refs. DLC--There is a permanent weak light source round the earth, and a much stronger occasional ring of light $22.24^{\circ}$ from the magnetic pole. This is the aurora. The cause of the night sky light is the ionization and dissociation of gases by UV sunlight at great heights, that of aurora corpuscular rays from the sin focused by the geomagnetic field. The In G. Y. program for world-wide study of aurora is set out, including tropics and radio waves, and especially German contributions to observation of aurora and night sky light. (Item 9.4-276, Met. Abs.).-C. E. P。B.

C-160 Lange-Hesse, Günther (Max-Planck Inst。f. Physil der Stratosphäre, Lindau kurzer und ultrakurzer Wellen an Polarlichtern. (Reflection of short and ultrashort waves from polar auroras.) Archiv der Elektrischen Ubertragung, Stuttgart, 11(0):253-231, June 1557. Pt. 2, Ibid., 11(7):233-203, July 10อ3. figs. , $\bigcirc 2$ refs. English summary p. 253. DLC--Recent progress achieved in different countries in observing and interpreting auroral echoes is reviewed. Sample radar scope photographs, and diagrams of echo distribution are presented. Applications to radio communication, navigation, and auroral observation are discussed. Of various interpretations proposed so far concerning different characteristics of auroral echoes, the a:thor favors those of BOOKER. (See ref. C-20), ..G.T.

C-161 Lamor, Joseph, Aurorae, electric echoes, magnetic storms. Nature, London, 133(3354):221-223, Feb. 10, 1534. 7 refs., eqs. DLC--Reference is made to the results obtained by $\triangle P P L E T O N$ and others regarding the complex connections be$t$ ween the optical and magnetic phenomena of the upper atmosphere, and consideration is given to electron densities required in the reflecting layer to provide reflection by rays following vertical or oblique paths. A scherne is next introdiced in which it is assumed that the aurora is due to lasting local pulsations on a large scale, of long period, excited by a local cause, large enough and of abrupt type, produced conceivably by arrest high up of an ionized torrent from outside sufficiently concentrated to require relief by propagation in waves. This could give the banded auroral cirtains with spiralling transmission along the magnetic field. The splitting by the earth's magnetic field of the $r$ diation into two cyclic components is examined for magnetic fields along and transverse to the waves and the methols by which these components could be verified. Finally, 
the effects of Hamiltonian group velocity and transition be$t$ raen energy levels are discussed. (Item 4J-Go, Met. Abs.) -.Science Abstracts, No. 1530, April 1034.

C-162 Lister, E。 $A_{0}$, Variationen der D-Schichtdämplung auf 245 $\mathrm{kHz}$. (Variations of D layer damping on $245 \mathrm{kHz}$.) Zeitschrift fur Meteorologie, 7(11):321-330, Nov. 1553. 13 figs., $1 \mathrm{refs}$. MH-BH.-Results of the measurement of reflection coefficient on $245 \mathrm{kHz}$ during midday and in the evening are communicated. The diurnal and auroral course of the damping is sho:vn, and the data with other frequencies compared. The damping values of the $D$ layer have a close relationship to atmospherics and a weak one to geomagnetic activity. $\mathbb{A}$ 24 -day recurrence tendency is clearly indicated. The midday vilues of the damping are 2 nepers in the winter and almost 7 nepers in the summer. (Item $0.1-121$, Met. Abs.)..A. A.

C.103 Law, P.G. and Burstall, T。, Macquarie Island. Australian National Antarctic Research Expeditions, Interim Reports, No. 14, Sept. 1556. $48 \mathrm{p}$. 11 plates, 5 tables, 70 refs. DWB (M0.3. 5 AC38i)..The general climatic features of the $\mathrm{i}$ sland are covered on $\mathrm{p}$. $20-35$ of this monograph. Observation and research work in geomagnetism, cosmic rays, aurora and radiophysics is covered on p. 30-43. Activities are illustrated and many references to works on the region appended. The history of the island, of the station on the island, and of work in each field of endeavor is outlined in detail in each separate chapter. (Item 12A-126, Met. Abs.) -.M. R.

C-154 Leadabrand, R.L. and Peterson, A.M., Radio echoes from auroral ionization detected at relatively low geomagnetic latitudes. Institute of Radio Engineers, Transactions, AP-O(1):05-7S, Jan. 1S58. 21 figs., 28 refs., 18 eqs. DLC.. This is an extension of an earlier paper (See ref. C-22S), describing the characteristics of auroral zone echoes at distance ranges between $1400 \mathrm{~km}$ and $4700 \mathrm{~km}$. The reflection mechanism is discussed in terms of a partially reflecting, semi-infinite ionized sheet. The identification of these highfrequency echoes at Stanford favors the hypothesis that they are reflections from the primary auroral particles. Curved e srth - curved ionosphere ray path derivations are appended. ..W. N.

C-105 Loadabrand, R.L. and Yabroff, I., The geometry of auroral communications. IRE Transactions on Antennas and Propagation, AP 6(1):30-87, Jan. 1558. DLC--The scientific knowledge and results obtained since $1 S 3 S$ when the radio amateurs discovered how to exploit auroral ionization for 
communication under otherwise difficult conditions are summed up here. Based upon analys is of accomplished geometrical studies and available material on radio amateur communication report, a geometrical study is presented which implies the feasibility of selected communication and forecast of the same. .-W.N.

C-166 Leadabrand, R.L.; Dolphin, L. and Peterson, A.M., Preliminary results of auroral echoes at College, Alaska. IRE Transactions on Antennas and Propagation, AP 7(2):127-136, April 1959. 35 figs., table, 12 refs., eq. DLC.-Auroral ionization at $398 \mathrm{Mc}$ was investigated with a SR I $400 \mathrm{Mc}$ radar located $100 \mathrm{~km}$ south of the maximum of the auroral zone. The two types of echoes observed, discrete and diffuse, are generally night and daytime auroral forms, the former visible. Using the auroral radar equation $P_{R} \propto P_{T} \lambda^{10}$ for comparison with earlier (negative) results at $210 \mathrm{Mc}$ and Canadian results at $488 \mathrm{Mc}$ the $\lambda$ dependence law for the reflection deduced $=P_{R} \propto P_{T} \quad 5$.
-.W.N.

C.167 Leadabrand, R.I.; Presnell, R.I. ; Berg, M. R. and Dyce, R.B. (all, Stanford Res. Inst., Menlo Park, Calif.), Doppler investigations of the radar aurora at $400 \mathrm{Mc}$. Journal of Geophysical Research, Wash., D.C., 64(9):1197-1203, Sept. 1959. 9 figs., 11 refs. DLC.-By means of a relatively sensitive $400 \mathrm{Mc}$ radar located at College, Alaska, the variation of Doppler shift of auroral echoes has been determined as a function of the following parameters: (1) a zimuth angle of the radar ray; (2) off-perpendicular intersection angle of the radar ray and the earth's magnetic field; (3) elevation angle of the radar ray; (4) range of the echoes; (5) altitude of the reflection centers; (6) time of day; (7) number of occurrences; (8) strength of the echoes. These data have been further delineated in terms of the type of echo seen (discrete or diffuse) and whether the data were taken before or after magnetic midnight. An estimate of the spectrum spread of auroral echoes has also been determined by pulse and by $\mathrm{CW}$ techniques. A consistent trend in these data has been found which would indicate an east-west motion of the ionospheric irregularities. There is no appreciable variation in the direction of motion with time of day or with respect to magnetic midnight. The mean velocity of the east-west motion appears to be $500 \mathrm{~m} / \mathrm{sec}$. These conclusions agree with those of KIM and CURRIE (1958) (See ref. C-154), but disagree with those of LYON and KAVADAS (1958) (See ref. C-178), NICHOLS (1957) (See ref. C-211), and BULLOUGH et al (1957) (See refs. C-43,44,45). (Item $11 \mathrm{H}-83$, Met. Abs.).-Authors' abstract. 
C-168 Leithaüser, G., Über ionosphärische Störungen und irdische Auswirkungen. (On ionospheric disturbances and their terrestrial effects.) Funk und Ton, Berlin, 3(3):127-143, 1949. 9 figs., 14 refs. -.-Describes normal daily variation of wireless echoes and its disturbances by Mogel-Dellinger solar effect, auroras and meteors, with illustrations. Most important are the new reflection levels due to "inbreak layers" associated with auroras and magnetic disturbances. These have marked effects on wireless transmission. There is also a relation between development of inbreak layers and of low pressure areas at the surface in the auroral region, illus. trated by charts Feb. 13-17, 1048 and meteorological observations Feb. 1-March 19. Association of Norwegian anti. cyclones and cold European winters with solar eruptions is also discussed, including possible ameliorating effect of atom bombs. (Item 3. 8-5, Met. Abs.)--C.E. P. B.

Leonard, Robert S., Radar echoes from the aurora. (In: Conference on Arctic Radio Wave Propagation, Alaska Univ., Jan. 1956, Papers. Issued May 1956. p. 58-71. 8 figs.) DWB.-Auroral radar work at Geophysical Institute is des. cribed. The major difference between the ordinary and the auroral radar is that the latter uses pulse lengths on the order of 100 microseconds to one millisecond and has much lower operative frequencies. Some auroral radar experiments obtain no echoes from aurora overhead despite visual aurora in the sky. Three different theories explaining auroral echoes are given. (Item 12A.13l, Met. A bs.).-A. H. K.

C.170 Leonard, Robert S., A low power VHF radar for auroral research. Institute of Radio Engineers, N. Y., Proceedings, 4.3(2):320.322, Feb. 1958. 3 figs. (incl. photos), 2 refs. DLC..A $5 \mathrm{kw}, 41 \mathrm{Mc}$ radar which was designed for use in the U.S. IGY program is described. The standard operating procedures are outlined and a sample of the records is shown. The design of a scaling machine is discussed and a method of utilizing the scaled data to produce an auroral echo activity index is described. (Item 11H-88, Met. Abs.)..Author's summary.

C.171 Lied, F. (Norwegian Defence Res. Establishment), Quantitative measurements of absorption in the auroral zone. (In: Polar Atmosphere Symposium, Oslo, July 2-8, 1956, Proceed. ings, Pt. 2, Ionospheric Section. N. Y., Pergamon Press, 1958. p. 135-146. 14 figs., 11 refs.) Also issued in Journal of Atmospheric and Terrestrial Physics, London, Special Supplement, Pt. 2, 1957, pub. 1953? p. 135-146. DLC.. In the study of "normal" auroral absorption as well as the disturbances, we need the application of different techniques with overlapping sensitivity since the observational 
phase in connection with ionospheric absorption is not terminated. The statistical distribution in time and space of the more catastrophic blackouts, a little of the real nature and cause of the general and excessive high absorption due to some ionizing mechanism, and the techniques for locating where in the ionosphere the excessive absorption takes place, as well as the magnitude of the extra ionization, have been covered in this paper. Techniques include the $\mathrm{CW}$ oblique measuring, the pulse amplitude, the Galactic, the cross modulation techniques, all of which must be used contemporaneously and forced to overlap as in the ionospheric recordings made in Canada, Alaska and Norway. (Item 10. $\odot-316$, Met. Abs.)--N. N.

C.172 Lindquist, Rune, Polar blackouts recorded at the Kiruna Observatory. Gothenburg, Sweden. Chalmers Tekniska Hogskola, Handlingar, No. 103, 1951. 24 p. photos., graphs, refs. Price: kroner 3. DWB.."Polar blackout" is the name $\mathrm{H}$. W. WELLS gave the phenomenon of fadeout that shows no connection with solar flares, contrary to the ordinary ones occurring in the lower latitudes. The phenomenon was studied by E. V. APPLETON (1932-1933) and by BRAMHOLT and SEATON (1941). In the present study at Kiruna $\left(67^{\circ} 50^{\prime} \mathrm{N}, 20^{\circ} 14.5^{\prime} \mathrm{E}\right.$ geomagnetic coordinates $65^{\circ} 1 \mathrm{~S}^{\prime} \mathrm{N}, 115^{\circ} 30^{\prime} \mathrm{E}$ ) a panoramic recorder (frequency range $1.16 \mathrm{Mc} / \mathrm{s}$ in $30 \mathrm{sec}$ ) and a recording magnetometer were used. The preliminary results indicate that an abnormally high absorption, caused by the impact of some ionizing agent (maximum probably occurs below the $100 \mathrm{~km}$ level), some sort of corpuscular bombardment--is the cause. (Item 3. 9-88, Met. Äbs. ).-W. N.

C-173 Lindquist, Rune A., A survey of recent ionospheric measurements at the Ionospheric and Radio Wave Propagation Observatory at Kiruna. Arkiv for Geofysik, Stockholm, 1(2-4):247-266, 1951. 28 figs., 10 refs. MH-BH.-Ionospheric research started at the Ionospheric and Radio Wave Propagation Observatory at Kiruna on Oct. 1, 1948. This report gives some preliminary results obtained from the recordings during the period October 1948-August 1849. Different types of sporadic $E$ echoes are classified and the diurnal and seasonal behavior of $E s$ is shown and discussed. The relation between $E s$ and magnetic disturbances is further discussed. A few typical polar blackouts are described and studied. The diumal and seasonal behavior of the ordinary layers is also shown as a final bi-product. (Item 4.3103, Met. Abs.)--Author's abstract. 
C.174 Little, C. G. (Asst. Dir., Geophysical Inst., Alaska), Radio wave propagation in the Arctic. Alaska. Univ. Geophysical Institute, Contract AF 19(604)-1089, Interim Scientific Report No. 1, Aug. 15, 1955. 74 p. 23 figs. (incl. photos), 3 tables, 23 refs. --An extensive study of radar echoes from auroras (on 12, 25, 50 and $100 \mathrm{mc}$ ), involving correlation with visual aurora (as shown by panoramic photographs made simultaneously at College, Alaska), shows that radio echoes probably come from ionized columns in the immediate vicinity of visible auroral forms, contrary to theory of HARANG and LANDMARK (1954) (See ref. C-127). Sixteen pairs of photographs presented in this report show good agreement as to direction and distance, and the few exceptions are explained as due to abnormally high visible or faint diffuse forms. Three theories concerning the mode of reflection from ionized auroral layers are examined and the one of R.K. MOORE (1952) (See ref. C-207, 208), involving right angle reflection from ionized columns oriented along magnetic lines of force, is shown to agree with actual observations of 311 auroral forms from Aug. -Oct. 1954. Greatest concentration is to be $\mathrm{N}$ by $\mathrm{NE}$ (geomagnetic) and about $600 \mathrm{~km}$ distant. None occur overhead and few to the S. Theoretical distribution chart gives nearly same pattern. Attempts at using longer waves (than $106 \mathrm{mc}$ ) have thus far been unsuccessful. Experiments on a microwave link $25 \mathrm{mi}$ long failed to show any meteorological influence (ducts). Most winter time ducts are too close to ground to be of any use with normal equipment which sticks up above the duct. An all-sky camera has been used for a year at College and for a shorter time at $\mathrm{Pt}$. Barrow; analysis of the $180^{\circ}$ bands show that these are very frequent (105 cases), with openings to $W$ just before midnight and to $E$ at 0300 being most frequent. Movements of bands to $E$ or $W$ were more random than theorized by MEINEL and SCHULTE (1953) who reported only westward drifts during evening and eastward during morning hours. $\bar{A}$ good correlation was found between intensity of auroral light and $E_{S}$ ionization. Whistlers were studied with special equipment and comparison will be made with those at lower latitudes (Corvallis, Ore. and Stanford) to see if dispersion is much greater in high latitudes as pre. dicted. Between July 1 and 10, 1955, 38 whistlers were recorded. Ábnormal TV signal reception and scatter was observed over high peaks of Alaska Range. (Items 9.8.29; 12A.132, Met. Abs. )..M. R. 
C.175 Little, C. G. ; Rayton, W. M. and Roof, R. B., Review of ionospheric effects at VHF and UHF. Institute of Radio Engineers, Proceedings, 44(8):092-1018, Aug. 1956. 2 tables, 182 refs., eqs. .-This paper summarizes the present day knowledge of ionospheric effects at VHF and UHF, with the exception of forward scattering of VHF radio waves by the ionosphere. The seven effects covered in the paper are: radar echoes from aurora; radar echoes from meteors; the Faraday effect and radar echoes from the moon; radio noise of auroral origin; absorption of radio waves by the ionos. phere; refraction of radio waves by the ionosphere; and the scintillation of the radio stars. A bibliography of 182 items is included. --E. Z. S.

C-176 Lovell, A.C.B., Clegg, J.A. and Ellyett, G. E., Radio echoes from the aurora borealis. Nature, London, 160(4063): 372, Sept. 13, 1947. 6 refs. Herl ofs on, N. , Interpretation of radio echoes from polar auroras. Ibid. , 160(4077):867868, Dec. 20, 1047. (See ref. C.141). DLC.- Echoes ob. tained during the night of $\bar{A} u g .15-16,1947$ apparently originated in a luminescent cloud which appeared near the end of an aurora streamer. HERLOFSON's article is a discussion of above cited paper. (Item 4J-169, Met. Abs.).-.M. R.

C-177 Lugeon, J., Les perturbations radiophoniques pendant l'aurore polaire du 25 Janvier, 1933, en Suisse. (Radiophonic perturbations during the polar aurora of Jan. 25, 1938, in Switzerland.) International Scientific Radio Union, 5th Assembly, Venice and Rome, Sept. 1938, Proceedings, 5(1): 326-327, DLC--Describes effects during the aurora. Great absorption was observed on signals from Holland and Germany. However, signals from the west remained audible, though weak. --L. A. Manning.

C-178 Lyon, G.F. and Kavadas, A., Horizontal motions in radar echoes from aurora. Canadian Journal of Physics, Ottawa, 36(12):1661-1671, Dec. 1958. 8 figs. (incl. photo), 18 refs. DWB, DLC.-A systematic motion of $48.2 \mathrm{Mc} / \mathrm{sec}$ echoes as. sociated with aurora is found at Saskatoon. The motion is toward the west before midnight and toward the east after midnight, the mean velocity in either direction showing a statistical relation to variations in the earth's magnetic field. No correlation is found between individual echo velocity and magnetic disturbance, and no period of zero velocity is observed. There is also evidence of an ordered relation between motion in the north-south direction and disturbances in the earth's magnetic field. (See ref. C-167). (Item IIH101, Met. Ábs.)--Authors' abstract. 
C.179 Lyon, G.F. (Univ. Saskatchewan), The association of visible auroral forms with radar echoes. Canadian Journal of Pnysics, Ottawa, 33(3):385-385, March 1560. 2 figs., table, 9 refs. DWB, DLC-.A peak in $43.2 \mathrm{Mc} / \mathrm{sec}$ echo occurrence is observed at Saskatoon corresponding in time to the period of breakup of quiet arcs into active rayed structures. This is also the time of most frequent occurrence of characteris tic "curl" forms in the aurora. If, as GARTLEIN suggests, the "curl " forms are formed by instabilities in a sheet beam then the primary particles are positively charged. (Item 12A134, Met. Abs.)--Author's abstract.

C-180 McInnes, B. , Auroral display observed from unusually low geomagnetic latitudes. Meteorological Magazine, London, 85(1018):114-117, April 1957. fig., 2 refs. DWB.-Observa. tions of aurora from ships in S. Indian Ocean (geomagnetic coordinates $43^{\circ} \mathrm{S} 184^{\circ} \mathrm{E}$ and $37^{\circ} \mathrm{S} 129^{\circ} \mathrm{E}$ ) on Sept. 8, 1956, are described. There was a world-wide magnetic storm, radio effects, etc. (Item 8.8-314, Met. Abs.).-C.E.P.B.

C-181 McInnes, B. and Robertson, K. A., Aurora, Marine Observer, London, 30(139):135-139, July 1960. fig. , tables. DWB, DLC--A log is given listing auroral observations for the period July-Sept. 1950 and some comments on the outstanding auroral displays. The auroras are classified and their geomagnetic latitudes and longitudes and inclinations are given. The most outstanding display was observed during July 15-13. This geomagnetic storm had a significant effect on some ships compasses and also affected radio reception. (Item 12A-137, Met. Abs.)--R.B.

C-132 McKinley, D.W.R. and Millman, Peter M., Long duration echoes from aurora, meteors, and ionospheric back-scatter. Canadian Journal of Physics, 31(2):171-181, Feb. 1953. 2 figs. , 6 tables, 10 refs. DLC--In the course of the Ottawa meteor program some unusual echoes have been detected on $33 \mathrm{Mc}$. Echoes from the aurora are discussed and correlated with visual observations. Two mechanisms of radio reflections from the aurora have been proposed but the data here presented are insufficient to favor one or the other. On Aug. 4, 1:48 six extremely long duration meteor echoes were observed. Since Aug. 1948, a weak semipermanent echo has been recorded, usually appearing at a range of about $80 \mathrm{~km}$, and enduring up to an hour. It is suggested that this echo is due to backscatter from the same sources in the lower Eregion that are presumed to be responsible for long range very high frequency propagation. (Item 4. 10-33, Met. Abs.) --Authors' abstract. 
C.183 McNamara, A. G. and Currie, B. W. (Physics Dept., Univ. of Saskatchewan, Saskatoon), Radio echoes during aurora. Journal of Geophysical Research, 55(2):279-285, June 1954. 4 figs. , 6 refs. DLC...A reassessment of all the Saskatoon data on 56. and $106 . \mathrm{Mc} / \mathrm{sec}$ echoes from aurora suggested that some of the $56 \mathrm{Mc} / \mathrm{sec}$ echoes might have occurred through back'ward scatter from the land via the lower part of the ionosphere. These could have originated only through the second lobe transmission of the radar equipment. Two antennae placed at heights to give the maximum resolution between echoes arising in directions corresponding to the first-and second-lobe signals were used to examine this possibility. The results show that practically all (if not all) the echoes were due to direct reflection from aurora. (Item 6. 2-39, Met. Abs.).-Authors' abstract.

C.184 McNamara, A. G. and Currie, B. W. , Polarization of radio echoes from aurorae. Nature, London, 174(4442):11531154, Dec. 18, 1954. 3 figs., 6 refs. DWB-.Observations at Saskatoon on $56 \mathrm{Mc} / \mathrm{s}$ are analyzed statistically for ratio (horizontal/vertical polarization) frequency variation as a function of range, showing a significant increase at 500-500 $\mathrm{km}$, and echo occurrence against radar range. Some observations on $106.5 \mathrm{Mc} / \mathrm{s}$ are included; these showed only plane polarization in sense of transmitted signals. (Item 6.6.330, Met. Abs. ).-C.E.P. B.

C-185 McNamara, A.G. (Saskatchewan Univ., Saskatoon, Canada), Double Doppler radar investigations of aurora. Journal of Geophysical Research, Wash., D.C., 60(3):257-269, Sept. 1955. 7 figs. (incl. photos), II refs. DLC..A pulsed double Doppler radar technique has been employed to study the 50.7 $\mathrm{Mc} / \mathrm{sec}$ signals reflected from auroral ionization. A spectrum analyzer was used in conjunction with the radar to measure the power spectra of the auroral echoes. The Doppler data are compared with observations of the visible aurora and with simultaneous echoes on 56 and $106 \mathrm{Mc} / \mathrm{sec}$ non-coherent high resolution radar equipment. An interpretation of the Doppler data is given, and several theoretical models of the reflection mechanism are examined in terms of their effect on the observed spectra. (Item 7. 10-71, Met. Abs.)--Author's abstract.

C-186 McNamara, A. G. , A continuously recording automatic auroral radar. Canadian Journal of Physics, Ottawa, 36(1):1-3, Jan. 1958. 6 figs., 6 refs. DWB, DLC--A simple, low power $50 \mathrm{Mc} / \mathrm{s}$ radar is described which has been designed for automatic recording of radio reflections from auroral ionization. The system features high sensitivity with good reliability. 
Photographic records are taken in the form of a continuous film strip displaying range and time. A complementary recording system employs circuitry which cancels interference and noise signals but produces an output signal proportional to the integrated echo intensity. The display in this latter case is in the form of a chart record from which data are immediately available. (Item 9.7-102, Met. Abs.)..Author's abstract.

C.187 McNamara, A. G. (Natl. Res. Council, Ottawa), An analysis of some statistical properties of auroral radar reflections and their relationships to the detection capabilities of the radar. Canadian Journal of Physics, Ottawa, 38(3):425-438, March 1960. 5 figs. , 2 tables, 6 refs., 22 figs. DWB, DLC -.A statistical model of auroral echo occurrence has been made from an analysis of observations at $48.5 \mathrm{Mc} / \mathrm{s}$ obtained over a number of years of continuous operation. The probability density distribution of auroral target cross sections $(\sigma)$ ias been examined experimentally, and the resulting curve fitted by simple mathematical relations. Both an inverse power law and an exponential law have been derived, of the forms $p(\sigma) d \sigma=k \sigma^{-} 1.67 d \sigma$ and $\left.p(\sigma) d \sigma=\sqrt{\sigma} \hat{\sigma} \hat{m}\right) e^{-\sigma / \sigma m} d \sigma$

These models have been interpreted in terms of distributed and localized targets, and used to analyze the echo occurrence indices and the effect which variation of radar parameters will have upon them. Both forms of the target law are useful although it is considered that the exponential form yields better agreement with observations over a wider range of the variables. (Item 12.4-356, Met. Abs.)-.Author's absiract.

C.188 McNamara, A. G. , Auroral radar observations at $48 \mathrm{Mc} / \mathrm{s}$ during the period of the Nov. 12, 1960, solar event. Canadian Journal of Physics, Ottawa, 3ৎ(4):625-627, April 1C61. fig. DWB, DLC--Simultaneous records from three auroral radars of similar characteristics have been analyzed for the period Nov. 11, to Nov. 16, 1660. The combined results show interesting patterns of activity. Short bursts of echoes are sometimes associated in time with sudden commencements. These echoes are not intense which may account for the fact that only the more favorably situated radars detect them. Evidence cited for a large PCA event suggests that at such times the strong low-level absorption significantly alters the detection capabilities of a VHF auroral radar, and may distort the apparent geographical distribution of the reflecting ionization. -.E. Z.S. 
C-189 Maehlum, Bernt (Norwegian Defence Res. Estab.), The diurnal variation of foF 2 near the auroral zone during mag.netic disturbances. Joumal of Atmospheric and Terrestrial Physics, London, 13(1/2):187-190, Dec. 1958. 3 figs., 7 refs. DLC.--During magnetic storms the critical frequency of the F2 layer usually decreases and this well known effect (APPLETON and INGRAM, 1935) has been the subject of numerous statistical studies. Distinguishing between disturbed and quiet days, the departure in the critical frequency, $\Delta$ foF2, due to magnetic storms can be studied more closely. It has been shown that the variation in $\Delta$ foF 2 can be separated into two components, a $S_{D}$ component which depends on local time and a $D_{\text {st }}$ component which depends on storm time (FUKUSHIMA, 1949). The aim of this note is to study the $S_{D}$ component for some stations in Scandinavia and Great Britain. It will be shown that the $S_{D}$ variation observed at Longyearbyen $\left(80^{\circ} \mathrm{N}\right)$ is similar to that observed at middle latitude stations, but the $S_{D}$ variation at Troms" $\left(70^{\circ} \mathrm{N}\right)$ is reversed. There is thus an analogy with the latitude effect of the geomagnetic storms, where the $S_{p}$ current systems are reversed within the auroral zone (FUKUSHIMA, 1953). Item 10.10-208, Met. Abs.).-Author's introduction.

C.190 Major, G., The association of pulsating and flaming auroras with complete ionospheric absorption at Macquarie Island. Australian Journal of Physics, Melbourne, 7(3):471-476, 1C54. 3 figs., 5 refs. DLC.-Simultaneous records show that pulsating or flaming auroras are frequently accompanied by complete absorption of waves incident vertically on the ionosphere, but the noctumal variations of frequency of occiurrence of the two phenomena are markedly different in form. --Author's abstract.

C-191 Martin, L. H. ; Helliwell, R. A. (both, Radioscience Lab., Stanford Univ., Calif.) and Marks, K. R. (U. S. Antarctic Res. Program, Wash. 25, D. C.), Association between auroras and very low frequency hiss observed at Byrd station, Antarctica. Nature, London, 187(4739):751-753, Aug. 27, 1960. 5 figs., 6 refs. DWB, DLC.-Observations during 1355 show a close association to exist between auroras and certain very low frequency hiss. The hiss usually occurs in a broad band with a center frequency of about $8 \mathrm{kc} / \mathrm{s}$. The intensity and band-width vary with ionospheric absorption and may also vary with the intensity of the auroras. The hiss drops to undetectable levels under conditions of extreme ionic absorption even in the presence of intense and active auroras. The center frequency of the hiss may be associated or related to the type of aurora. (Item 12A-151, Met. Abs.) --R. B. 
C-192 Martvel', F.E. and Pogorelov, V.I., O sviazi svetimosti poliarnykh siianii s radiolokatsionnymi otrazheniiami ot nikh. (The relation between the brightness of auroras and their radar reflections.) Akademiia Nauk SSSR, Izvestiia, Ser. Geofiz., No. 8:1052-1053, 1553. 7 figs. DLC. Transl. into English in the corresponding issue of the English language ed. of the Izvestiia, Bulletin of the Academy of Science of the USSR, Geophysics Ser. DWB--In order to determine the relationship between auroras and auroral radar reflections, the authors compare motion picture frames coinciding in time with radar signals. The material used was that obtained at Roshchino $\left(\varphi=60^{\circ} 12^{\prime} \mathrm{N} ; \lambda=20^{\circ} 34^{\prime} \mathrm{E} ; \Phi=56^{\circ} 35^{\prime}\right.$; $\left.\Delta=116^{\circ} 47^{\prime} \mathrm{E}\right)$. An analysis of the material indicates the following: (1) although it is impossible to determine the form of auroral illumination that is most effective from the point of view of radar reflection, many of the most intense reflections are close to the most pronounced radiation forms; (2) reflections with large amplitude correspond to the brightest reflection regions of the auroras in the supposed zones of reflection of the auroras, and no radar reflections were observed when the auroras were completely lacking; (3) during weak illumination radar reflections were rarely observed; (4) there were no instances in Roshchino when radar reflections preceded auroras; (5) there exist small differences (about $5-10^{\circ}$ ) between the azimuths of the most intense parts of the auroras in the supposed zones of reflection and the azimuths of the most intense radar reflections which, however, do not go beyond the limits of possible errors of observation and which may be explained by distortion of the trajectories of automagnetic waves in a medium of electronic inhomogeneities, which are considerable in the auroral regions; (6) the dimensions of the supposed zones of radar reflections are slight in comparison with the region of the heavens occupied by the auroras. (item 11H-106, Met. Abs.) --I. L. D.

C.193 Massey, H.S.W., The nature of the upper atmosphere. Endeavour, London, 13(50):81-85, April 1954. 4 figs., 6 refs. DLC--General but semi-technical treatment of structure and composition of the high atmosphere, the ionosphere, aurora and airglow according to latest results of rocket and radio research. (Item 6.4-117, Met. Abs.)--M.R.

C-194 Matsushita, S. (High Altitude Obs. of the Univ. of Colorado, Boulder), Some studies of the upper atmosphere in the auroral zone. Annales de Geophysicue, Paris, 14(4):483-491, Oct./ Dec. 1958. 3 figs., 28 refs. French and English summaries p. 483. DLC--Relations between ionospheric and geomagnetic phenomena in the auroral zone were studied from magnetograms both made at the same station by correlating striking features 
in each. When a bay disturbance of moderate range occurred at night, complete blanketing of $F 2$ by sporadic $E$ always happened at the time. When the range of bays was larger, blackouts usually occurred after the incidence of complete blanketing of F2. Slant $E_{S}$ occasionally appeared during bays, and it never occurred except during bays or bay-type variations during magnetic storms. Blackouts which occurred during daylight hours had no remarkable correlations with geomagnetic variations except during magnetic storms. In other words, there were two different types of the polar blackout; nighttime and daytime types. The height of the absorbing region responsible for the polar blackout during daylight hours seemed to be lower than that at night. In order to explain these results, the effect on the ionosphere of X-rays generated by the primary particles from the sun are discussed. The cause of magnetic bay disturbances is also considered. (Item 10.8-360, Met. Abs.).-.Author's abstract.

Mednikova, N.V., Ionosfernye vozmushcheniia osobogo tipa. (Particular types of ionospheric disturbances.) Aka. demiia Nauk, SSSR, Doklady, 5S(3):475-478, Jan. 21, 1948. 3 figs., ref. DLC.-Observations of an aurora on Feb. 16, 1947 carried out at the meteorological stations of the Institute of Terrestrial Magnetism near Moscow with the aid of a:1tomatic equipment with a frequency range from 2 to $16 \mathrm{MHz}$ led to the discovery of a particular type of ionospheric disturbance - an additional layer located above the normal F2 layer with critical frequencies much smaller than the ones of the F2 layer. The diffusivity of this layer increased with the growth of the disturbance but instead of rising as does the F2 layer it descended gradually. A subsequent careful examination of earlier records of ionospheric disturbance showed that from March 1946 to April 1947, 27 disturbances accompanied by appearances of this additional layer above F2 were record. e. I. It has been noted that this layer does not appear in all cases of high activity of auroras, but is always accompanied by auroras and at the time when the critical frequencies of F2 layer do not exceed $6 \mathrm{MHz}$. Photographs and graphs showing results of height and critical frequency measurement are included. (Item 6D-24, Met. Abs.)-.A. M. P.

Meek, J. H. , Ionospheric disturbances in Canada. Journal oí Geophysical Research, Wash., D. C., 57(2):177-190, June 1952. 11 figs. , 4 refs. DLC.-Analysis of variations of F region ionization and of abnormally high absorption of radio waves in northern latitudes indicates that disturbances appear first in one part of the auroral zone and then move round the earth with the sun for several days. The effect of a disturbance is enhanced and extend farther south near certain 
geographic latitudes. Diurnal and seasonal characteristics of the disturbances are described. It is suggested that dis. turbances are connected with similar geomagnetic disturbances and are due to a narrow stream of solar particles moving into the earth's path. .-Author's abstract.

C-197 Meek, J.H. (Defence Research Telecommunications Estab. lishment, Defence Research Board, Ottawa, Ontario, Canada), Correlation of magnetic, auroral, and ionospheric variations at Saskatoon. Joumal of Geophysical Research, 58(4):445. 436, Dec. 1953. 7 figs., 6 refs. DLC..-An analysis has been made for the five-month period from Dec. 1951 to April 1952 of the variations at Saskatoon of the horizontal component $(\mathrm{H})$ of the earth's magnetic field, the position in the sky and intensity of auroral light, and of critical frequencies and heights of the ionospheric reflecting regions. There is a relationship between the maximum elevation above the northern horizon of auroral light and the maximum amplitude of varia. tion of $\mathrm{H}$. Some types of sporadic $\mathrm{E}$ reflecting layers appear more frequently during disturbances. Detailed analysis of magnetically disturbed nights shows that magnetic bays and certain other phenomena are correlated. An increase in the intensity of aurora is related to the rate of decrease of $\mathrm{H}$ in the bay. Radio wave absorption or weak reflections at levels below $100 \mathrm{~km}$ correspond to the periods when $\mathrm{H}$ is of the order of 500 gammas or more from its normal value. (For Pt. 2, see ref. C-198). (Item 5C-260, Met. Abs.)--Author's abstract.

C-198 Meek, J.H. (Physics Dept, Saskatchewan Univ., Saskatoon, Canada), Correlation of magnetic, auroral, and ionospheric variations at Saskatoon, Pt. 2. Joumal of Geophysical Research, Wash., D. C., 59(1):87-92, March 1954. fig., 8 refs. MH-BH--The relations between magnetic, auroral, and ionospheric observations are summarized with reference to the occurrence of positive and negative magnetic bays. Auroral light associated with positive bays occurs at a higher geomagnetic latitude than that associated with negative bays. The magnetic and auroral light variations are compared to MARTYN's theory of the aurora. If the latter is accepted, the conclusion is reached (1) that most auroras are caused by positively charged particles, and (2) that the conditions described for the early phase actually exist throughout most of a disturbance. (For Pt. 1, see ref. C-197). (Item 6.10238, Met. Abs.) --Author's abstract. 
C-199 Meek, J.H. and McNamara, A. G. , Magnetic disturbances, sporadic $E$, and radio echoes associated with the aurora. Canadian Joumal of Physics, Ottawa, 32(5):326-329, May 1954. fig. , 6 refs. DLC--A comparison of simultaneous data on the visible aurora, the earth's magnetic field variation, vertical and oblique ionosonde echoes, and very high frequency radar echoes has been made. Long range highfrequency and very high frequency radio echoes do not appear to correlate individually. Reflections are observed, however, on both frequency ranges coincident with the appearance of low elevation auroral arcs, which are associated with magnetic bays. -.Authors' abstract.

C.200 Meos, Johan and Olving, Sven, On the origin of radar echoes associated with auroral activity. Goteborg, Sweden. Chalmers Tekniska Högskola, Handlingar, No. 196, 1958. 20 p. 14 figs., table, 21 refs. DLC..-Until very recently it has been accepted that most of the VHF radio echoes obtained near the polar regions during geomagnetic storms were due to direct scatter from auroral displays. HARANG and LANDMARK (1954) (See ref. C-127), however, have published a paper where they explain the echoes as backscatter from land or sea via intense sporadic ionospheric layers in the $E$ region. An analysis of the echoes at $10 \mathrm{~m}$ wavelength, recorded at the Kiruna Radio Wave Propagation Observatory during the first quarter of 1953, indicates that the echoes cannot be disconnected from aurora borealis. It is concluded that the predominant mechanism is closely connected to ionized and often invisible auroral forms. (Item 9H-129, Met. Abs.)--From authors' summary.

C-201 Merritt, Ernest and Bostwick, William E., A visual method of observing the influence of atmospheric conditions on radio reception. National Academy of Sciences, Wash., D. C. , Proceedings, 14(11):884-888, Nov. 1928. DLC.-.The experiment described is that of a partial separation of the ground waves and the sky wave in radio communication. Two balanced coils were used ( $A$ and $B$ ). The $A$ coil mounted with its plane vertical and directed toward the sending station, the $B$ coil in vertical plane at right angles to this direction. The latter coil responds only to that component of the sky wave which is polarized with its electric vector horizontal and is not affected by the ground wave. Suitable amplification to one pair of plates of a cathode ray oscilloscope featuring respectively horizontal and vertical movements, combination of which results in a Lissajous figure on the screen. An example of the method as used during the presence of aurora is described. Further studies especially during sunset are contemplated. (item 6D-10, Met. Abs.)... W. N. 
C-202 Mitra, Sisir K. (Prof. of Physics, Calcutta Univ.), The upper atmosphere. 2nd ed. Calcutta, The Asiatic Society, (Preface 1952). 713 p. figs., tables, bibliog. p. 644.668, eqs. DLC, MH-BH.-.The first edition of this valuable work, published in 1947, contained 616 pages. About 100 pages of new material have been added, including the results of the latest research in almost every field covered by the 13 chapters. These additions include work on escape of atmospheric gases (Chap. I), atmospheric tides (Chap. II), radar studies of meteors (Chap. III), variations in ozone content with latitude and air masses (Chap. IV), dissociation of $\mathrm{N}_{2}$ (Chap. V), ionospheric sounding by radio waves, estimation of recombination coefficients, tides and traveling disturbances in the ionosphere (Chap. VI), Sq and L variations near the geomagnetic equator (Chap. VII, up-todate bands and lines in auroral spectrum (Chap. VIII), new theories of magnetic storms and aurora by ALFVEN and MARTYN (extension of Chapman-Ferraro theory) (Chap. IX), Russian work on zodiacal light and afterglow or airglow, height measurements of luminescent layers, hydrogen and sodium in the upper atmosphere, etc. (Chap. X), revised model of temperature distribution in upper atmosphere (GER SON) (Chap. XI), a new chapter on rocket exploration of upper atmosphere (V.2 and aerobee flights) (Chap. XII), and new unsolved problems of the upper atmosphere (Chap. XIII). Brief discussion of winds at high levels from indirect meth. ods (Chap. XIII). No attempt at listing all of the important subjects covered in this work would do justice to its com. prehensiveness. The amount of carefully prepared illustra. tive material - charts, graphs, tables, schematic diagrams, etc. - both borrowed and original, is amazing. In most cases the sources are carefully cited and a class biblio. graphy of nearly 1000 references is a further aid in locating source material on all fields of upper air research by physical methods. (Item 4.8-15, Met. Äbs.)--M. R.

Moorcroft, D. R., Models of auroral ionization, Pt. I: Auroral ionization models and their radio reflection charac. teristics. Canadian Joumal of Physics, Ottawa, 39(5):677. 695, May 1961. ๑ figs., 18 refs., 45 eqs. DLC...Although radio observations of aurora contain information about the nature of the reflecting ionization, the use of dissimilar models of auroral ionization has led different workers to widely differing conclusions. In this paper several general models of auroral ionization are developed. By considering the ionization as an assembly of individual scatters, it has been possible to include a unified treatment of both weak scattering and critical reflections. This treatment should 
provide a basis for resolving some of the difficulties in the interpretation of auroral radio observations. In a second paper the available experimental evidence is examined in the light of this theoretical treatment.--Author's abstract.

C.204 Moorcroft, D. R., Models of auroral ionization. Pt. 2: Applications to radio observations of aurora. Canadian Journal of Physics, Ottawa, 39(5):695-715, May 1961. 8 figs., 30 refs., 13 eqs. DLC..In this paper the available experimental evidence concerning radio reflections from aurora is examined in relation to the reflection characteristics of the models of auroral ionization discussed in $\mathrm{Pt}$. I. The existence of critical reflection from auroral ionization at frequencies between 30 and $50 \mathrm{Mc} / \mathrm{s}$ appears to be estab. lished. This implies electron densities as great as $3 \times 10^{13}$ electrons per $\mathrm{m}^{3}$. It is shown that the observations are consistent with a model consisting of irregularities of ioniza. tion elongated parallel to the earth's magnetic field in a ratio of between 5 and 10 times, and having sizes transverse to the field lines of the order of a few meters. Some of the observations require the irregularities to be distributed in size. It is clear that there is a need for more relevant measurements on radio reflections from aurora to specify the characteristics of the ionization more precisely... Author's abstract.

C-205 Moore, R.K. , A VHF propagation phenomenon associated with aurora. Journal of Geophysical Research, 56(1):97-106, March 1951. 3 figs. , 3 tables, 6 refs. ..-Discusses auroral propagation as observed by radio amateurs operating at 28 to $148 \mathrm{Mc}$. Findings include: lack of skip effect, extremely high fading rate and insignificant change in polarization. North directed antenna gave better results. Number of days and hours with auroral propagation at geomagnetic latitudes $<52^{\circ}$ to $>60^{\circ}$ for the periods 1931 to 1941 and 1946 1950 are shown in tables and graphs. ..W. N.

C.206 Moore, R.K. , Aurora and magnetic storms. QST, West Hartford, Conn., 35(6):14-19+, June 1951. 9 figs. , 13 refs. DLC--A general discussion of the nature of ionospheric interferences with radio communication, particularly on the VHF bands used by radio amateurs. .. W. N.

C.207 Moore, R. K. , Theory of radio scattering from the aurora. Institute of Radio Engineers, Proceedings, 40(6):747, June 1952. DLC--Abstract of a paper presented at the I. R. E. Meeting, Washington, D.C. , April 1952. Details of a theory, with mathematical appendix, that signals observed are scattered from the leading edges of rapidly advancing columns of 
ionization (of diameter $\sim 50 \mathrm{~cm}$ ) created by bundles of incoming, $1 \mathrm{MeV}$, auroral protons. The theory explains the observed fading spectra (akin to meteor "whistle") and the minimum range effect (no retums at ranges less than $400 \mathrm{~km}$ have been reported). The rate of advance of the ionized columns is of the same order of magnitude as that found by spectrographic observation. Calculations of electron densities are attempted but depend on the size of the columns. ... Author's abstract.

C.208 Moore, R. K. (Sandia Corp.), Theory of radio scattering from the aurora. Institute of Radio Engineers, Transactions on Antennas and Propagation, AP 3:217-230, Aug. 1952. DLC.-It is postulated that radio signals returned from the aurora may be scattered by columns of ionization created by incoming auroral protons. The scattering from each column may be treated in the same manner as the "whistles" from meteor ionization, but because of the large number of columns created in a short time the "whistles" blend into a fading spectrum. Experimental determination of the fading spectra of such signals leads to curves which correlate well with those calculated by the theory. The velocities indicated agree in order of magnitude with that found by Gartlein's spectrographic observations. The calculated distances at which radar echoes should be observed agree with experiment and bear out the observations that signals are not heard or seen from overhead auroras. Density calculations cannot be complete because columnar size is not known, but indications are that this size may possibly be of the order of $1^{1 / 2}$ meters in diameter. -.Author's abstract.

C.209 Murcray, W. B. and Pope, J.H. (both, Geophys. Inst. Univ. Alaska, College, Alaska), Radiation from protons of auroral energy in the vicinity of the earth. Journal of Geophysical Research, Wash., D. C., 65(11):3569-3574, Nov. 1960. 3 figs. , 10 refs., eq. DWB, DLC.-Some considerations regarding the way in which auroral protons might be expected to radiate as they approach the earth are discussed. The form of the frequency-time curves which might result at the earth's surface from the radiation by such particles are deduced by assuming a reasonable model of the upper ionosphere. It is concluded, at least so far as the frequency-time curves are concerned, that auroral protons are capable of producing the low-frequency electromagnetic phenomenon known as "Chorus". (Item 12B-122, Met. Abs.)--Authors" abstract. 
C-210 Nakata, Yoshiaki (Radio Res. Lab. Kokubunji), Auroral echoes in the ionograms obtained in the minauroral region. Japan. Science Council. Ionosphere Research Committee, Report of Ionosphere Research in Japan, 12(1):1-5, 1958. 6 figs. (incl. photos), 6 refs. DWB, DLC.-A report of the radio echoes believed to be from auroral ionization obtained at a minauroral region (Japan) 3 times since the beginning of IGY by a continuously operated newly designed ionosonde. The instrumental details of the ionosonde are presented. These auroral echoes occurred on the magnetically disturbed days of Sept. 13 and 21, 1957 and of Feb. 11, 1958. The details of these echoes are discussed and their photographic representations shown. In order to indicate the source of these echoes, the relation between the calculated slant range and ionospheric height is considered and graphically represented. In this regard, evidence obtained at Kokubunji indicates that the echoes from auroral ionization have been obtained in fact from scattering source located at $F$ layer heights. (Item 12A.169, Met. Abs.)... I. S.

C-211 Nichols, Benjamin (Geophys. Inst., Univ. of Alaska, College, Alaska), Drift motions of auroral ionization. Journal of Atmospheric and Terrestrial Physics, London, 11(3/4): 292-293, 1957. fig., 3 refs. DLC.-Drifts $400-900 \mathrm{~km} \mathrm{~N}$ of College, Alaska were measured in VHF ranges (106 and $41.15 \mathrm{Mc} / \mathrm{s}$ ) from transmitters located $40 \mathrm{~km}$ E of College. Movement of echoes from auroral ionization in the $E$ region are probably due more to the speed of electrons in the disturbance current system than to ionospheric winds. Recent i snization data would indicate a total current of $10^{5} \mathrm{~A} / 100$ $\mathrm{km}$ of lat, in accordance with currents calculated from magnetic observations by SILSBEE and VESTINE (1942) and others. Radar echoes, spread over a range of $100 \mathrm{~km}$ are also consistent with electron density, drift velocity and magnetic observations. (See ref. C-167) (Item 10.3-332, Met. Abs. ).-M. R.

C-212 Nichols, Benjamin, Auroral ionization and magnetic disturbances. Institute of Radio Engineers, N. Y., Proceedings, 47(2):245-254, Feb. 1959. 3 figs., 56 refs. DLC--An examination of radio studies of auroral ionization shows that an average ionization density of about $5 \times 10^{5}$ electrons per $\mathrm{cm}^{3}$ is sufficient to explain the normal radar echo. The magnetic disturbances produced by current systems in the ionosphere are closely related both to the ionization and the luminosity of the aurora. The magnetic variations are also associated with increases in the speeds of motion of the ionization. The increased ionization manifest in the aurora, together with its increased speed of motion, brings about the 
magnetic changes observed at the ground. The rapid speeds of auroral motions observed both visually and by radio means are at the upper end of a continuous curve of drift motions that increase with increasing magnetic disturbance. (Item 11. 8-96, Met. Abs.).-Author's abstract.

C.213 Nichols, B. (Cornell Univ., N. Y.), Evidence of elongated irregularities in the ionosphere. Journal of Geophysical Research, Wash., D. C., 64(12):2200-2202, Dec. 1959. 11 refs. DLC--Radio observations of backscatter from ionos. pheric irregularities under both auroral and nonauroral conditions indicate the presence of small-scale irregularities, elongated along the earth's magnetic field. These elongated irregularities have been found at heights from 80 to $300 \mathrm{~km}$. The most precise measurements available are related to echoes from auroral ionization at a height of about $100 \mathrm{~km}$. These indicate scales of tens of meters along the earth's magnetic field and tens of centimeters normal to the field. (Item IIF. 95, Met. Abs. ).-Author's abstract.

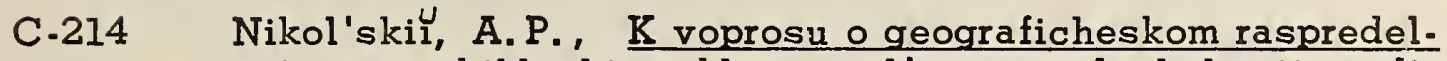
enii $\mathrm{v}$ vysokikh shirotakh anomal 'nogo pogloshcheniia radio. voln $\mathrm{v}$ ionosfere. (Geographical distribution in high latitudes of radio blackouts in the ionosphere.) Akademiia Nauk SSSR, Doklady, 112(4):628-631, Feb. 1957. 2 figs., table, 6 refs. Transl. into English available Special Libraries Association, Transl. Center (John Crerar Library, Chicago) as its R-1179 (6 p.). DLC.-Frequency, diurnal amplitude and phase of radio blackouts based upon analysis of foreign sources are discussed. The author uses the actual maximum instead of harmonic analysis. Evidence points toward a second maximum zone of blackouts near the poles in addition to the ring of maximum frequency in the zone of auroral activity. (Item 10.3-203, Met. Abs.)..A. A.

C-215 Nikolskil, A.P., O planetarnom raspredelenii magnitno ionosfernykh nozmushchenil i poliarnykh siianil. (World-wide distribution of magneto-ionospheric disturbance and aurora.) Akademiia Nauk SSSR, Doklady, 115(1):84-87, 1957. 3 figs., 9 refs. DLC. English transl. by E. R. Hope issued as Canada. Defence Research Board, Translation, T-266-R, Oct. 1957. DWB.-World-wide data on anomalous ionospheric absorption are interpreted according to Störmer's spiral model of solar corpuscular radiation distribution in the Arctic. Four zones of maximum incidence of corpuscular radiation are derived (extending into equatorial latitudes) and traced on a Northern Hemisphere chart. The existence of similar zones in the Southern Hemisphere is assumed and it is suggested that the intensification of magnetic activity at the equator 
might be explained by the overlapping of the two hemispheric distributions. (Item 12B-130, Met. Abs.).-G.T.

C.216 Norsk Institutt for Kosmisk Fysikk, The Auroral Observatory at Tromso" ( $\varphi=65^{\circ} 30^{\circ} .8 \mathrm{~N}, \lambda=18^{\circ} 50^{\prime} . \mathrm{E}$ G Gr. ). Observations 1952. Its Publikasjoner, No. 35, pub. 1954. 3! p. graphs, 4 figs., numerous tables. DWB..A brief des cription of the auroral and twilight sodium research (spectrograph observation) at Tromsö and Oslo, and ozone observations at Tromsö ( $S$ months) and Longyear, Spitsbergen ( 7 months) with a Dobson spectrophotometer. The Longyear observations were started in Sept. 1050 by S. T. I. LARSEN. Tile results of the first 2 years of observation there will be published in a separate paper. Following the 1-page discus. sion are tables of 03 values for each day of the observation period in 1952. Geomagnetic observations at Tromso" and Bear Island, radio echo observations and hourly ionospheric values of critical $E, F \circ E$, and $F 1, F 2$ and vertical height, storminess horizontal and vertical intensity and storminess values for each hour of the year are tabulated and shom in graphs. (Item 6.1-114, Met. Abs.)-.M. R.

C-217 Ohno, K. and Endo, K., The propagation of commercial s!lort waves through high latitudes. Japan. Radio Research Laboratories, Tolryo, Journal, $0(2): 13,1336$. Unchecired. .. Consider Nds to represent an index of the difficulty of commercial communications, if $s$ is the path length, and $N$ the a'roral frequency. --L. A. Manning.

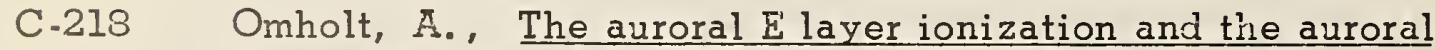
luminosity. Journal of $\overline{\mathrm{A}}$ tmospheric and Terrestrial Physics, 7(1/2):73-79, Fug. 1955. 2 figs., table, 10 refs. , 10 eqs. DWB--The processes causing increase in $E$ layer ionization during magnetic storms and auroras are discussed. It is shown that the recombination coefficient can be derived from simultaneous measurements of the photon emission irithin the first negative nitrogen band and the maxirnum electron density in the E region. Equipment and observations are des. cribed. The recombination coefficient during auroras is $>$ $10-7 \mathrm{~cm}^{3} \mathrm{sec}^{-1}$. In medium to strong auroras the mean electron density is $2-10 \times 10^{5} \mathrm{~cm}^{-3}$. VIF radio echoes require localized electron densities of about $1.4 \times 1.0 \mathrm{~cm}^{3}$, which tiough high may not be impossible. The variations of some purameters with height are discussed. (Item 7.1-230, Met. Abs. )--C.E.P.B. 
C.21G Onholt, A., Radio observations of aurorae. (In: Chicago Univ. Yerkes Observatory, Williams Bay, Wisc., Contract AF 1S(122)-480, Technical Report, No. 22 (Symposium on auroral observations during the IGY, Sept. 17-20, IS 3 ), pub. 1S50. p. 6.12. 18 refs..) DWB-.The possible altitudes and ionization processes which might account for radar and radio reflections from auroral streamers (associated with $E_{s}$ reflection) are discussed. The absorption of $1.20 \mathrm{Mc} / \mathrm{sec}: 7$ aves is supposed to take place at $30 \mathrm{krn}$, whereas the visible au. rora is $100 \mathrm{~km}$ or higher. Explanation is only tentative, radio echoes occur only with strong auroras low in NW and NE and usually with active rays. A strong fading rate and Doppler broadening indicate speeds of reflecting points up to 1000 $\mathrm{m} / \mathrm{sec}$. (Itern 11.4-120, Met. Abs.).-M. R.

C-220 Ortner, Johannes (Kiruna Geophysical Obs., Kiruna, Sweden), Around-the-world echoes observed on a transpolar transraission path. Journal of Geophysical Research, Wash., D.C., 64(12):2434-2467, Dec. 1SES. 3 figs., table, 4 refs. DLC.. Auroral influence on the $5200 \mathrm{~km}$ long communication path, 17. 500 and $24.025 \mathrm{Mc} / \mathrm{s}$ respectively, of the three backscatter sounders used, is discussed here. Multipath studies made Dec. 23, 1558 - Feb。24, 155S. The results are presented in table and graph. It was found that the field strength of the world travelling signal at times was not more attenuated as a direct propagated pulse. .-W. N.

C-221 Oscanyan, P.C., Jr., Radio phenomena recorded by the University of Michigan Greenland Expedition 1526. Institute of $R$ zdio Engineers, Proceedings, 15(5):425-430, May 1527. 5 figs. DLC--J. L. Reinhartz's statement "when a radio receiving station which plan to work on wavelengths of $50 \mathrm{~m}$ or be$\mathrm{L} \mathrm{N}$ is placed at the foot of a hill or mountain which is of a hoight greater than $17^{\circ}$ from the horizontal of the station, then signals will be screened off from the receiver" was so well verified that the radio station of the expedition had to move; since signals coming from north were completely blocked. An audibility graph disproves the popular belief that there is no static in the Arctic...W. N.

C-222 Oscanyan, Paul C. Jrn, Arctic auroral radio interference. QST, West Hartford, Conn, 13(12):10-20t, Dec. 1525. 5 figs., refs. DLC.-An account of auroral effects upon radio waves as observed by the author, in charge of the radio station of the Mt. Evans Observatory (Greenland $1200 \mathrm{f} . \mathrm{a}_{\mathrm{.s}}$.) of the Univ. of Michigan Expedition to Greenland 1527-1528. The receiving equipment described covered the frecuency range 15-30,000 KC. Apparently, this is the first report on direct observation of visible aurorae affecting radio wave 
propagation. The auroral effects are classified $A 1, B 1, B 2$ and $B 4$ according to interference on the various frequencies. It is noteworthy to quote: "What really offers the most interest is the fact that there seems to be a point which can be calculated. When the corona formation is nearest to $17^{\circ}$ (or more) above the horizon, and between the receiving and transmitting stations, the signal is most greatly influenced". Dire:tional movement of the radio disturbances are also consid. ered...W. N.

C.223 Owren, Leif; Leinbach, Harold and Nichols, B., Auroral absorption of radio waves transmitted via the ionosphere.

Alasia. Univ. Geophysical Institute, Contract DA-36-039-scS673S, Final Report (on Tasi A and B), March 1, 1555 to Feb. 25, 1050. 7ऽ p. 28 figs., 10 tables. DWB.-Give a detailed account of experimental data collected under Experi. ment Aurora. The monthly percentage of signal-in-time is tabulated for all frequencies and paths employed, and compared with East-West and South-North propagation at each freçuency. The seasonal variation in signal-in-time over short and long paths is shown in diagrams. There is a significant difference statistically in signal-in-time for the EastWest and North-South Paths for $12 \mathrm{mc}$ short paths. A study of the critical frequencies for $E$ and $F$ layers shows the difference in daytime variation of median signal strength between the years 1945-50 and 1SE4-5E due to changes in F lay. er ionization and D layer absorption in the course of a sunspot cycle. With a 12 mc radar, both direct backscatter and ground-scatter echoes were observed over a period of many months. Two types of echoes were recorded, one of which fades very rapidly. The data obtained about visual aurora at five stations in Alaska are analyzed. (Itern 12A-130, Met. A.j. ). A. H. K.

C-224 Parkinson, T.; Kirby, S. S. ; Arnold, P. N. and others, Bibliocraphy on radio vave phenomena and measurement of radio field intensity. Institute of Radio Engineers, Proceedings, 1:(6):1034-10E4, June 1@31. DLC--The bibliography contains 530 annotated entries classified under subject and related sibdivisions in chronological order: radiation, radio wave phenomena, fading, daily and seasonal variations, direction variations; meteorological, geophysical and cosmic effects; eclipses, reflection, refraction, diffraction, absorption, polarization, K-H layer, wave front angle, transmission formulas, atmospheric disturbances, strays, directional properties, etc. (Item SB-13, Met. Abs.)...W. N. 
C.225 Parthasarathy, R.; Basler, R.P. and de Witt, R.N., A new method for studying the auroral ionosphere using earth satellites. Institute of Radio Engineers, Proceedings, $47\left(\left(^{2}\right): 1650\right.$, Sept. 1959. DLC.-Simultaneous recordings are taken at two stations, $19 \mathrm{~km}$ apart, of the field strength of the $20 \mathrm{mc}$ transmissions from Sputnil III as it passes directly overhead. The results are used to study the structure of the absorption region. -.IRE, Proceedings, Abstract No. 4052.

C-226 Paton, James, The observation of aurora. International Council of Scientific Unions, Brussels, Eighth Report of the Commission for the Study of Solar and Terrestrial Relationships. Paris, 1G54. p. 157-157. 33 refs. DWB--Early maps of isochasms are based on inadeçuate data and close networks of observing stations have been recently established, supplemented by observations on transatlantic stratocruisers. A.ids to observing, analysis and classification are described; use of punched cards is suggested. Use of radio echoes is discussed. The paper ends with a list of outstanding problems. (Item 7.4-2SS, Met. Abs.).-C. E.P.B.

C.227 Penndorf, R. and Coroniti, Samuel C., Propagation of FF and VHF in the Arctic region. Institute of Radio Engineers, Transactions on Communication systems, CS-7(2):121-125, June 1C5S. 5 figs. , $\&$ refs. DLC.-Auroras can be used as reflectors or scatterers particularly in the afternoon and night, depending on local time. Critical analysis of all recordings by stations north of $50^{\circ}$ lat is reported on here. T:vo types of F2 propaga. tion are discussed along with the three sporadic E-types: 1. Thule, 2. auroral belts, and 3. mixed. ..W. N.

C.22E Pawsey, J.L., The question of radio emission by the ionosphere. Journal of Atmospheric and Terrestrial Physics, London, 15(1/2):51-53, Sept. 1959. 10 refs. DLC..The importance of non-thermal emission of radio waves of the ionosphere as a probable source of "radio noise from the aurora" in ionospheric and astronomical studies is examined. The themal emission from the $D$ region does not preclude occasional bursts nor intense emission from the $E$ layer. Intense noise from all over the sky reported on meter wavelengths, and other similar reports, until reliably reproduced, present clues rather than evidence. Brief increases of noise level on a frequency of $33 \mathrm{Mc} / \mathrm{s}$ coinciding with meteors might originate from a source other than the meteor. GALLET's report giving reasons for supposing the "dawn chorus" is generated in the outer reaches of the ionosphere is worthy of further examination. (Item 11.12-323, Met. Abs.).... T.

C.229 Peterson, A. M. and Leadabrand, R.L. (Radio Propagation Lab. , Stanford Univ. , Calif.), Long-range radio echoes from auroral ionization. Journal of Geophysical Research, 5c(2): 306-305, June 1S54. 2 figs. DLC.- 
Radio echoes were observed at 1500 to $4700 \mathrm{~km}$ from Stanford Univ. (43.75 $\mathrm{N}$ ) on frequencies of $0.42,12.80$ and $11.31 \mathrm{Mc}$ at greater heights than heretofore reported, and with intensities exceeding strongest $F$ layer propagated ground-scatter echoes occurring simultaneously. These echoes were first detected in Oct. 1 C52 during sporadic E layer studies. They are found in the F layer, not E layer zone as ras presumed. Discrete and diffuse echo records are reproduced. Several layers may be present at varying distances. Diurnal and sea. sonal frecuencies agree with auroral frequency data. (See ref. C-164). (Item 6D-357, Met. Abs。)--M。R.

C.230 Pickard, Greenleaf W., Aurora and low-frecuency radio recep. tion, July 7 to 8 , 1520. Terrestrial Magnetism and Atmospheric Electricity, 33(3):168, 1528. DLC.-.The aurora observed at Ne:uton Center, Mass. began at $20 \mathrm{~h} 15 \mathrm{~m}$ EST, July 7 as a bright arch in the northeast. An hour later an auroral crown developed near the zenith, with descending rays which at one time nearly filled the hemisphere, and which ivere at times strongly colored with pink and yellow -green. Broadcast reception from WGY at Schenectady, and KDKA at Pittsburgh was greatly depressed during the entire evening and did not rise appreciably above its normal lo daytime level. Although the Harvard Fistronomical Laboratory at Cambridge has not yet raduced its record of WBBM for this period, Stetson reports very low field values. My own record of field strength from siation WCI at Tuckerton, $N$ ew Jersey, operating at $10 \mathrm{KC}$, sho is a striking change from the normal diurnal curve for the past month, which had peaks at or near sunrise and sunset, with lo.y field during the night. On July $z$ the sunset peak "ras absent with high values during the greater part of the night, and insteacl of a poa'? at sunset a deep depression appears in the early noming record of July $S$, and a lo: and irregular field during the day. As there has been nothing like tis in the previous daily records of this station, it is assined that the change is associated with the aurora. -...Z. Z.

C.231 Pierce, J. $\AA_{0}$, Ionization by neteoric bombardment. Physical

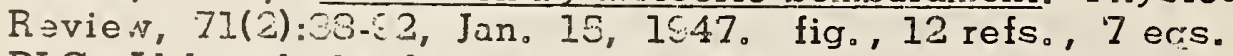
DLC.-Although the discussion centers on icaization by neteors there is a brief reference to patterns reflected by aurora borealis and sporadic E region ionization. ... E. Z.

C-232 Pogorelov, V.L., Radiolokatsionnye otrazheniia ot poliarny'h silanii. (Radar reflections from auroras.) Aleademiia Nauk SSSR, Izvestiia, Ser。Geofiz。, No。 8:1048-1051, 1C58. 4 figs, 6 refs, DLC. Transl, into English in the correspond. izg issue of the English language ed, of the Izvestiia, Bulletin of the Academy of Sciences of the USSR, Geophysics Ser. DWB.- 
In connection with the IGY program radar studies on the allrora are being carried out at Roshchino ( $\phi=60^{\circ} 12^{\prime} ; \lambda=$ $\left.25^{\circ} 34^{\prime} \mathrm{E} ; \Phi=53^{\circ} 35^{\prime} ; \Delta=110^{\circ} 47^{\prime} \mathrm{E}\right)$. The radar installation is described briefly. Two types of signals were observed on the screen of the electron tube indicator during an aurora; one type (a) was either stationary or moving very slowly and the other type (b) moved rapidly, both with increasing and diminishing distance. An examination of the distribution of both types of signals, at hourly intervals according to local time, shows that the time of maximum number of reflections and their maximum intensity coincide, and that weak signals were found to occur only at the time corresponding to that of maximim occurrence. With rare exceptions the variations in intensity with time of day is similar in both types of signals. The maximum reflection of both types of signals occurs along the azimuth at $0^{\circ}$ and $20^{\circ}$. It is possible that the small minimum along the azimuth near $10^{\circ}$ is a result of random deviation and it may disappear when more extensive material is analyzed. The mean position of azimuths, from which signals of different type issue, are often similar. Signals of type "b" are some. times either similar to or are mirror images of type "a" signals. The coincidence of a zimuths indicates that sometimes both types of radar reflections originate in similar regions with in. creased electron density. It is shown by means of a graph that the areas of reflection of signals are not located upon a narrow arc along a magnetic parallel, although fundamentally they are concentrated close to it. The arc-lile distribution coincides with the magnetic but not geomagnetic parallel. The measurement of the height of the radar reflection at Roshchino in 1957 indicates that the maximum number of reflections was at a height of $120 \mathrm{~km}$, i. e., primarily at the zone of distribution of the most intense regions of auroral illumination. When the magnetic field is replaced by a geomagnetic field another distribution is obtained. But the maximum number of reflections does not come from the azimuth coinciding with the plane of the geomagnetic meridian. (Item IIH-12I, Met. $\mathrm{Abs}$.).. I。L.D.

C.233 Presnell, R. I. ; Leadabrand, R.L.; Peterson, A. M. et al. (all, Stanford Res. Inst., Menlo Park, Calif.), VHF and UHF radar observations of the aurora at College, Alaska. Journal of Geophysical Research, Washa, D.C., 04(S):117@-11C0, Sept. 1SES. 13 figs., 6 tables; 14 refs. DLC.-During routine UHF auroral radar investigations an unusual daytime auroral effect has been discovered. It apparently occurs most frequently when: (1) the reflecting region is sunlit; (2) the atmosphere is undergoing its greatest change (early morning and late after. noon). There is a minimum of echo occurrence at noon when atmospheric conditions are stable. Daytime aurora is 
distributed over a larger region of space than the more commonly observed nighttime aurora. The nighttime and daytime echoes are labeled discrete and diffuse, respectively. They can be differentiated in several ways. Discrete echoes are identified by their relatively short duration, their occurrence only at night, and their orientation in the $E$ layer along a plane at right angles to radar beam; hence, the echo does not shift in range with change in elevation angle of the radar antenna. Diffuse echoes last longer, occur only during the day, and are apparently oriented in the E layer along a plane almost parallel to the surface of the earth; hence, the echo does shift in range when the radar-antenna elevation angle is changed. The primary effects of increasing the observation frequency are decreasing echo amplitudes and decreasing maximum off-perpendicular angle. The observed aspect sensitivity and the wave length dependence are interpreted in terms of the scattering approach of BOOKER.* Using the experimental UHF results, a model of the underdense ionos. phere has been developed consisting of irregularities which have dimensions of $0.1 \mathrm{~m}$ across and $3.5 \mathrm{~m}$ along the magnetic field lines. The echo results are compared with auroral zone effects and described, together with measurements of the frequency spectra (Doppler shift and spread) of an aurorally reflected continuous wave signal. (Item 12B-145, Met. Abst.) ('See ref. C-30)--Authors' abstract.

C-234 Rawer, Karl, Die Ionosphäre. Ihre Bedeutung für Geophysik und Radioverkehr. (The ionosphere. Its importance in geophysics and radio communication.) Groningen, P. Noordhoff, 1553. $17 \subseteq$ p. 67 figs., 143 refs., 68 eqs. DLC...A complete text on the use of radio in ionospheric research. Ch. I takes up methods of observation by echoes, spectroscopic methods for aurora and airglow, magnetic, meteor and luminous night clouds, and soundings; Ch. II gives results of observations by echo methods, magnetic data, composition, pressure, density and temperature. Ch. III discusses theories of ionos. pheric stratification (origin and disappearance of ionization and explanation of different layers. Ch. IV takes up normal and irregular changes of the ionosphere (D, E, F2, F, E2, G and $\mathrm{E}_{\mathrm{S}}$ and influence of magnetic storms, eclipses, polar summer and night, commencements). Ch. V discusses influence of ionosphere on propagation of radiowaves and foreCrsting propagation. (Item 6D-267, Met. Abs.)--M. R.

C.235 Reid, G. C. and Collins, C. (both, Defence Res. Board, Ottawa, Canada), Observations of abnormal VHF radio wave absorption at medium high latitudes. Joumal of Atmospheric and Terrestrial Physics, London, 14(1/2):63-81, April 1959. 7 figs., table, 23 refs., ec. DLC.. 
A study of cosmic noise absorption at a frequency of $30 \mathrm{Mc} / \mathrm{s}$ at Ottawa and Churchill has revealed the existence of two apparently distinct types of abnormal absorption event. One of these is predominantly a night-time phenomenon and is close. ly associated with auroral and geomagnetic disturbance. It is suggested that this absorption may be caused by an increase in electron collisional frequency at $E$ region heights rather than by a large increase in electron density at lover levels. The second type of absorption is confined to the auroral zone and is predominantly a daytime phenomenon, recurring for several days after a large solar flare. Evidence is presented to show that this absorption is due to an increase in ionization at very low levels in the ionosphere. The cosmic noise measurements are supported by evidence from a number of VIIF forivard scatter circuits in Canada, and this is used to obtain information about the geographical extent and frequency of occurrence of these abnormal absorption events. (Item 11E-133, Met. Abs.) -.Authors' abstract.

C-236 Rodewald, M. and Breitkreutz, Egon, Das grosse Nordlicht vom 21. Januar 19E7. (The great aurora borealis of Jan. 21, 1957.) Wetterlotse No. 113:43-54, Feb. 1857. 4 figs. DLC.-In $36^{\circ} 17^{\prime} \mathrm{N}, 6^{\circ} 46^{\prime} \mathrm{W}$ a red aurora penetrated by white rays. It was also seen in Portugal, Greece, Austria, Germany, etc. In $S$ iveden strong magnetic disturbances caused traffic interruptions. Radio signals were inaudible. An aurora of Nov. $\subseteq, 1956$ in $33^{\circ} \mathrm{N} 60^{\circ} \mathrm{W}$ is also referred to. (Item 3.6-367, Met. Abs.).-C.E.P.B.

C.237 Rumi, G. C., Experiment Luxembourg. Alaska. Univ. Geophysical Institute, Contract AF 1S(004)-3830, Scientific Report, No. 2, May 1559. 32 p. 12 figs., 13 refs. DWB (M(051) A323ex).-Auroral disturbances and the absorption of radio waves in the ionosphere have been investigated. The study shows that the absorption region is in two parts. In one of these layers the effective electron collision frequency increases during disturbed periods at auroral latitudes. This is attributed to auroral activity. The report concludes that at these auroral latitudes an auroral storm can even improve communications. (Item 12A-202, Met. Abs.)...N. N.

C-238 Rybner, Jörgen and Ungstrup, Emil, L'influence de la zone d'aurores boréales sur les liaisons radioélectriques. (Effect of the auroral zone on radio communication.) Annales des Télécommunications, Paris, 12(5):172-173, May 1557. fig., 3 refs. DLC..-Studies of high frequency propagation between Denmark and western Greenland are reported. Considering all possible modes of propagation, it is found that the unusiually weak field intensities observed may be attributed to D layer absorption in the auroral zone. (Item C. 5-273, Met. Abs. )-.G. T. 
C.23S Schallerer, W., Erdströme in Telegraphenleitungen im Zusam. menhang mit der Nordlichterscheinung am 25. 1. 1C30. (Terres. t:-ial currents in telegraph wires in correlation : $v$ ith aurora bore:ll is January 1, 1536.) Telegraphen, Fernsprech, Funk und Fernsehtechnik, 27(2):63-60, Feb. IS30. DLC..Extremely heavy oscillations of currents were observed in the ground crables at the UT/WT station in Berlin and elsewhere in Germany. With a periodicity of $\mathrm{min} 1 \mathrm{sec}$, maximum 2 minutes, t'ie phenomenon lasted from 2010 to 2200 and was stronger in the north-south oriented cables than in the east-west ones. In Arendal, Norway, the earth currents were more distinct in the aerial cables than in the ground cables. ..W. N.

C. 240 Sohlobohm, J.C. ; Leadabrand, R. L. and Dyce, R.B. et al. (all, Stanford Res. Inst., Menlo Park, Calif.), High altitude 106. $1 \mathrm{Mc}$ radio echoes from auroral ionization detected at a geomagnetic latitude of $43^{\circ}$. Joumal of Geophysical Re. search, Wash., D. C., 64(S):1101-1100, Sept. 1C59. 11 figs., table, 10 refs. DLC..Auroral echoes have been de. tected using a radar at 106. 1 Mc located at $43^{\circ}$ geomagnetic latitude. The geometry of reflection for ionization aligned with the earth's magnetic field lines is such that, for a geo. mignetic latitude of $43^{\circ}$, reflection can occur as high as $300 \mathrm{~km}$. The results of these observations are presented, with an interpretation of the height of reflections and a discission of the advisability of malking low latitude auroral echo investigations. (Item 11.7.144, Met. Abs.).-Authors' abstract.

C.24l Ssaton, S. L. and Malich, C.W., Auroral research at College, Alaska, 1041-1044, in Researches of the Department of Terrestrial Magnetism. Washington, D.C., Vol. 12, Part II. of Carnegie Institution of Washington, Publication INo. 175, 1 47 . p. 373-307. 2 figs., 2 tables. DLC.Description of buildings, personnel, instruments and methods, difficulties, calibrations, accuracy, discussion of data. All complete nighttime radio fade-outs occurring after midnight were accompanied by high intensity zenith aurora. Cameras and accessories illustrated. Tables giving zenith auroral intensities in equivalent photometer values, and corresponding values of upper frequency limits of blanketing type sporadic E reflections, Oct. 1043-March 1944 for 15 minute intervals. (Item 1.7-134, Met. Abs.)...M. R.

C-242 Seed, T.J. (Univ, of Canterbury, Neiv Zealand), V.F.F. obSirvations on the aurora australis. Journal of Geophysical Research, Wash., D.C., 33(3):517.523, Sept. 1C56. 3 figs., 20 refs. DLC.-Investigation of the aurora australis at a radio freciuency of ${ }^{\prime} \mathrm{Mc} / \mathrm{s}$ is reported. A value for the range 
exponent in the radar equation has been determined and values of the reflection coefficient deduced. Mechanisms of auroral reflection of radio waves are discussed, and only those involving column models are found to be substantiated. Noise emission from auroral ionization has been observed and measured at $69 \mathrm{Mc} / \mathrm{s}$. (Item 10.5-342, Met. Abs.).. Author's abstract.

C.243 Seed, T.J. and Ellyett, C. D. (both, Canterbury Univ. College, Christchurch, New Zealand), Low latitude reflections from the aurora australis. Australian Journal of Physics, Melbourne, 11(1):125-125, March 1@58. 2 figs., table, 5 refs. DLC.-A search for auroral reflections within a radar (or pulse radio transmitter) was begun in March $1 \subseteq 57$ at the Canterbury Univ. College Radio Observatory, $14 \mathrm{mi}$ from Christchurch, New Zealand (lat $43.6^{\circ} \mathrm{S}$, long. $172.6^{\circ} \mathrm{E}$ and $47.8^{\circ} \mathrm{S}$ geomagnetic lat). Echoes were obtained at Christchurch on March 10, 1557 for $3 \mathrm{hrs}(13-16 \mathrm{~h})$ at $325.500 \mathrm{~km}$ range and 116-105 $\mathrm{km}$ height. The aurora was observed visually over S. New Zealand, Campbell Island and Australia earlier, and over all of New Zealand from $13-14 \mathrm{~h}$, and in Aus tralia $16-1630 \mathrm{~h}$. Another radar display was observed on April 10. It is correlated with visual and magnetic data on a graph and vras calculated to come to within a distance of 250 $\mathrm{km}$ and $93 \mathrm{~km}$ height. The beginning of the radar return coincided with a steep gradient on the magnetogram. A total of 10 auroras were thus observed in 61 days (April 10-June 10) and the data are tabulated. (Item 10.2-341, Met. Abs.).. M. R.

C.244 Sen'ko, P. K. , Geofizicheskie issledovaniia v Antarktide. (Geophysical explorations in Antarctica.) Priroda, Moscow, 7:59-62, July 1958. 2 figs. DLC.-Some of the results of seismological, terrestrial magnetic and ionospheric observa. tions carried out in 1055 at Mirny, Antarctica, are summarized. The seismographs were capable of recording elastic oscillation of the ground with periods of $1-10 \mathrm{sec}$. In the first seven months some 200 earthquakes were recorded but only in about $1 / 5$ could the coordinates of the epicenters be established and for only half of these could the epicenter distances be computed. The centers of the earthquakes were in the southwest Pacific. The relationship between microseisms and synoptic conditions was investigated. In the winter-spring period, when the surface of the sea was covered with ice for hundreds of kilometers, microseisms with oscil. lation periods of $6.10 \mathrm{sec}$ were observed; in summer-fall, when ice fields are close to the shore, the microseisms have a smaller period but with an amplitude up to $7 \mu$. Short wave communication between Mirny and Moscon was best at 
night when radio waves were propagated along the shortest distance $(15,000 \mathrm{~km})$; in the afternoon a more intense signal passed through the regions of the South and North Poles $(25,000 \mathrm{~km})$. Owing to the absorption effect of the thick ice layer, radar communications in the heart of Intarctica could be carried on with medium length waves for short distances. The absolute and variable values of the elements of the earth's magnetic field were measured along the line MirnyPioneerskaia. At Mirny the magnetic field disturbance showe:d a maximum at noon or in the afternoon hours of mean local time. During the winter (May-Aug.) there was a second nocturnal maximum disturbance near local midnight in addition to daily maximum disturbance. In summer (Nov. -Jan.) there was a general increase in magnetic disturbances associated with an increased intensity of diurnal disturbances. At Mirny the aurora was observed primarily in the northern part of the sky. The magnetic variations as shown by the curve of the vertical component displayed considerable local differences within short distances ( 10 and $13 \mathrm{~km}$ ). The cause of the local varia. tions is attributed to an electrical current of abnomally large density flowing along the sea coast. The instrumentation and aims of studies on cosmic rays and auroras of the second continental expedition in 1557 are outlined. (Item 11.7.34, Met. Abs.)...I.L. D.

C.245 Shapley, Ellan H., Clues to ionospheric conditions in the southern auroral zone. Imerican Geophysical Union, Geo. physical Monograph, No. 1:03.60, 105. 4 figs,, ref. DLC.. While there is a considerable amount of experience with short wave radio communication via the ionosphere from the many Antarctic expeditions beginning in $1911-12$, quantitative determinations of ionospheric parameters exist for only a few places and for relatively short periods of time. From syeep frecuency vertical sounding observations at these fer points on tie Ant. arctic Continent and on surrounding islands, there is evidence of at least a rough north-south correspondence of $F$ region critical frequencies. The operation of a rather dense net ror? of stations during IGY will be needed for any real description or understanding of these phenomena. The objectives of a number of analyses with lata from the IGY period are mentioned. (Item 8.4-336, Met. Abs.)..-Author's abstract。

C.246 Shapley, Allan H., The ionosphere IGY programme in the Arctic. International Geophysical Year, 1057/155S, Conite Special, Bulletin d'Information, No.7:60-62, 1050. DWB.. Ionosphere program is closely linked thith the auroral and geo. magnetic and even the cosmic ray programs. Behavior of the ionosphere in auroral regions and features at or near the ITorth Pole during polar day and polar night will be investigated. 
Hemispheric symmetry will be studied by comparing Arctic and Antarctic data. Vertical soundings, studies of absorption, drifts, atmospheric noise ( $E$ stations in Arctic) and sferics (1 station in Spitzbergen), whistlers, and other phases of Arctic ionospheric program are discussed. (Item 10.3-30, Met. Abs. )--N. N.

C-247 Singer, S. Fred (Dept. of Physics, Maryland Univ.), 巫 new model of magnetic storms and aurorae. Anerican Geophysical Union, Transactions, 38(2):175-160, April 1057. It figs., 3 tables, 30 refs., eqs. DWB, DLC. Also issued as Maryland Univ. Physics Dept., Contract AF 1C(600)-1030, Technical Report No. 48 , July 1 CES. 20 p. 14 figs. , 3 tables in appendices. DWB-A new model for explaining S.C. 's (sudden commencements), magnetic storms, and the aurora is developed and presented in a lucid manner. Other molels are revieved (CIIAPMAN, FERRARO and ALFVENJ), observational evidence liscussed, and the pre-S. C. bays attributed to high velocity pirticles preceding the shock waves following $22-34$ hrs after a solar eruption. The shoc'z wave is retarded by the geomagnetic field, but accelerates as it enters the auroral zone and produces polar S.C.'s. The decrease in the storn is due to high velocity particles following up to $S$ hrs after the shock wave. The shock wave nay help accelerate auroral particles. (Item S. 4-276, Met. Abs.).-M. R.

C-210 Stein, Sidney (Radio Propagation Lab., Stanford, Univ., Stanford, Calif.), The role of $F$ layer tilts in detection of a:uroral ionization. Journal of Geophysical Research, Wash., D.C., $63(3): 361-50 \%$, June 1950. 6 figs., 15 refs. DLC.In important ionospheric mode for detection of porrerful longrange auroral reflections at Stanford depends upon appropriate tilts in the $F$ layer to the north. Auroral reflections received via this mode have been reported as originating from preval. ent strongly reflecting ionized structures at great heights, be$t$ reen $300 \mathrm{~km}$ and $1200 \mathrm{~km}$ above the surface of the earth. This interpretation is rejected as a plausible explanation of the radio data. An explicit distinction is made between these auroral reflections and newer classes of radio reflections observed at high and middle latitudes arising from field aligned ionization in the $\Sigma$ and $F$ regions. (Item 10. $5-210$, Met. Mbs.)... Author's abstract.

C-24S Stetson, Harlan True, Auroras, radio field strengths, and racent solar activity. Terrestrial Magnetism and $\bar{x}$ trospheric Electricity, $15(1): 77-96$, March 10.0. 2 tables, 11 refs. , 4 figs. DLC - $-A$ record of recent results of studies of changes $i_{1}$ conditions for radio transmission before and after the occur$r$ nces of auroral phenomena. Data of field strengths of 
WBBM's carrier wave at a frequency of $770 \mathrm{Kc}$ comprise over 100,000 measurements, covering ten minute intervals of night to night continuous readings. A table giving data from 15:30 through 1330 is included. -.E. Z.S.

C.250 Stetson, Harlan True, $\underline{z}$ note on occurrences of auroras, temperature changes and radio reception. Arnerican Meteorological Society, Bulletin, 23(1):21-23, Jan. 1\$42. DLC.Deals primarily with temperature relationships but refers to ridio field strength during aurora. It is noted that abnormally high field strengths persist in advance of the aurora. Subnormal field strengths persist for several days following the auroral date. -.E.Z.S.

C.251 Sťormer, Carl, Sur un echo d'ondes electromagnetiques courtes arrivant plusieurs secondes apres le signa emis; et son explication d'apres la theorie des aurores boreales. $(\mathrm{On}$ an echo of electromagnetic short waves arriving several seconds after emission of signals and its explanation by the theory of aurora borealis.) Academie des Sciences, Paris, Comptes Rendus, 187(15):811-312, Nov. 5, 1S23. DLC.An explanation of the phenomenon observed by Jörgen Fials at Bygdöy near Oslo, in the autumn of $1 \subseteq 27$ is attempted, based on simultaneous observations by Hals and the author, of echoes arriving at various intervals of 3 to 15 seconds and verified by van der Pol at the emitting station Philips Radio Eindhoven. Referring to author's research on aurora borealis in 1004 the echoes could have been originated in the cosmic space beyond the moon's orbit. Mathematical analysis proved, as early as 1S04, that electrical corpus. cles coming from very far (from the sun for example) could be deviated by an immense protrusion of the earth's magnetic field and unable to penetrate it, they could be reflected again under more favorable conditions and being still strong enough to cross the atmosphere they appear as echoes. Calculations ascertain the experimental results. -.O. T.

C.252 Störmer, Carl, Short wave echoes and aurora borealis. Nature, London, 122(3079):301, Nov. 3, 1023. DLC.-Des. cribes the reception of delayed echoes and attributes the de. lay to solar stream of particles. The study of these delays may throw light on electric currents in space outside the e.rrth and on their connection with the aurora borealis and magnetic storms. --E. Z.S. 
C.253 Störmer, Carl, Kurzwellenechos, die mehrere Seikunden nach dem Hauptsignal eintreffen, und wie sie sich aus der Tizorie des Polarlichtes erklären lassen. (Short wave echoes arriving several seconds after the transmitted signal, and how they may be explained according to the theory on polar aurora.) Naturwissenschaften, Berlin, 17(33):643-651, Aug. 1925. 5 figs., 3 refs., eqs. DLC...An account of the auroral radio echoes first observed by Jörgen Hals, Oslo, when listening in on the short wave station PCJJ, Eindhoven, Holland. The observations were subsequently verified by the author, van der Pol, E.V. Appleton and others. The tentative explanation oi the phenomenon involves Birkeland's historical experiment with the magnetic sphere, -.W. N.

C.254 Störmer, Carl, Über die Probleme des Polarlichtes. (On the problem of auroras.) Ergebnisse der Kosmischen Physik, Leipzig, 1:1-86, 1531. 70 figs., refs., ecgs. DLC-.One of the important contributions to our knowledge on auroras. A series of photographs shows different auroral forms. Photo. graphic equipment is briefly described. Determination of auroral heights shows frequency maxima for 101 and $106 \mathrm{~km}$, explained by atmospheric tides. A lengthy discussion is devoted to the phenomenon of auroral rays illuminated by the s:In, and to other unusual forms. Problems connected with the auroral spectrum are only briefly mentioned, but a detailed summary is given of author's mathematical theory of auroras, based on the equation of motion for charged corpus. cles in the magnetic field of an elementary magnet, showing that there are certain parts of the space around the magnetic e.arth (the ring form "torus space"), prohibited for electrical corpuscles. An experimental verification was found by the discovery of echoes of radio waves, reflected from the border surfaces of the "torus space", which had been already produced in a laboratory experiment by BIRKEL AND (1C01). The picture gets more complicated if gravitational forces are also considered in the theory. (Item 4J-70, Met. Abs.)...A. A.

C.255 S:örmer, Carl, The polar aurora. Oxford, Clarendon Press, 1555. 403 p. 213 figs. (incl. photos), numerous refs. throughout, tables, additional refs. p. 400-403. (International Monographs on Radio, ed. by Sir Edward Appleton and R. L. Smith-Rose). DLC (AC@71. S77). Review in Royal Met. Soc., Quarterly Journal, $82(3 E 1): 11 E$, Jan. IS50. .-This is the first substantial textbook on the aurora to be published in the English language by the man who has become ; for his life's work of photographing the aurora and studying iss nature. The book could y ell have consisted entirely of tize author's own findings which resulted mainly from over 50 years of study of parallactic photographs which he himself 
made by a method which he devised and many others have adopted. However, there is an abundance of reference to work of others in this field, as can be seen in the numerous foot-references and the added references to recent literature not cited in the text. The book is divided into two major parts which deal with: I. the descriptive aspects and II. the purely physical (atomic or molecular) and theoretical aspects. The 32 chapters cover, among other subjects: Pt. 1: 1) forms, 2) geographical description and periodicity, 3) methods of observation and photography, 4) height determinations by visual and 5) by photographic methods, 6) measurements near the a:iroral belt and 7) outside the auroral belt, 8) measurements and observations in S. Norway $1611-52$, S) sunlit auroras, 10) intensity, 11) color, 12) auroral spectra, 13) spectra of sunlit aurora, 14) radio waves $(10 \mathrm{~cm})$ from aurora, and brief review of notable work Forsyth, Petrie and Currie), 15) corpiscular currents outside atmosphere, 16) radio echoes from aurora observed at Jodrell Bank, Saskatoon, Canada; Kiruna, S reden and from Kjeller and Tromsö, Norway, 17) Doppler studies of corpuscular stream, 18) absorption of corpuscular rays by atmosphere, 19) emission of corpuscular rays from s:In. Pt. II: 1) differential equations, 2) results from study of differential equations, 3) families of orbits, 4) numerical integration, E) intuitive method of control, G) results of numexical integration (ALFVEN's method), 7) application of theory to laboratory experiments, 8) application of theory to polar aurora, ) limitations of theory, 10) approach of theory to actual conditions, 11) other theories (CHAPMAN-FERRARO, MARTYN and ALFVEN), 12) other applications, allowed and forbidden regions, solar, magnetic and coronal relations, etc. $A$ good author and subject index and scores of excellent original photographs are included. This is a most satisfying book - one that has been awaited 25 years. (Item 11A-1S, Met. Abs. ).-M. R.

C.256 Stoffregen, W. (Uppsala Ionos. Obs., Res. Inst. of Natl. Defence), Radio reflections on low frequencies from 75.00 kin height during intense aurora activity. Journal of Atmos. pheric and Terrestrial Physics, London, 13(1/2):167-100, Dec. 1950. 2 figs., 4 refs. DLC.-Observations with a new type o: ionosphere recorder constructed at the Uppsala Ionospheric Observatory and installed at Uppsala and at the new Ionos. pheric station at Lycksele $\left(64^{\circ} 37^{\prime} \mathrm{N}\right.$ and $\left.10^{\circ} 45^{\prime} \mathrm{E}\right)$ are presente.t. The occurrence of low frequency reflections ND from about $30 \mathrm{~km}$ level during strong auroral activity is illustrated on a diagram. Photos of ionosphere records show aurora $E$ reflections as well as reflections from about $30 \mathrm{~km}$ level on frequencies down to $0.33 \mathrm{Mc} / \mathrm{s}$. (Item 12A.232, Met. Abs.) -. $\mathrm{T}$. 
C-257 Stoyko, Nicolas and Jouaust, Raymond, Sur la propagation des ondes radioélectrigues courtes dans la région des aurores polaires. (On the propagation of radio electric short waves in t.he region of polar auroras.) Académie des Sciences, Paris, Comptes Rendus, 201(2):133-134, July 8, 1S35. DLC-.An analysis of measurements at Paris Observatory of short vave signals of a length of $37.08 \mathrm{~m}$ emitted from the station Honololu is presented on the verification of the hypothesis vhethe: anomalies observed on short wave propagation received at Tokyo from Washington were due to interference of auroral activity of the great circle between them. The signals receiv. et at Paris cross Greenland due to the magnetic north pole. Nevertheless they were observed as being of the same length as those of San Francisco which are a retransmission. Calculating a velocity of $270,200 \mathrm{~km} / \mathrm{s}$ for the speed of propagation between Honolulu and Paris, it is proved that the emissions of daytime related to those of nighttime received at Paris showed a systematic delay of 0.0518 . The direct wave transmission is completely explainable by the Sun. It is probable that the waves received at Paris did take the great circle Paris-Honolulu, which would be a so-called super-propagition. ... T. T。

C.258 Stranz, Dietrich, Eng begrenzte Ionisations: H"bhe wahrend einer Nordlichtstörung. (Isolated ionization clouds at height of $125 \mathrm{~km}$ during an auroral disturbance.) Archiv der elektrischen Ubertragung, $4: 213-213$, (ISEO). 3 figs., table. -From $22 \mathrm{~h}$ on Sept. 15, 1948 to $1 \mathrm{~h}$ on 10 th at Göteborg $3 \mathrm{MHz}$ waves showed isolated ionization clouds beginning at $200 \mathrm{~km}$ and descending to $125 \mathrm{~km}$. Attributed to a bundle of deflected corpuscular rays. There were pulsations of $3.5 \mathrm{sec}$ in intensity of echo. This observation raises a number of problems. The magnetic disturbance vector is analyzed. (Item 2.7.68, Met. Abs.)--C.E.P.B.

C-25S Sugiura, Masahisa; Tazima, Minoru and Nagata, Takesi, Anomalous ionization in the upper atmosphere over the auroral zone during magnetic storrs. Japan, Science Council. Ionosphere Research Committee, Report of Ionosphere and Space Research in Japan, Vol. 6, 1952. Unchecked...The depth of penetration and the rate of ionization of protons of extra ter. restrial origin were determined on the basis of the model atmosphere proposed by N.C. Gerson. It vas found that the energy of protons, which play an important part during magnetic storms, is several hundreds $\mathrm{KeV}$, or $1 \times 10^{\ominus} \mathrm{cm} / \mathrm{sec}$ in velocity. The rate of ionization of protons of this energy has a pronounced maximum near the bottom edge of the range of penetration. The life time of the ionized state was found to be of the order of a fei minutes, ten seconds. The density of 
precipitating protons required to maintain the ionized state was roughly estimated at 1 proton $/ \mathrm{cm}^{3}$ or less, which can be considered as reasonable values. An explanation was propissed for the violent and rapid variations in magnetic field observed in and near the auroral zone.

C-260 Taylor, Hoyt A. and Young, L.C., Studies of high-freciuency radio wave propagation. Institute of Radio Engineers, Proceedings, 10(5):561-573, May 1528. 7 figs. , 6 tables, refs. DLC-Quantitative measurements of "round-the-world" echoes at 16,700 and $19,000 \mathrm{kcs}$ made at the Naval Research Laborat rry, Bellevue, Anacostia since $1 \subseteq 27$ are discussed. Tabulateid data from stations in the U.S.A. and Europe shoued $\mathrm{s}=$ crlled direct signals "which appear to violate the skip distance la $\mathrm{N}^{\prime}$. A possible explanation is given, however, : ith reluctance "to entertain the idea of an additional layer with extremely high electron concentration and of so great a height, namely $1,400 \mathrm{~km}$, which would be necessary to explain the puth retarlations on these nearby echoes by reflections and multiple reflections from such a layer"..-W. $N$.

C-261 Thayer, Roger E., Radar echoes from the aurora borealis. Cornell University, Electrical Engineering Dept., June 1 S52. Thesis. Unchecked.

C.262 Thayer, Roger E., Auroral effects on television. Institute of Radio Engineers, Proceedings, 41(1):161, Jan. 1553. 3 f:gs., 3 refs。 DLC--During aurora displays, horizontal, indistinct, black bands appear on television screens in Ithaca, N. Y. They have been noticed on TV channels up through No. G(32.c8 $\mathrm{mc} / \mathrm{s})$. These appear on channels carrying no picture signals as well as those that do. Antenna rotation indicates that these disturbances are coming from the auroral zone.

C.253 Tomas, L. H., Short wave echoes and the aurora borealis. Nature, London, 123(30s2):166, Feb. 2, 1929. DLC-.The explanations for delayed echoes given by APPLETON and VAN DER POL are briefly reviewed. The suggested explanation would seem to be untenable unless it is assumed that $\vartheta$ is much longer than believed. If $v$ were 30 times as large the minimum reduction for a 10 sec delay would be to $e^{-4.0}$ of its initial value。.-.E.Z.S.

C-264 Tilton, Edward P., On the very highs。 QST, West Hartford, Cunn., 2E(3):41-43+, 1044. DLC-Auroral effects on radio iveves at $25,43-50,58$ and $112 \mathrm{Mc}$ are discussed in relation t $\supset$ radio amateur communication. .- W. N. 
C-265 Tilton, Edward P., Long distance communication over northsouth paths on $50 \mathrm{Mc} / \mathrm{s}$. International Council of Scientific Uniors. Mixed Commission on Ionosphere, 2nd Meeting, Brussels, Sept. 1950. Proceedings p. 119-120, publ. 1951. ref. DWB--During sunspot cycle peak of $1 \$ 47-48$, radio amateurs made discoveries regarding $50-54 \mathrm{Mc} / \mathrm{s}$ communication from Europe to South Africa and from North America to South America. Daily records made in Feb., March, Oct. and Nov. 1949 and Feb. -March 1950, showed that no communication wis possible between U.S. A. north of $40^{\circ} \mathrm{N}$ and South America except on the day following a visible aurora or ionospheric dis. turbance. (Item 5C-167, Met. Abs.)...M. R.

C-266 Ulrich, Franklin P., Auroral magnetic storms and difficulties in radio reception, $1 \subseteq 24-1 \subseteq 25$. Terrestrial Magnetism and Atmospheric Electricity, Wash., D. C. , 30(3):150-151, 1825. DLC--The results of observations at the Sit'ra Magnetic Observatory, U.S. Coast and Geodetic Survey are discussed. The appearance of aurora was reported ten times from radio stations during the reception of messages. On these ten occasions difficulty in reception was experienced only three times, once when the aurora was bright, once when the aurora was faint and once when the aurora was brilliant. On the seven occasions when no difficulty was experienced the aurora was faint on two occasions, bright on three occasions, and brilliant on two occasions. From the observations for 1523-1 24 no definite conclusion can be drawn as to difficulty of radio reception during aurora. The evidence from the 1C24. 1925 observations would indicate that the aurora does not in t.ie majority of cases cause any static. However, it is stated that a great number of observations should be made before any conclusion can be attempted. --E.Z. S.

C-267 Uirich, Franklin P., Äuroral observations, radio reception and magnetic conditions at the Sitka Magnetic Observatory, A xgust 1527 to June 1520. Terrestrial Magnetism and Atmos. pheric Electricity, Wash., D. C., 33(3):102-164, 1920. table. DLC.-This report is a continuation of the investigation begun in 1523, of the relation between aurora and the earth's magnetic field and of the effect of the aurora and magnetic conditions upon radio reception. The effects of aurora on radio reception are noted. Aurora was reported 58 times as follows: 31 faint; 21 bright; 3 brilliant. On the 31 times that faint aurora was reported reception was poor 3 times, fair 10 times, and good $1 E$ times. On the 21 times that bright aurora was reported radio reception was none one time, poor 2 times, fair 6 times, and good 12 times. Three brilliant auroras oc. curred and at those times reception was poor, fair and good once each. The observations for this year seem to indicate 
that good reception is very much more apt to occur than poor reception during a bright or faint aurora. In former years the results show that during a faint or bright aurora there was difficulty in radio reception about the same number of times as there were no difficulties. In former years the results were based on reports of cables and radio combined, while this year only reports of radio were considered. These results show that aurora causes poor reception in cable transmission, while in radio reception no difficulties are experienced in the inajority of cases. The relation between earth's magnetic field and $r$ dio reception is also discussed..-E. Z. S.

C.268 Ulrich, Franklin P., Auroral observations, radio reception, and magnetic conditions at the Sitka Magnetic Observatory, July 102E-June 1020. Terrestrial Magnetism and Atmospheric Electricity, Wash., D.C., 34(4):301-302, Dec. 1@2£. table. DLC--This report is a continuation of the reports begun in $1 \subseteq 23$ at Sitika Magnetic Observatory. It is noted that radio reception during the past year was much poorer than during the previous year. --E. Z.S.

C-260 Ulrich, Franklin P., Auroral observations and magnetic conditions at the Sitka Magnetic Observatory, Alaska, July 1520 to June 1530. Terrestrial Magnetism and Atmospheric Electricity, Wash., D.C., 36(3):23C-240, 1S31. DLC.-This paper is a continuation of the reports begun in $1 \subseteq 33$, of the investigation concerning the relation between aurora, the earth's magnetic field and radio reception. The investigation of the earth's magnetic field and its relation to radio reception was discontinued with the report last year because of the lack of proper sensitive instruments and because this investigation is being taken up elsevhere in a more detailed manner than was possible at this observatory. --E.Z.S.

C-270 Unwin, R。S. and Gadsden, M. (both, IGY Station, Dept of Sci. and Ind. Res., Ivarua Radio, Invercargill, New Zealand), Determination of auroral height by radar. Nature, London, 150(4500):1455-1470, Dec. 2S, 1SE7. 2 figs., 3 refs. DWB, DLC.-Auroral radio echoes observed by radar operating at 55 $\mathrm{Mc} / \mathrm{s}$ at Bluff in the South Island of New Zealand. A well defined aerial lobe pattern is obtained by interference of the direct and sea-reflected rays up to an angle of elevation of about 10. The polarization is horizontal resulting in deep minima between lobes. A range-time record of auroral echoes and a graph of observed heights of auroral echoing regions versus observed ranges are presented. The region producing the echoes occurs as an approximately horizontal sheet at an average height of $110 \mathrm{~km}$. (Item CII-101, Met. Abs.)--I.L.D. 
C.271 Unwin, R.S. (IGY Station of Dominion Physical Lab., Dept. of Sci. \& Indus. Res., New Zealand), The geometry of auroral ionization. Journal of Geophysical Research, Wash. , D. C. , 53(3):501-506, Sept. 1558. 5 figs., 7 refs. DLC.New evidence is presented which shows that VHF radio echoes from auroral ionization are reflections from aspect-sensitive columns aligned with the earth's magnetic field. Previous experimental data, interpreted in terms of a distribution of ionization along a line of magnetic latitude, are shown to be consistent with this hypothesis. (Item 10.5-343, Met. Abs.).Author's abstract.

C.272 Urwin, R. S. (D. P. L. Auroral Station, Dept. of Sci. \& Indus. Res., Awarua Radio, Invercargill, N.Z. ), Movement of auroral echoes and the magnetic disturbance current system. Nature, London, 133(4657):1044-1045, April 11, 1556. 2 figs., 11 refs. DWB--This preliminary report on radar investigations since 1558 at Invercargill is part of a research program of the Dominion Physical Laboratory of the New Zealand Dept. of Scientific and Industrial Research. The radar operating at 55 $\mathrm{Mc} / \mathrm{s}$ has its antenna beam orientated alternately on geographical bearings of $175^{\circ}$ for six min and $240^{\circ}$ for four min to avoid possible $210^{\circ}$ centered echoes. A histogram of all absolute directions observed in the period May-August features movement of echoes in terms of number of occurrences vs. direction, those with an $E$ or $W$ component in direction vs. universul time, and average velocities in the $800-1200 \mathrm{~km}$ range vs. Macquarie Island $\mathrm{K}$-index. The echoes came from a layer varying in thiciness up to $25 \mathrm{~km}$, and in tire altitude between 110 and $>120 \mathrm{~km}$. (Item 11.6-121, Met. Abs.).-W.N.

C-273 Unwin, R. S. (D.P.L. Auroral Station, Awarua Radio, Invercargill, N.Z.), Studies of the upper atmosphere from Invercargill, Nev Zealand. Pt. 1, Characteristics of auroral radar echoes at $55 \mathrm{Mc} / \mathrm{sec}$. Annales de Geophysique, Paris, 15(3): 377-3C4, July/Sept. 1SEC. (For Pts. 2 and 3, see refs. C-108, 105). 13 figs., 2 tables, 13 refs. French and English summaries $p$. 377. DLC.-Observations with a $55 \mathrm{Mc} / \mathrm{sec}$ radar e juipment in the south of New Zealand are discussed. Echoes associated with magnetic activity are recognized as being similar to the auroral echoes that have been observed in the Northern Fiemisphere over the past ten years. They may be divided into four major types sith distinct differences in appearance and times of occurrence. The height and thic'zness of the region producing the echoes is significantly different for each type, though most originate from the $110-120 \mathrm{~km}$ level. Although recorded during the second half of 1557 at a time of high solar activity, the frequency of occurrence appears to be much greater than at comparable geomagnetic latitudes in the 
Northern Hemisphere, though diurnal variations are similar. Systematic motions of the ionized regions are indicated, with a pronounced northward component before local magnetic midnight, and southward after. (Item 11.6-116, Met. Abs.).Author's abstract.

C-274 Van der Pol, Balth, Short wave echoes and the aurora borealis. Nature, London, 122(3034):373-37S, Dec. 8, 1S20. DLC.Various explanations for the delay of short wave echoes are considered. The length of the delay may be governed by the gradient of the electron density. Our view is that the "group" is compressed and 'bottled' for some time in those regions shere the group velocity approaches zero. -.E. Z.S.

C-275 Vassy, Etienne (Prof. Faculty of Science, Paris), Physique de l'atmosphere, Tome I. (Physics of the atmosphere, Vol. 1.) Paris, Gauthier-Villars, 1056. Ch. 1, Les aurores polaires. (Ch. 1, Polar auroras.) pp. 61-167. 75 figs., $\subseteq$ tables, eqs. DiC (QC330. V3)--The chapter on auroras occupies a major portion of this excellent text, giving perhaps the most complete survey of existing knowledge in this field. The material is treated in five sections. In Sec. $A$, descriptive phenomena, such as auroral forms, brightness, color, distribution in space and time, etc., are discussed, and relations to solar activity and atmospheric tides are considered. Sec. B contains a detailed summary of the results of auroral spectroscopy. Sec. C deals with radio emission and reflection from auroras. Sec. D is a review of various auroral theories, namely, the theory of corpuscular radiation of atmospheric origin, theories of BIRKE. LAND, STORMER, DAUVILLIER, CIIAPMAN-FERRARO, ALFVEN, and others. Finally, in Sec. E, applications of results of auroral research to investigations of upper atmospheric com. position, temperature, density and pressure are described. The chapter is illustrated throughout with representative data obtained by various authors. (Item 12B.164, Met. Abs.).-G. T.

C-276 Vegard, Lars, Die Deutung der Nordlichterscheinungen und die Struktur der Ionosphäre. (Explanation of auroral phenomena and the structure of the ionosphere.) Ergebnisse der Exakten Naturwissenschaften, 17:229-231, 1@30. 10 figs., \& tables, $10 S$ refs., 14 eqs. DWB-.This is a major treatise on all phases of auroral physics and theory bringing the author's 25 years of iror up to date and summarizing critically over a hundred works by other authors. The research embraced by this reviev falls into two categories: 1) the form, geographic distribution, time variations, relation to magnetic storms, earth currents and cosmis phenomena outside the earth's sphere of influence. These problems are kept uppermost in the author's presentation and are integrated by a single theory; 2) the physics of the aurora 
as determined mainly from recent research on the spectra of the aurora, radio reflections from the ionosphere and the night airglow. The scores of auroral lines identified at the time of this work are tabulated and their meaning in terms of the com. position and ionization of the atmosphere are specified. Resulting theory as to origin of aurora is treated in detail. The bibliography covers the period 1056-1S37. (Item 5C-91, Met. Abs.).-.M. R.

C.277 Vegard, Lars, Vorgänge und Zustände in der Nordlichtregion. (Processes and conditions in the auroral region.) Geofysis'ee Publikasjoner, Oslo, 12(5), 1038. 23+ p. 4 figs., 4 tables, 2 plates, 34 refs., 5 eqs. DLC.. A fundamental monograph giving the solution, or attempted solution, to a large number of problems concerning the physical properties of the aurora and the chemistry and physics of the ionosphere, from evidence gained through analysis of numerous auroral spectrograms in connection with magnetic, sunspot and radio propagation data. $\mathbb{A}$ few of the main points treated are: 1$)$ the explanation of the types of the red (relative to green) bands (colors) at the lower and upper portions of rays, 2) the tempsrature (and density) distribution at $25.150 \mathrm{~km}$, and 3 ) the intensity distribution and changes therein (relative and absolute). Over 100 spectral bands were obtained by the author and his colleagues, and over 80 of these identified. NJew bands recently discovered are listed and discussed. (Item 5C-33, Met. Abs.).-.M. R.

C-270 Vilbig, F.; Beckmann, V. and Menzel, W. , Utber Vorgänge in der Ionosphäre, săhrend des Nordlichtausbruches am 25 Januar 1933 in mittleren Breiten (52) festgestellt wurden. (Ionospheric processes as established during the aurora of Jan. 25, $1 \subseteq 38$ in the middle latitudes $\left(52^{\circ}\right)$.) Telegraphen. Fernsprech-Funk und Fernseh.Technik, 27(3):73-8I, March 1038. 4 figs., table, 3 refs., eq. DLC..The results of echo racordings as obtained in Pieskow at Scharmutzelsee from 13. 00 Jan. 25 to OSOO Jan. 27, 1538 on $86 \mathrm{~m}$ wavelength are presented and discussed. It was found that aurora at this latitude has two different reflecting layers, one of which penetrated the F layer, reduced the ionization present, caused a powerful sudden increase of the magnetic disturbance follos: ed by penetration of the radiation into the $E$ layer with subsequent ionization. The short save reception at Station Beelitz was characterized by the nightly after effect propagated from tire pole. The latitudinal effect on fading of these waves, mainly propagated via the F layer, was distinct...W. N. 
C.279 Watkins, C.D. (Jodrell Bank, Univ, Manchester), The height and geometry of auroral radio echoes. Journal of Atmospheric and Terrestrial Physics, N. Y., IC(1):1-r, Sept. 1S50. 7 figs., IC refs. DLC--The geometry of auroral radio eckoes detected at Jodrell Bank with low sensitivity equipments (wave length $A m$ ) is discussed. It has been found that echoes are only obtained from regions where the line of sight is within $1^{\circ}$ of perpendicularity to the lines of force of the local magnetic field at a height of about $110 \mathrm{~km}$, with a spread in height of the echo ragions of not rnore than 5 tha This conclusion is in disagreement with the original interpretation of BULLOUGI and KAISER (ISS4) (See ref, C-43), who proposed that the range azimutis characteristics of the echoes arose from the alignment of the reflecting regions along geomagnetic latitudes. The ne: result agress well with other observations carried out at Jodrell Bank on a rave length of $e \mathrm{~m}$ and ith observations of the aurora australis carried out in Antarctica and New Zealand on shorter wave lengths. It is suggested that small differences in the location of the echo-regions arise from changes in the height of the regions and distortions of the lines of force during magnetic disturbances. (Item 12A-243, Met. Abs.).-. Author's abstract.

C-230 Watkins, C. D. (Nuffield Radio Astronomy Lab., Jodrell Bank, Manchester Univ.), The magnetic storm-time variation of radio star scintillations and auroral radio echoes. Joumal of Atmospileric and Terrestrial Physics, London, IC(3/4):235-252, Dec. 1030. 3 figs., 3 reis. DWB.. $x^{5}$ n examination of the observations made at Jodrell Bank luring IS50-1950 sho:ts that about c) percent of all the series of raclio echoes recorded were preceded by a sulden commencement. This suggests that echo activity is mainly related to $s_{0} c_{\text {a }}$ type magnetic storms. The storm-time variations of echo incidence and mean scintillation rate are graphically presented. The variation of echo incidence is also plotted for the periods Jan. 1C55. June 1957 and July 1CE7 - Dec. 1CES separately and combined, and the associ.zted features are discussed. --I. S.

C-231 Watzins, C. D. (Royal Radar Estab., Malvern, Worcs.), Temporal variations of auroral radio-echo activity in sub-auroral latitudes. Journal of Atmospheric and Terrestrial Physics, London, 20(2/3):140.143, March 1@ல1. 6 figs., 16 refs. DíC.Low power radio-echo equipments have been continuously op. erated for several years recording observations of auroral ionization. The results have been examined for the following temporal variations of activity: diumal, 27 day, seasonal, and Il year. The time delay bet:reen the occurrence of class 3 solar flares and subsequent echo activity has also been investigated. It is suggested that the bimodal diurnal distribution 
pr)ssibly arises from the condition of specular reflection which controls the detection of echoes and from the north-south mo. tions of visual auroral forms. The general features of the other periodicities are found to be little different from the well known ones of geomagnetic disturbance. ...Author's abstract.

C.282 Watkins, C. D. (Royal Radar Estab., Malvern, Worcs.), Auroral radio echoes and magnetic disturbances. Journal of Atmospheric and Terrestrial Physics, London, 20(2/3):131-139, March 1C61. 6 figs., 8 refs. DLC.-It is shown that the probability of detecting auroral radio echoes with a low sensitivity equipment at Jodrell Bank increases with the value of the magnstic $K$ index, being 100 percent for local $K$ indices of 8 and $\theta$ There is a close correlation between east-west moving echo regions crossing the meridian of Eskdalemuir, Scotland, and magnetic disturbances recorded there. I comparison of magnetograms obtained at Lerorick and Eskdalemuir (north and south of the echo regions respectively) show that, in general, during the occurrence of echoes, the current systems giving rise to the magnetic disturbance are situated south of Lerwick and north of Eskdalemuir and thus near the echo regions. $\mathbb{B}$ in. vestigation of the location of visual auroral forms during the occurrence of echoes shows that they also are mainly situated in this region. The results confirm the suggestion of BULLOUGH et al.* (1957) that magnetic disturbances are closely related to regions of ionization which move approximately along magnetic parallels of latitude and which can be detected with radio echo equipments. (* See ref. C-45)..-Autlior's abstract.

C.283 Wex, Nelson, A note on design consideration for a proposed auroral radar. Göteborg, Sweden. Chalmers Tekniska Högs kola, Fandlingar, No. 102, 1857. $16 \mathrm{p} .10$ refs., 3 eçs. DL.C.-Examines the uses of a range-gated pulsed Doppler radar with filters and narrow beam widths. To that effect, the pertinent results of auroral studies are summarized, followed by some objectives and recommendations involved in the radar design discussed in comparison with MC NAMARE's system. 픈ㄱor's system, based on the significance of the fine structu:e of auroral echoes, pursues the fine azimuthal resolution and wide scanning ability. The principle outlined fundamen. tally exploits tested techniques and appears practical... W. N.

C.2e4 Weiss, A. A. and Smith, J. W. (both, Dept. Phys. U. of Adelaide), Radar record of an aurora at Adelaide. Journal of A tmospheric and Terrestrial Physics, London, 12(2/3):217.218, 1050. 2 refs. DLC..The aurora of Sept. 13, 1557 was visible throughout Australia and : was observed from Adelaide. Facts gathered were similar to those observed by HARANG and LAND. MARK in 1554 (See ref. C-127). The auroral echoes vere first 
heard from 1655 to $1927 \mathrm{LT}$ and the aurora was first seen at the limiting slant range of $900 \mathrm{~km}$. The echoes varied considerably during the course of the aurora. They first appeared with small amplitude at maximum range. The amplitude then increased until the range reaches a minimum value and thereafter decreased again as the range increased. (Item 10.6-127, Met. Abs.) --N. N.

C.285 Wells, H. W., Polar radio disturbances during magnetic bays. Terrestrial Magnetism and Atmospheric Electricity, Wash., D.C. , 52(3):315-320, Sept. 1947. 4 figs., 4 refs. DLC.-A comparison of ionospheric and magnetic records at College, Alaska, shows that marked increases in ionospheric absorption, producing partial to complete radio blackouts, occur during magnetic bays. The period of the absorption is limited to the duration of the magnetic bay. Particle bombardment is suggested as the cause of polar region radio blackouts and sporadic $E$ ionization. The observations indicate that these ab. sorption effects occur simultaneously over wide areas of $K$ 500 miles diameter.

C.286 Whale, H.A., The effects of ionosphere irregularities and the auroral zone on the bearings of short wave radio signals. Journal of Atmospheric and Terrestrial Physics, London, 13. (3/4):258-270, 1959. 3 figs., 12 refs. -.The nature of the observed variations in the received bearing of the signals from short wave radio stations at various distances is discussed and the origins of some of the effects are suggested. The major part of the daily variation of bearing of stations up to about $15000 \mathrm{~km}$ distant arises from the refraction of the ray in the $F 1$ region when it is reflected from the $F 2$ region. A dis. cussion of the curving of the ray path by successive small changes of direction at each reflection point leads to the concept of an antipodal area replacing the geometrical antipodal point. The large changes in direction associated with the passage of a ray through the auroral regions suggest a method of plotting the shape of the absorbing parts of the auroral zone by observations at a place remote from this zone. A sample plot obtained by this method is presented. (Item 12.4-357, Met. Abs.)--Author's abstract.

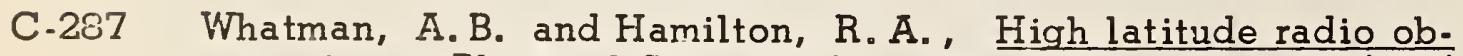
servations. Physical Society of London, Proceedings, 50(278): 217-232, March 1938. DLC.-The Oxford Univ. Arctic Expedition, $1 \subseteq 35-1 \subseteq 36$ to North-East Land $\left(80^{\circ} 23^{\prime} \mathrm{N}, 1 \mathrm{C}^{\circ} 31^{\prime}\right)$ observed no special conditions in the ionosphere during overhead auroras, nor any significant change of absorption. -.W. N. 
C-288 White, F. W. G. ; Skey, H. F. and Geddes, M., Radio fadeouts, auroras and magnetic storms. Nature, London, 142(3585):239, Aug. 13, 1938. 3 refs. DLC.- A brief letter sug. gesting that it would appear in high latitudes a radio fadeout may be due to ultraviolet radiation emitted during an erruption or may also be due to ionization by the particle radiation caus ing the auroras. Observations made in South Island, New Zealand Jan. 20.22 and Jan. 24.26, 1 537 , are discussed. The first of these periods began with solar activity and poor reflection from wireless waves from the ionosphere. On beginning observations of the reflection of waves from the F2 region on Jan. 20 no reflected wave could be observed. Magnetic conditions were moderately stormy and an aurora was observed over $\mathrm{New}$ Zealand. Similar events occurred during the second period. Ionospheric conditions in Christchurch appear to be similar to tizose observed at Tromsó by APPLETON (1937) during a magnetic storm and auroral display. It is found that "no echo" periods often occur in the morning following a night of magnetic and auroral activity. --E. Z.S.

C.289 Winckler, J. R. ; Peterson, L.; Hoffman, R. et al. (all, School of Physics, Univ. of Minnesota), Auroral storm of $\mathrm{Feb}$. 10-11, 1958. American Geophysical Union, Transactions, 39(6):1225. 1230, Dec. 1953. 3 figs. DWB, DLC.-During the night of Feb. 10-11, 1958, a very large auroral storm occurred. The storm and other related phenomena were especially well observed and reported, both visually and by a number of instrumental techniques. Balloon-borne instruments at Minneapolis detected two groups of strong X-ray bursts during the storm. These coincided with two large magnetic bays (increases or decreases in the intensity of magnetic-field components, re. presented by bay-like indentations on graphs showing varia. tions of magnetic intensity with time); with strong absorption of radio noise; and with the passage across the zenith of a very large amount of auroral luminosity. A cosmic ray de. crease also accompanied the storm. The University of Min. nesota workers believe that these terrestrial disturbances were associated with the earth's entry into a large cloud of solar gases that may have originated in a solar flare occurring about a day earlier. (Item 11. 1-314, Met. Abs.)--Auth. ors' abstract.

C.290 Yeh, K. C. (Univ. of Illinois) and Villard, O. G., Jr. (Stanford Univ.), Fading and attenuation of high-frequency radio waves propagated over long paths crossing the auroral, temperate and equatorial zones. Journal of Atmospheric and Terrestrial Physics, N. Y. 17(4):255-270, Feb. 1@60. 12 figs., table, 22 refs. DLC--This investigation is primarily concemed with the fading and attenuation of high frequency radio signals 
propagated over a long path crossing the auroral zone. The fading of high frequency signals propagated over non-auroral paths of comparable length has also been studied, and some new results are obtained. The principal fading and attenua. tion measurements on which these conclusions are based were carried out in Aug. 1957. For the auroral paths, there is no diurnal variation in fading speed except for a distinct minimum in time interval 1330-1900 PST, during which time the fading speed has little apparent dependence on magnetic activity along the path. In other time periods a positive correlation between magnetic activity and fading speed is found. It is suggested that the period of minimum fading speed is a consequence of the existence at that time of the kind of propagation mode made possible by ionospheric tilts. Attenuation over the long auroral-zone path is found to be associated with 'polar blackouts' as indicated by the absence of returned echo in vertical sounders located along the path. The percentage association varies with the location of the station relative to the path. This variation is consistent with the inferred propagation modes. It is found that during the hours 1330-1900 PST the attenuation cannot be attributed to the absorption that gives rise to blackouts as it can in the other hours. This is also explainable on the basis of the postulated tilt-mode propigation. Similar observations for temperate latitude and transequatorial paths of comparable length indicate that there is strong diumal variation in fading speed. Some plausible explanations are offered. (Item 11.10-188, Met. Abs.).-. Author's abstract.

C.2GI Zennek, J., Ionosphäre III. (Ionosphere III.) Ergebnisse der Exakten Naturwissenschaften, Berlin, 22:263-321, 1949. 47 figs., table, 125 refs., 23 eqs. DLC--This comprehensive discussion is devoted to the propagation of radio waves in the ionosphere including the influences on radio waves of the sun, meteors and aurorae. The discussion is arranged under the following main topics properly subdivided: (A) Experimental equipment; (B) Theories of electro-magnetic wave propagation in the ionosphere; (C) Normal state of ionization; (D) Abnormal E layer; and (E) Ionospheric disturbances. A good bib. li graphy containing 125 pertinent references arranged alphabetically by authors is included. .. W. N. 


\section{ANONYMOUS}

C.292 Auroral display and radio disturbance. Nature, London, 139(3512):318, Feb. 20, 1937. DLC..A brief note under the heading "News and Views". The occurrence of an aurora on Jan. 7 is confirmed by Mr. W. N. Craig of The Manse, Fort. rose, Ross-shire. Mr Craig, who was listening on the $14 \mathrm{Mc}$ amateurs band, found that reception from long distance sta. tions in South Africa and on the west coast of America, which was good at first, suddenly deteriorated so as to render the signals practically unintelligible by a very rapid flutter. $\mathrm{He}$ found that a conspicuous auroral display was in progress at the time. Magnetic traces recorded at the Greenwich magnetic station at Abinger show distinctive movements at the same time. -.E. Z.S.

C.293 Exploring the ionosphere. News of Norvay, Wash., D.C., 11(41):162, Nov. 18, 1954. DLC..Brief news report outlining the plans for Norwegian participation in the International Geo. physical Year, 1957-58. The Aurora Borealis Institute at Tromson will be the center of investigations mainly concerned with ionospheric soundings and auroral research. Other in. stitutes will participate and new facilities will be added to those available at Spitsbergen and Björnoya (Bear Island). (Item 6. 9.22 , Met. Abs.).-.G.T.

C.294 Poliamoe siianie 21-22 ianvaria 1957 goda. (Aurora of Jan. 21-22, 1957.) Priroda, Moscow, 46(12):33.85, Dec. 15E7. photo. DLC.-This is a review of information on the aurora described by a number of eye witnesses from the Moscow, Sumy (Ukraine), Yaroslavl and Murmansk Oblasts and mailed to the Murmansk branch of NIZMIR (Scientific Research Institute of Terrestrial Magnetism, Ionosphere and Radio-wave Propagation). The aurora was an outstanding geophysical phenomenon. Its origin was linked with an increase of solar activity. Its characteristic feature was the pre-eminently red color in more southern regions, which indicates its great height and its increased intensity. The aurora was accompanied by a very intensive ionospheric-magnetic disturbance and an interruption of radio com. munication on almost all lines. (Item 10.5-341, Met. Äbs.).-. A. M. P. 
C.2S5 Radio echoes from aurora. British Astronomical Association, Journal, 57(6):23S, Dec. 1@47. DLC--Brief note on echoes observed ith $46 \mathrm{Mc} / \mathrm{s}$ and $72 \mathrm{Mc} / \mathrm{s}$ equipment, A.ug. 15-15, at Jodrell Bank Experimental Station. The measured range of the echoes was $480 \mathrm{~km}$. The electron density apparently between $2.6 \mathrm{X} 10^{7}$ and $6.5 \mathrm{X} 10^{7}$ per c. c. is some 100 times greater than the normal local electron density during night. ..W.N.

C.2S6 Sizo:v model aurora. Science News Letter, Wash. , D.C. , 67(20):308, May 14, 1955. DLC.-Describes an experimental arrangement developed by WILLARD H. BENNETT of the Naval Research Laboratory in Washington. I small model earth is bombarded by electrons inside a vacuum tube. The existence of a ring current as first postulated by CHAPMAN, FERRARO and MARTYN is demonstrated in this experiment. It also sho:vs a simultaneous appearance of auroras in both hemis. pheres. (Item 7.8.271, Met. I.bs.).-G. T.

\section{ADDENDUM}

C.297 Chamberlain, Joseph W. (Yerkes Obs., Univ. of Chicago), Physics of the aurora and airglo: N. N. Y. , Academic Press, 1001. 704 p. Numerous figs., tables and eqs. Bibliog. p. ECC.670. (International Geophysics Series, ed. by J. Van Mieghem, Vol. 2) DLC, DWB (M21C443ph)..-Chapter 6 (p.217-243) of this new and exceedingly thorough revies of the entire field of the physics of the aurora, airglow and tisilight emissions, gives a two part summary of recently derived kno ledge of the radio aurora: 1) the observed characteristics: distinction between auroras and radio auroras; types of echoes, location of radio auroras, periodic variations, motions of ionization areas, polarizition, sensitivity and echo strength, and relationships with geomagnetic storms, fields, etc., and 2) theory of auroral reflections, geometry, critical and partial reflections from a large surface, scattering by small variations in ionization, and various mechanisms. The radio aurora (Collins and Forsyth 1359) designates ionization associated with auroras permitting radio reflections at VHF and $\operatorname{UHF}(30-3,000 \mathrm{Mc} / \mathrm{sec})$ bands. The aurora, which is visual (emitted), is an associate phenomenon with the same basic origin, but not always occurring simultaneously. Radio auroras go to greater heights and depths in the atmosphere than do auroras, and occur in day as well as night time, whereas certain forms of auroras can he seen at times and at aspects which do not correspond to radio auroral observation. 4 good number of references to literature on radio and radar auroral studies are cited. Other chapters give basic information up to 1960 on spectroscopy, photochemistry, etc. -.M.R. 


\section{AUTHOR INDEX}

Āarons, Jules C-1, 118

Agy, Vaughn C-2-6

Alfven, Hannes C.7

K.ppleton, E.V. C.3

A.rkhangel'skii, B. F. C-9

Arnold, P. N. C-224

Ispinall, A. C-10

Bailey, D. K. C-II

Bailey, V. F. C-12, 13

Barbier, Daniel C-14

Barlow, E. W. C-15

Basler, R.P. C-225

Bates, Howard F. C-16, 17

Beclmann, V. C.278

Bell, J. D. C-85

Bcllchambers, W. W. C-19

Belon, A. E. C-139

Berg, R.I. C-167

Besprozvannaia, A.S. C.79

Bhattacharyya, B. K. C-20

Bird, L. E. C-14.7

Birfel'd, Ia. G. C-2I, 22

Birkeland, Kristian C-24

Blevis, B.C. C-25, 25

Bontch-Brue:vitch, M. A. C -27

Booker, Henry G. C-28-32

Bost rick, William E. C-201

Bouchard, Jean C-33

Bowles, K.L. C-34-30

Braten, A. M. C-153

Breitkreutz, Egon C-236

Brown, Robert Ranbury C-40

Bruche, E. C -41

Budden, K. G. C -42

Bullough, K. C.43-45, 148

Burkhart, K. C-46

Burstall, T. C-163

Byrne, E. C. C.130
Cain, Joseph C. C -47

Cameron, E.A. C.26

Cartivright, D. G. C.92

Chamberlain, Joseph W. C-48, 49,297

Chapman, R.P. C-50

Chapman, Sydney C-5I-58

Chestnov, F.I. C.59

Chivers, H. J. A. C.60

Clegg, J.A. C.61, 176

Cohen, R. C-39

Collins, C. C-62,63, 235

Conference on Euroral Physics, July 23-25, 1951 C.64

Coroniti, Samuel C. C-227

Covington, $\mathbb{X} . \mathbb{E} . \mathrm{C}-65$

Cox, J. N. C.66

Currie, B.W. C-50, 67-69, 97O5, 154, 183, 184

Davidson, D. C-70

Davidson, T. W. C-45

Davies, K. C.66

De Kock, 可.C. C.7I

de Witt, R. R. C-225

Dieminger, Walter C-72-75

Dolphin, I.T. C.85, 166

Dostal, E. C-76

Dowden, R.L. C-77,78

Driatskii, V.M. C.79

Duncan, R. A. C.80

Dyce, Rolf Buchanan C-81-85, 167,240

Eastwood, E. C-86

Eckersley, T.L. C-5I, 87

Egan, R. D. C.88

Egeland, Alv C-89, 118

Ellis, G. R. A. C-80, 90-92

Ellyett, C. D. C-61, 243

Ellyett, G. E. C-176

Endo, K. C.217 
Fanselau, Gerhard C-93

Fendler, Ernst C-94

Ferrel, Perry, Jr. C.95

Fleischer, R. C-96

Forsyth, P. A. C-63, 67, 97-99,

$$
\text { 100-104 }
$$

France. Meteorologie Nationale

$\&$ Societe Meteorologique

de France C-105

Fricker, S.J. C-106

Fulton, B. J. C-107

Gadsden, M. C-100, 270

Gartlein, C. W. C-28

General, J. C-153

Gerson, N. C. C-110-114

Gilliland, T. R. C-155

Glass, D. G. C-152

Götz, Friedrich Wilhelm Paul C.115

Green, F. D. C-104

Greenhow, J. S. C.116

Groves, J. R. V. C.92

Gusev, P.I. C-117

Gustafsson, Georg C-118

Hagg, E.L. C.119, 120

Hakura, Yukio C-121, 122

Hamilton, R.A. C.287

Hanson, G. H. C-IIO

Harang, Leiv C-123-128

Harrison, D. P. C-129, 130

Harrison, V.A.W. C.13I

Hartz, T.R. C-132, 133

Hawkins, G.S. C-10, 134

Helbronner, Paul C-135

Hellgren, Gösta C-136

Helliwell, R.A. C-101

Hellman, Gustav C-137

Fienderson, J.P. C-138

Heppner, J. B. C-139

Heppner, James C-140

Herlofson, N. C-141

Hoffman, R. C-289

Hoffmeister, C. C-142

Hultqvist, Bengt, C-8S
Ingalls, R.P. C-106, 147

Institute of Radio Engineers C. 143

Isaev, Sergei Ivanovich C-144, 145

Israël, Hans C-146

Isted, G. A. C.86

James, J. C. C-147

Jouaust, Raymond C-257

Kaiser, T. R. C-43-45, 148-151

Kavadas, A. C-152, 178

Kenrick, G. W. C-153

Kim, J.S. C-154

Kirby, S. S. C-155, 224

Knecht, R.W. C-156

König, Hermann C-142

Krüger, K. C-157

Landmark, Björn C-127, 158

Lange-Hesse, Günther C.160

Larmor, Joseph C-16I

Lauter, E. A. C-162

Law, P.G. C-163

Leadabrand, R.L. C-164-167, $229,233,240$

Leinbach, Harold C-223

Leithaüser, G. C-168

Leonard, Robert S. C-169, 170

Lied, F. C-171

Lindquist, Rune $\mathbb{A}$. C-172, 173

Little, C. Gordon C-56, 174, 175

Lovell, A.C.B. C-40, 176

Lugeon, J. C-177

Lyon, G. F. C-178, 179

Mc Innes, B. C-180, 181

Mc Kinley, D. W. R. C-182

Mc Namara, A. G. C-183-188, 193

Maehlum, Bernt C-189

Mah, W. C-104

Major, G. C-190 
Malich, C. W. C.24I

Martin, L.H. C-191

Martvel', F.E. C-192

Massey, H. S. W. C-193

Matsushita, S. C-194

Mednikova, N.V. C-195

Meek, J. H. C-196-199

Menzel, W. C-278

Meos, Johan C-136, 200

Merritt, Ernest C-201

Millman, Peter M. C-182

Mitra, Sisir K. C.202

Moorcroft, D. R. C-203, 204

Moore, R. K. C-205-208

Muldrew, D. C-120

Murcray, W. B. C-209

Nagata, Takesi C.259

Nakata, Yoshiaki C.210

Neufeld, E.L. C-116

Nichols, Benjamin C-28, 211 213,223

Nikol'skii, A.P. C-214, 215

Norsk Institutt for Kosmisk

Fysikk C-216

Ochs, G. R. C-39

Ohno, K. C-217

Olving, Sven C-200

Omholt, A. C-218, 219

Ortner, Johannes C-89, 220

Oscanyan, Paul C. , Jr. C-221, 222

Otsuki, Toshiharu C.121

Orvren, Leif C.223

Pabo, N. V. C.9

Parkinson, T. C.224

Parthasarathy, R. C-225

Paton, James C-226

Pawsey, J.L. C-228

Penndorf, Rudolf C-115, 227

Peterson, A. M. C-88, 164, 166, 229,233

Peterson, L. C-289

Petrie, L. E. C-107
Petrie, W. C-97-99

Pickard, Greenleaf W. C.230

Pierce, J. A. C.231

Piggott, W. R. C-19

Plendl, H. C.73

Pogorelov, V.I. C.192, 232

Pope, J. H. C-209

Presnell, R. I. C-167, 233

Pushkov, N. V. C.145

Rawer, Karl C.234

Rayton, W. M. C-175

Reid, G. C. C-132, 235

Robertson, K.A. C-131

Rodewald, M. C-236

Roof, R. B. C.175

Rumi, G. C. C-237

Rybner, Jörgen C-233

Schallerer, W. C-239

Schlobohm, J. C. C-240

Seaton, S. L. C-241

Seed, T. J. C.242, 243

Sen'ko, P.K. C-244

Shapley, Allan H. C-245, 245

Singer, S. Fred C-247

Smith, J. W. C.284

Stein, Sidney C.248

Stetson, Harlan True C-249, 250

Stoffregen, Willi C-123, 125, 256

Stone, M.L. C-106

Störmer, Carl C-251-255

Stoyko, Nicolas C.257

Stranz, Dietrich C.258

Sugiura, Masahisa C-259

Takenoshita, Yugoro C-121, 122

Taylor, Hoyt A. C-260

Tazima, Minoru C.259

Thayer, Roger E. C.262

Thomas, L. H. C.263

Tilton, Edward D. C-264, 265

Tröim, J. C-128 
Ulrich, Franklin P. C.266250

Ungstrup, Emil C-238

Unwin, R.S. C-270-273

Van der Pol, Balth C.274

Vassy, Etienne C.275

Vawter, F.E. C.67, 99

Vegard, Lars C-276, 277

Vilbig, F. C.278

Vogan, E.L. C-102, 132

Wang, S.C. C.106

Warren, E. C-120

Watkins, C. D. C.45, 116, $129,279-232$

Wax, Nelson C-283

Weiss, A. A. C.284

Wells, H. W. C.60, 285

Whale, H. A. C-236

Whatman, A.B. C-237

White, F.W. G. C.283

Winckler, J. R. C-239

Yabroff, I. C-165

Yates, G. G. C-42

Yeh, K.C. C.290

Young, L. C. C-260

Zennek, J. C-291

ANONYMOUS

C-292-296 


\section{THE NATIONAL BUREAU OF STANDARDS}

The scope of activities of the National Bureau of Standards at its major laboratories in Mashington, 1).C., and Boulder, Colorado, is suggested in the following listing of the divisions and sections engaged in technical work. In general, each section carries out specialized research, development, and engineering in the field indicated by its title. A brief description of the activities, and of the resultant publications, appears on the inside of the front cover.

\section{WISIILNGTON, D.C.}

Electricity. Resistance and Reactance. Electrochemistry. Electrical lnstruments. Magnetic Measurements. Dielectrics. High Voltage.

Metrology. Photometry and Colorimetry. Refractometry. Photographic Research. Length. Engineering Metrology. Mass and Scale. Volumetry and Densimetry.

Heat. Temperature Physics. Heat Mleasurements. Cryogenic Physics. Equation of State. Statistical Physics. Radiation Physics. X-ray. Radioactivity. Radiation Theory. High Energy Radiation. Radiological Equipment. Nucleonic lnstrumentation. Neutron Physics.

Analytical and Inorganic Chemistry. Pure Substances. Spectrochemistry. Solution Chemistry. Standard Reference Materials. Applied Analytical Kesearch.

Mechanics. Sound. Pressure and Vacuum. Fluid Mechanics. Engineering Mechanics. Rheology. Combustion Controls.

Organic and Fibrous Materials. Rubber. Textiles. Paper. Leather. Testing and Specifications. Polymer Structure. Plastics. Dental Research.

Metallurgy. Thermal Metallurgy. Chemical Metallurgy. Mechanical Metallurgy. Corrosion. Metal Physics. Electrolysis and Metal Deposition.

Mineral Products. Engineering Ceramics. Glass. Refractories. Enameled Metals. Crystal Growth. Physical Properties. Constitution and Microstructure.

Building Research. Structural Engineering. Fire Research. Mechanical Systems. Organic Building Materials. Codes and Safety Standards. Heat Transfer. Inorganic Building Materials.

Applied Mathematics. Numerical Analysis. Computation. Statistical Engineering. Mathematical Physics. Operations Research.

Data Processing Systems. Components and Techniques. Computer Technology. Measurements Automation. Engineering Applications. Systems Analysis.

Atomic Phys ics. Spectroscopy. Infrared Spectroscopy. Solid State Physics. Electron Physics. Atomic Physics. Instrumentation. Engineering Electronics. Electron Devices. Electronic Instrumentation. Mechanical Instruments. Basic Instrumentation.

Physical Chemistry. Thermochemistry. Surface Chemistry. Organic Chemistry. Molecular Spectroscopy. Molecular Kinetics. Mass Spectrometry.

Office of Weights and Measures.

\section{BOULDER, COLO.}

Cryogenic Engineering. Cryogenic Equipment. Cryogenic Processes. Properties of Materials. Cryogenic Technical Services.

Ionosphere Research and Propagation. Low Frequency and Very Low Frequencv Research. lonosphere Research. Prediction Services. Sun-Earth Relationships. Field Engineering. Radio Warning Services. Vertical Soundings Research.

Rad1o Propagation Engıneering. Data Reduction Instrumentation. Radio Noise. Tropospheric Measurements. Tropospheric Analysis. Propagation-Terrain Effects. Radio-Meteorology. Lower Atmosphere Physics.

Radio Standards. High Frequency Electrical Standards. Radio Broadcast Service. Radio and Microwave Materials. Atomic Frequency and Time Interval Standards. Electronic Calibration Center. Millimeter-Wave Research. Vicrowave Circuit Standards.

Radio Systems. Applied Electromagnetic Theory. High Frequency and Very High Frequency Research. Modulation Research. Antenna Research. Navigation Systems.

Upper Atmosphere and Space Physics. Upper Atmosphere and Plasma Physics. Ionosphere and Exosphere Scatter. Airglow and Aurora. Ionospheric Radio Astronomy. 


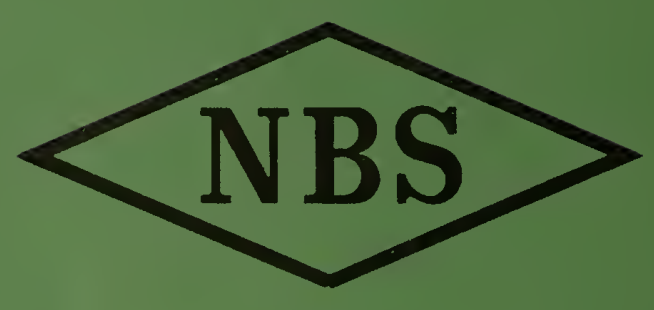

